OPEN ACCESS

Edited by:

Rayne Rouce,

Baylor College of Medicine,

United States

Reviewed by:

Premal Lulla,

Baylor College of Medicine,

United States

Zong Sheng Guo,

School of Medicine, University of

Pittsburgh, United States

*Correspondence:

Henry T. Marshal

henry_marshall@me.com

Specialty section:

This article was submitted to Cancer Immunity and Immunotherapy, a section of the journal Frontiers in Oncology

Received: 13 May 2018

Accepted: 24 July 2018

Published: 23 August 2018

Citation:

Marshall HT and Djamgoz MBA (2018) Immuno-Oncology: Emerging Targets and Combination Therapies.

Front. Oncol. 8:315

doi: 10.3389/fonc.2018.00315

\section{Immuno-Oncology: Emerging Targets and Combination Therapies}

\author{
Henry T. Marshall* and Mustafa B. A. Djamgoz \\ Neuroscience Solutions to Cancer Research Group, Department of Life Sciences, Imperial College London, London, \\ United Kingdom
}

Host immunity recognizes and eliminates most early tumor cells, yet immunological checkpoints, exemplified by CTLA-4, PD-1, and PD-L1, pose a significant obstacle to effective antitumor immune responses. T-lymphocyte co-inhibitory pathways influence intensity, inflammation and duration of antitumor immunity. However, tumors and their immunosuppressive microenvironments exploit them to evade immune destruction. Recent PD-1 checkpoint inhibitors yielded unprecedented efficacies and durable responses across advanced-stage melanoma, showcasing potential to replace conventional radiotherapy regimens. Neverthless, many clinical problems remain in terms of efficacy, patient-to-patient variability, and undesirable outcomes and side effects. In this review, we evaluate recent advances in the immuno-oncology field and discuss ways forward. First, we give an overview of current immunotherapy modalities, involving mainy single agents, including inhibitor monoclonal antibodies (mAbs) targeting T-cell checkpoints of PD-1 and CTLA-4. However, neoantigen recognition alone cannot eliminate tumors effectively in vivo given their inherent complex micro-environment, heterogeneous nature and stemness. Then, based mainly upon CTLA-4 and PD-1 checkpoint inhibitors as a "backbone," we cover a range of emerging ("second-generation") therapies incorporating other immunotherapies or non-immune based strategies in synergistic combination. These include targeted therapies such as tyrosine kinase inhibitors, co-stimulatory mAbs, bifunctional agents, epigenetic modulators (such as inhibitors of histone deacetylases or DNA methyltransferase), vaccines, adoptive-T-cell therapy, nanoparticles, oncolytic viruses, and even synthetic "gene circuits." A number of novel immunotherapy co-targets in pre-clinical development are also introduced. The latter include metabolic components, exosomes and ion channels. We discuss in some detail of the personalization of immunotherapy essential for ultimate maximization of clinical outcomes. Finally, we outline possible future technical and conceptual developments including realistic in vitro and in vivo models and inputs from physics, engineering, and artificial intelligence. We conclude that the breadth and quality of immunotherapeutic approaches and the types of cancers that can be treated will increase significantly in the foreseeable future.

Keywords: combination immunotherapy, tumor microenvironment, checkpoint blockade, personalized therapy, biomarkers 


\section{INTRODUCTION}

Non-surgical treatments of cancer (mainly conventional chemotherapy, targeted biological therapies, and radiotherapy) have not generated completely satisfactory results to date. The ongoing problems include low target selectivity, drug resistance, inability to effectively address metastatic disease and severe side effects. In contrast, immunotherapies that overall provoke host immunity to induce a systemic response against tumors currently offer much clinical promise. Although most malignant tumors

\footnotetext{
Abbreviations: ACT, adoptive cellular therapy; APC, antigen presenting cell; ARG, arginase; B-ALL, B-cell acute lymphoblastic leukemia; BBB, blood-brainbarrier; BiTE, Bi-specific T-cell engager; BTLA, B- and T-lymphocyte attenuator; CAF, cancer-associated fibroblast; CAR, chimeric antigen receptor; CCL2, CC motif chemokine ligand 2; $\mathrm{CD}$, cluster of differentiation; cdGMP, cyclic di-guanosine monophosphate; $\mathrm{CDK}$, cyclin-dependent kinase; $\mathrm{CML}$, chronic myeloid leukemia; COX2, cyclooxygenase 2; CRAC, calcium release activated channel; CRISPR, clustered regularly interspaced short palindromic repeats; CRS, cytokine release syndrome; CSC, cancer stem cell; CT, computed tomography; ctDNA, circulating DNA; CTL, cytotoxic T-lymphocyte; CTLA-4, cytotoxic Tlymphocyte-associated protein 4; CXCL12, chemokine (C-X-C motif) ligand 2; DC, dendritic cell; DCR, disease control rate; dMMR, DNA mismatch repair system; DNA, deoxyribonucleic acid; DNMT, DNA methyltransferase; ECM, extracellular matrix; EpCAM, epithelial cell adhesion molecule; FasL, Fas ligand; FGF, fibroblast growth factor; GAD, glutamate decarboxylase; GCK, glucokinase; GCN2, general control nondepressible-2; GITR, glucocorticoid-induced TNFR family-related protein; GM-CSF, granulocyte macrophage colony stimulating factor; GNS, gold nanostars; HCT, hematopoietic stem cell transplantation; HDAC, histone deacetylases; HVEM, herpes virus-entry mediator; ICD, immunogenic cell death; ICOS, inducible T-cell costimulatory; IDO, indoleamine 2,3-dioxygenase; IFN, interferon; Ig, immunoglobulin; IKK $\beta$, IkB-Kinase $\beta$; IL, interleukin; irAE, immune-related adverse effect; ITIM, immunoreceptor tyrosine-based inhibition motif; KINK-1, kinase inhibitor of NF-kB-1; LAG-3, lymphocyte activation gene 3 protein; LIGHT, lymphocyte activation gene 3 protein; mAbs, monoclonal antibodies; MAPK/ERK, mitogen-activated protein kinase; M-CSF/CSF1, macrophage colony-stimulating factor; MDM2/4, murine double minute 2 homolog; MDR, multidrug resistant; MDSC, myeloid-derived suppressor cell; MHC, major histocompatibility complex; MRD, minimal residual disease; MRI, magnetic resonance imaging; mRNA, messenger RNA; miRNA, micro RNA; MSI-H, microsatellite instability high; mTORC1, mammalian target of rapamycin complex 1; NCP, nano-scale coordination polymer; NFATc1, nuclear factor of activated T-cells; NF-kB, nuclear factor kB; NHL, non-Hodgkin lymphoma; NK, natural killer; NO, nitric oxide; NOTCH1, Notch homolog 1; NSCLC, nonsmall cell lung cancer; ORR, objective response rate; OS, overall survival; OV, oncolytic virus; PAP-1, 5-(4-phenooxybutoxy) psoralen; PD-1, programmed cell death protein 1; PDAC, pancreatic ductal adenocarcinoma; PD-L1, programmed cell death protein 1 ligand; PDT, photodynamic therapy; PDX, patient-derived xenografts; PET, positron emission tomography; PGE2, prostaglandin E2; PI3K $\gamma$, phosphoinositide 3-kinase- $\gamma$; PSGL-1, P-selectin glycoprotein ligand-1; RCC, renal cell carcinoma; ROS, reactive oxygen species; scFv, single-chain variable fragment; Slc15a2, solute carrier family 15, member 2; STAT-3, signal transducer and activator of transcription 3; sTCR, synthetic T-cell receptor; STE, surface Tcell engagers; STING, stimulator of interferon genes; SYMPHONY, synergistic immuno photodermal nanotherapy; T-ALL, T-cell acute lymphoblastic leukemia; TAM, tumor-associated macrophage; TCGA, the cancer genome atlas; TCR, Tcell receptor; TEX, tumor-derived exosomes; TGF, transforming growth factor; TIGIT, T-cell immunoreceptor with Ig and ITIM domains; TIL, tumor infiltrating lymphocyte; TIM-3, T-cell Ig mucin domain-containing 3; TKI, tyrosine kinase inhibitor; TME, tumor microenvironment; TNBC, triple negative breast cancer; TNF, tumor necrosis factor; TNFR, tumor necrosis factor receptor; TNFRSF4 (OX40), tumor necrosis factor receptor superfamily member 4; Treg, regulatory $\mathrm{T}$ cell; T-VEC, talimogene laherparepvec; VEGF, vascular endothelial growth factor; VEGFR, vascular endothelial growth factor receptor; VGSC, voltage-gated sodium channel; VISTA, V-domain Ig suppressor of T-cell activation; ZIKV, Zika virus; ZnP, Zn-pyrophosphate.
}

can be recognized by the host immune-surveillance defensive system, namely natural killer (NK) and T-cells, cancer cells evolve to acquire genetic instabilities and other associated "hallmarks" that can enable immune evasion and persistent growth (1). Host immunity has been shown to detect tumor cell "neoantigens" in vitro. However, neoantigen recognition alone cannot eliminate tumors in vivo given the inherent complex micro-environment, heterogeneous nature and stemness of tumors (Figure 1) $(2,3)$. Indeed, neoantigens are seldom recognized and spontaneously elicit T-cell antitumor responses (4).

An array of normal immune cells, including T-cells, B-cells, and NK cells, together with endothelia, associate with cancer cells and extracellular matrix to form the tumor micro-environment (TME) (Figure 2). This is a dynamic immunosuppressive network and a major obstacle to immunotherapeutic intervention (3). Within TME, adipocytes, regulatory $\mathrm{T}$ (Treg) cells, and fibroblasts, along with a network of cytokines and growth factors, promote cellular proliferation across all stages of tumorigenesis. Thus, both malignant and nonmalignant components of tumors, as well as the mediators of their intercellular communication, are potential targets for immunotherapy (2).

Immune checkpoint receptor pathways represent a major class of "immune synapse," a cell-cell contact that suppresses T-lymphocyte effector functioning (11). This is likely to be an evolutionary countermeasure against autoimmunity, aiming to minimize damage to uninfected cells in virus-infected tissues and to limit systemic inflammation (12). However, tumors can exploit these mechanisms to evade immune detection (Figure 3) $(12,16)$. Hence, such mechanisms provide opportunities for immunotherapy intervention (Figures 4, 5) (19). A plethora of such therapies are currently in preclinical development and clinical application. These include T-cell immune receptor modulating monoclonal antibodies (mAb's), vaccines, adoptive cellular therapy (ACT), engineered oncolytic viruses (OVs), small-molecule targeting drugs, and cytokinebased adjuvant therapies (Table 1). Checkpoint inhibitors, both as monotherapies and in combination, have generated some of the most significant therapeutic efficacies at least in subpopulations of cancer patients $(13,15,22)$. Notably, proof-of-principle has been provided for checkpoint inhibitor mAb's, e.g., anti-CTLA-4 (ipilimumab/Yervoy) and antiPD-1 (nivolumab/Opdivo and pembrolizumab/Keytruda) $(13,22)$. Compared with conventional therapies, these drugs demonstrated significantly higher efficacy and durability as well as reduced toxicity. Importantly, also, a broad spectrum of malignancies could be targeted $(19,22,23)$.

In this review, we explore emerging trends in immunotherapy that are at various stages of development. First, we give an overview of current immunotherapy modalities. Then, we give an account of emerging "next-generation" immune checkpoints and combination immunotherapies. In particular, the latter has surged in popularity since the reports of significantly enhanced treatment efficacy obtained using dual checkpoint blockade with ipilimumab + nivolumab, compared to either drug alone. We also consider the importance of predictive and prognostic biomarkers, including PD-L1, to stratify tumors, boost clinical 


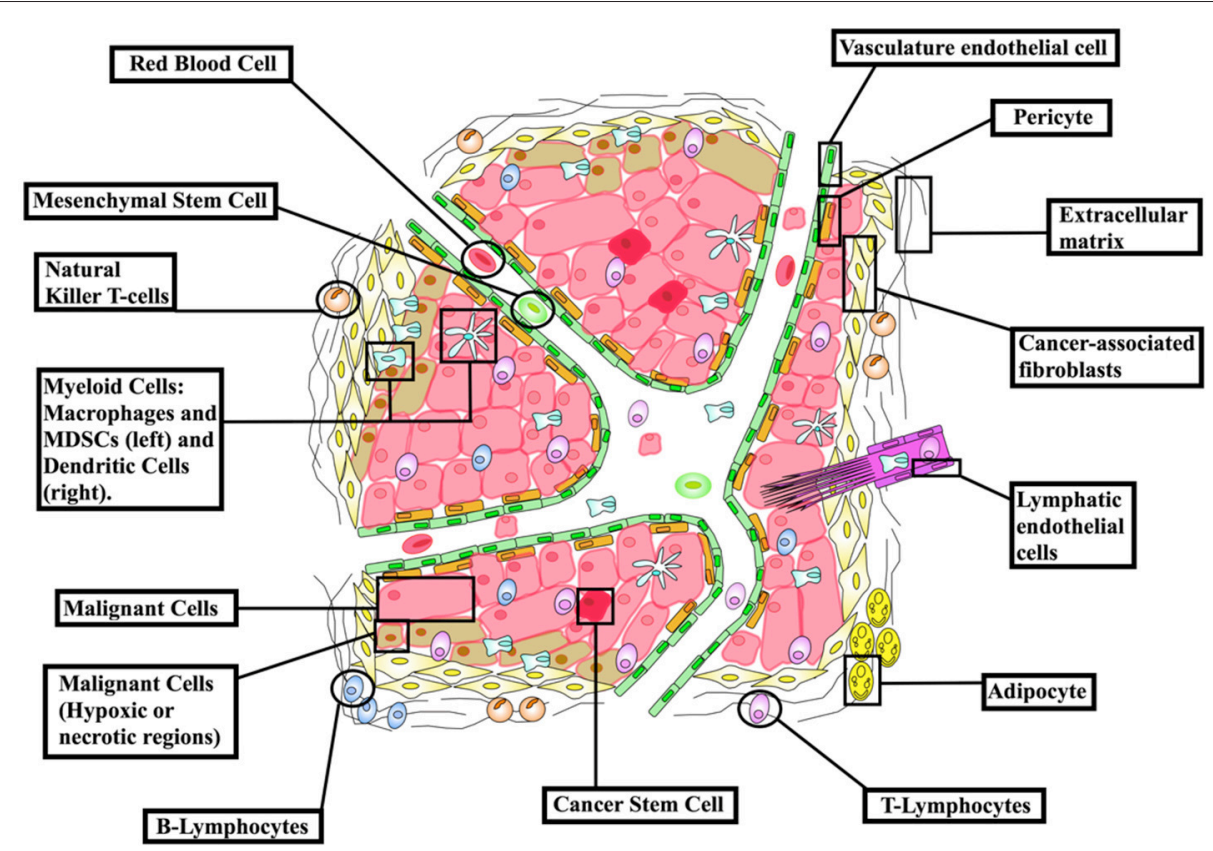

FIGURE 1 | The cellular make-up of the tumor microenvironment (TME). The tumor niche possesses a dynamic structural topography with significant spatial variability in vascular supply, growth factor and cytokine accessibility, ECM-derived structural support and interactions with immune cells. TME hence contributes to tumor heterogeneity as a "rogue organ," formed by normal-malignant cell associations. Created using information from Balkwill et al. (2) and Tang et al. (3).

trial efficacy and increase patient response. Finally, we highlight several categories of promising novel targets that could further enhance the effectiveness of immunotherapy.

\section{CURRENT IMMUNOTHERAPY MODALITIES: AN OVERVIEW}

Immune checkpoint inhibitors currently represent the most promising cancer therapeutics where even monotherapies can produce durable responses in $40-50 \%$ of patients, persisting long after treatment has ceased (Table 1) $(24,25)$. The main strategies are those stimulating effector mechanisms and those neutralizing immunosuppressive mechanisms (16). Vaccinebased oncotherapy using tumor antigen infusion enhances the innate anti-tumor ability of a patient's immune system (26). Additional stimulatory approaches administer genetically engineered OVs to initiate systemic immune responses, use ACT to directly deliver immune cells into patients, or apply costimulatory mAb's specific to members of the tumor necrosis factor receptor (TNFR) superfamily to bolster T-cell function. Immunosuppressive tumor mechanisms include checkpoint inhibitor mAb's targeting inhibitory T-cell checkpoints of PD-1 and CTLA-4, and other targeted antibodies (e.g., against CD25) that deplete inhibitory regulatory Treg cells (16).

Although single-agent immunotherapies, especially checkpoint inhibitors, have demonstrated promising efficacies in some patients with late-stage cancers, however, benefit in most cases was limited (13). In addition, even effective treatments suffered from significant toxicity $(3,25)$. Checkpoint inhibitors can induce pressing "immune-related adverse effects" (irAEs) due to supra-stimulation of immunity. This could impact upon normal adaptability of vital organs such as liver, heart, kidneys, and pancreas and give rise to type 1 diabetes, pancreatitis, arthritis, and lymphocytic myocarditis (27). Also, autoimmune diseases such as hypophisitis, autoimmune hepatitis, pneumonitis, and inflammatory colitis have been reported frequently with use of nivolumab and ipilimumab (27-32). Thus, risk of immune reactions of healthy organs to checkpoint inhibitors remains an understudied area, and immuno-oncologists must tread a "very fine line" between maximizing anti-tumor efficacy and triggering autoimmunity $(27,33)$. More seriously, in a study on a mixed cohort of cancer patients, CTLA-4 or PD-1 blockade was found to induce a 2-fold increase in tumor development and 50\% increase in tumor burden (34). Patients with rare, extra copies of MDM2/4 ("murine double minute 2 homolog") protooncogenes had the greatest risk of such "hyper-progression" (35). In another recent study on a murine model of nonHodgkin's lymphoma, PD-1 signaling prevented cancerous T-cell proliferation, i.e., PD-1 blockade would actually reactivate cancerous T-cells to promote their replication and hence accelerate malignant growth (36). All these highlight the need for profiling individual cancers and patient genomes for best treatment outcome (34-36). Overall, therefore, there are significant limitations in immunomonotherapies given also the intricate heterogeneity and stemness of human tumors (16). Although corticosteroids and supplementary immunosuppressive therapy can help alleviate undesirable 


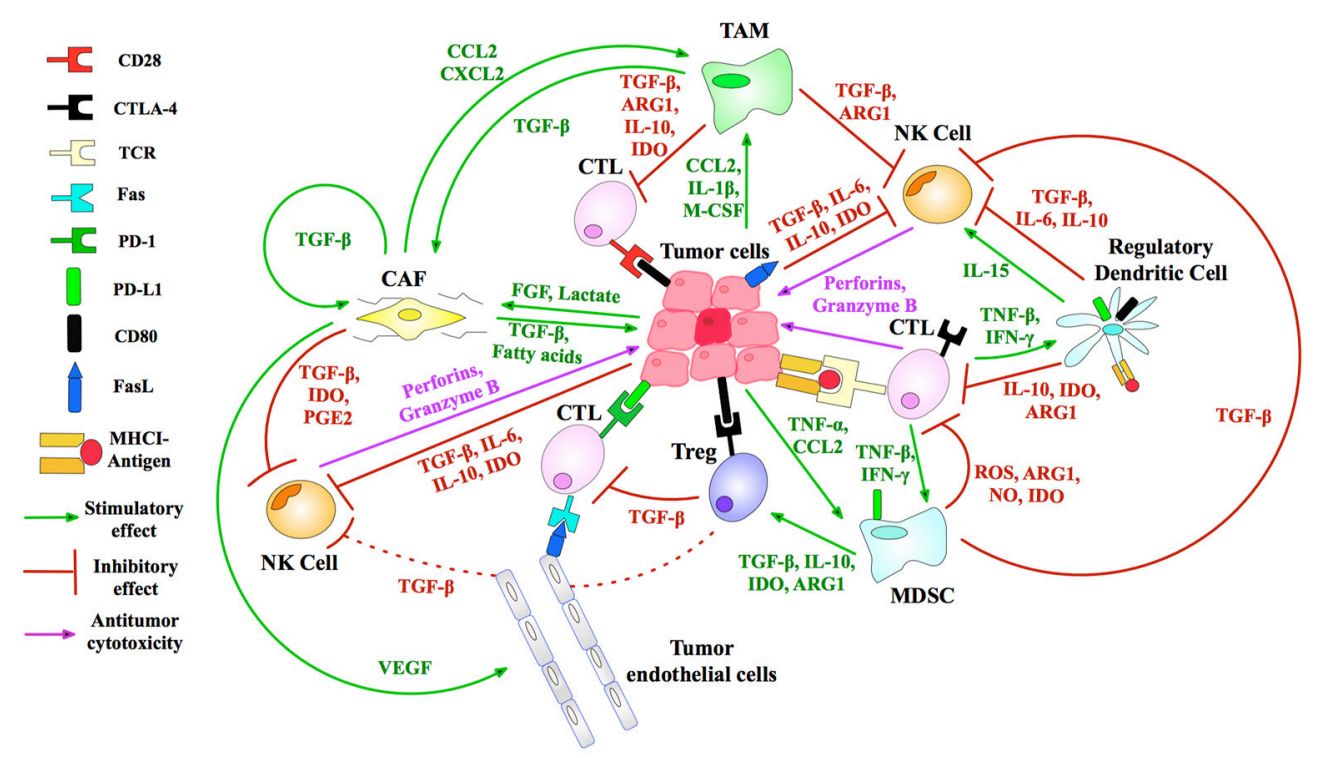

FIGURE 2 | Immunosuppressive mechanisms of the TME. Treg (regulatory T-) cells generate IL-10 and TGF- $\beta$ angiogenic cytokines to suppress CTL (cytotoxic T-lymphocyte) activity. Myeloid-derived suppressor cells (MDSCs) produce reactive oxygen species (ROS), arginase (ARG) and nitric oxide (NO) that inhibit T-cell activation. Tumor-associated macrophages (TAMs) similarly block CTL and natural killer (NK) T-cells, immature dendritic cells cause T-cell anergy via IDO enzyme secretion, while cancer-associated fibroblasts (CAFs) and endothelial cells (tumor, lymphatic, and vascular) produce TGF- $\beta$ and stimulate T-cell apoptosis by FasL-Fas binding $(5,6) . \mathrm{MHC}$ I is downregulated in tumor cells to inhibit T-cell recognition. FasL is expressed by tumors, killing T-cells (7). Tumors secrete VEGF to sustain tumor endothelial cells, and lactate and FGF to promote CAF development (8). Immunosuppressive TAMs are maintained by a suite of tumor secretions: CCL2, CXCL12, and IL-1 $\beta$ (8). NK cell inhibition by tumors is accomplished by release of IL6/10, IDO, and TGF- $\beta$. CAFs suppress NK cells via cytokines and growth factors including PGE2, TGF- $\beta$, and IDO (6). Tumors recruit immunosuppressive to the TME via TNF- $\alpha$ and CCL2 (9). IDO, indoleamine 2,3-dioxygenase; CD80, cluster of differentiation 80; M-CSF, macrophage colony-stimulating factor; CCL2, chemokine ligand 2; PGE2, prostaglandin E2; CXCL2, chemokine (C-X-C motif) ligand 2; TGF, transforming growth factor; IL, interleukin. Figure created by combining information from Jeanbart and Swartz (5), Hargadon et al. (10), Derbal et al. (8), Hasmim et al. (6), and Baginska et al. (9). See Abbreviations list for further definitions.

side effects, it is synergistic "combination immunotherapy" that holds the greatest promise (15, 37-39). Combinations simultaneously targeting different components of tumor development/progression can significantly enhance efficacy, response rates, and durability relative to single-agent firstand second-generation immunotherapies (40, 41) (Figure 6). These "third-generation" novel combinations are increasingly based upon the PD-1/PD-L1 blockade "backbone," given its relatively favorable safety profile and efficacy compared to other checkpoint inhibitors (Table 2) (12, 14, 25, 40, 41). Improved immune targeting and combination therapies owe their enhanced efficacy over monotherapies to the strengthening of multiple components of T-cell anti-tumor responses. This improvement results from (i) functioning of effector T-cells inside TME, including the capacity to evade immunosuppressive checkpoints and soluble factors; (ii) effective extravasation of T-lymphocytes from lymphoid organs into TME; and (iii) production of adequate quantities of effector T-cells inside lymphoid organs (22).

Recent preclinical studies and clinical trials of combination therapies employ immunotherapy coupled with a second immunotherapy modality, as well as chemotherapy or radiotherapy $(13,24)$. Notably, combined checkpoint blockades involving PD-1 or CTLA-4 demonstrated significantly enhanced efficacy against advanced-stage melanoma, relative to targeting each alone (22). Currently, at least 20 single-agent and 3 combination immunotherapy regimens have been approved by the $\operatorname{FDA}(77,78)$. The latter are nivolumab + ipilimumab against melanoma, bevacizumab+interferon-alpha for renal cancer, and elotuzumab + dexamethasone+lenalidomide for multiple myeloma (77). This trend is set to continue with increasing emphasis on rationally designed combinations in personalized settings (14).

\section{EMERGING TARGETS AND COMBINATION THERAPIES}

In the following, we outline emerging targets and possible combinations with checkpoint blockers.

\section{Second Generation Immunotherapy Targets}

Many recent reviews have highlighted emerging alternative checkpoint inhibitors as targets for future monotherapies and/or inclusion in combination therapies $(12,13,15,32,79)$. Whilst CTLA-4 and PD-1 checkpoint inhibitors are the crux of current clinical focus in immunotherapy, other checkpoints with potentially greater potency are emerging and promise to 


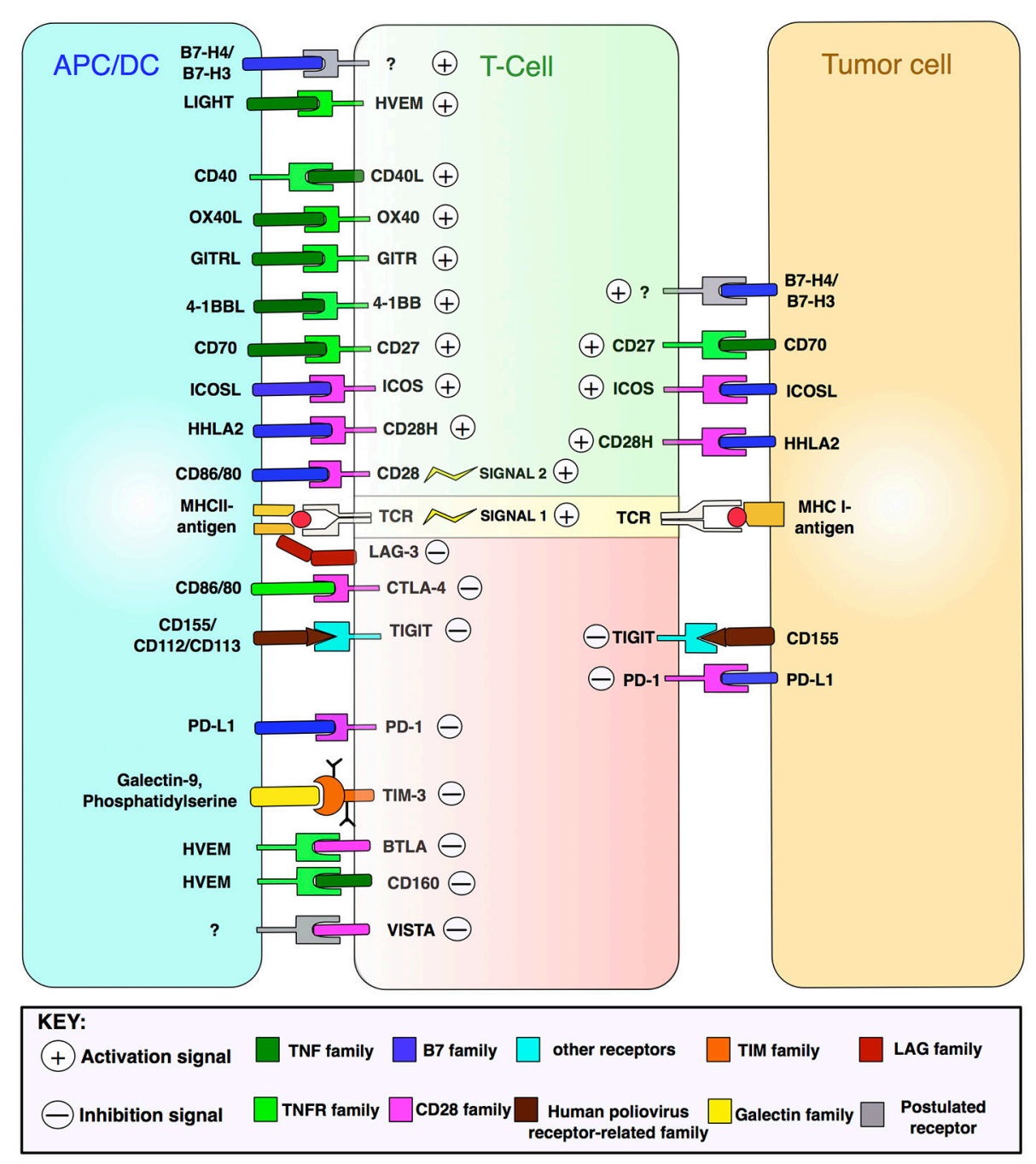

FIGURE 3 | T-cell activation and cell-surface therapeutic targets. T-cell activation by APC/DCs and impact upon the tumor cell is driven by many integrated signals. Depicted are immune receptor-ligand pairings amenable to pharmacological manipulation by immunomodulatory mAbs. HVEM, herpes virus-entry mediator; LIGHT, Iymphocyte activation gene 3 protein; GITR, glucocorticoid-induced TNFR family-related protein; ICOS, inducible T-cell costimulatory; LAG-3, Iymphocyte activation gene 3 protein; TIGIT, T-cell immunoreceptor with Ig and ITIM domains; TIM-3, T-cell Ig mucin domain-containing 3; BTLA, B-lymphocyte and T-lymphocyte attenuator; VISTA, V-domain Ig suppressor of T-cell activation; TNF, tumor necrosis factor. Figure created by combining information from Mahoney et al. (13), Melero et al. (14), and Khalil et al. (15). See Abbreviations list for further definitions.

broaden the therapeutic "toolkit" and improve patient benefit. However, it remains essential to maintain the delicate balance between suppressive and stimulatory checkpoint modulation, using techniques such as multiplex immunoassays (80). VISTA, LAG-3, TIGIT, and TIM-3 immunomodulatory pathways are now well established as novel "next-generation" therapeutic targets (Supplementary Figure 1) (12, 15, 81-86). Most recently, P-selectin glycoprotein ligand-1 (PSGL-1), a glycoprotein with a critical role in cell adhesion and inflammation and regulator of T-cell responses in TME, was also found to be a potential "checkpoint" (87). Notably, ligating PSGL-1 to exhausted CD8+ T-cells inhibited T-cell receptor (TCR) signaling, decreased pro-inflammatory IL-2 and elevated PD-1 levels. Thus, PSGL1 deficiency would reduce PD-1 expression and significantly enhance antitumor T-cell responses to melanoma (87). Anderson et al. postulated (i) that CTLA-4 and PD-1 could serve as "firsttier" co-target receptors responsible primarily for maintaining overall immune self-tolerance and (ii) that "second tier" receptors (TIGIT, LAG-3, and TIM-3), which have overlapping effects on $\mathrm{NK}$ and $\mathrm{CD} 8+\mathrm{T}$-cell effector functions, would exert more specific roles (85). LAG-3, TIM-3, and TIGIT are all highly expressed in dysfunctional T-cells in tumors. Synergizing their corresponding blockades would abrogate Treg cell-mediated immunosuppressive effects and enhance CD8+ and NK cell function within tumor tissues, demonstrating improved safety profiles over CTLA-4 and PD-1 inhibitors. Thus, emerging synergies of first- and second-tier blockades promise to produce stronger responses against a range of malignancies (85). 


\section{A}

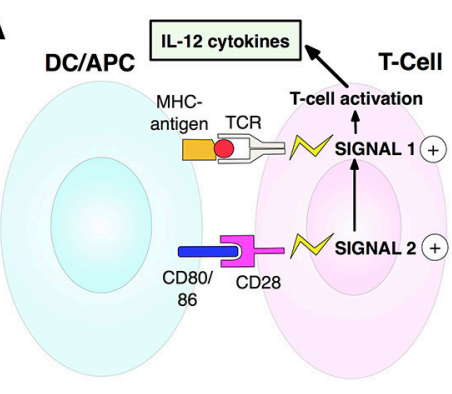

C

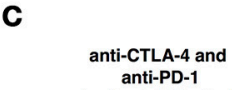
anti-PD-1 DC/APC

mAbs

IFN-y, TNF-a, IFN-y, TNF-a,
granzyme-B cytokines
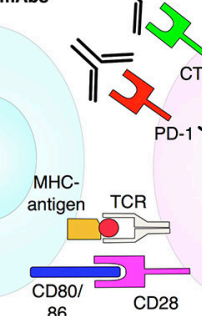

B

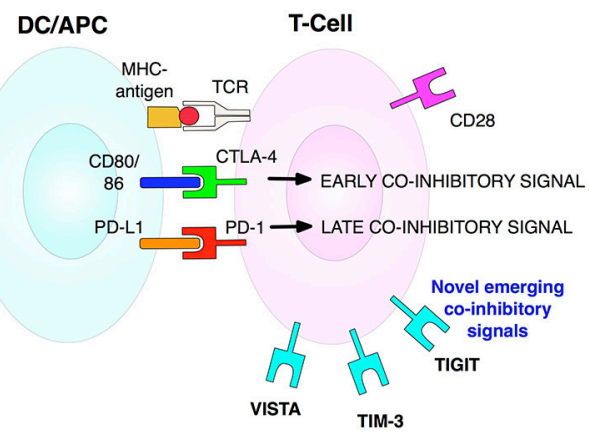

D

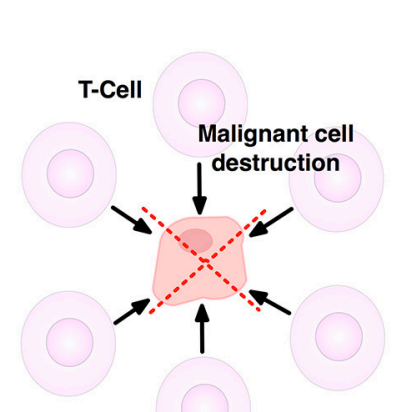

FIGURE 4 | T-cell activation, inhibition and anti-CTLA-4/anti-PD-1 blockade mechanisms. (A) T-cell activation is initiated by TCR-MHCl-antigen interaction (signal 1). Full activation and effector activity demand additional CD28-CD80/86 binding (signal 2). Both signals cause T-cells to secrete IL-2 that drives T-cell proliferation and differentiation. (B) T-cell activation is limited by CTLA-4, upregulated on activated T-cells. CTLA-4 outcompetes CD28 for CD80/86 ligands, thus stopping signal 2 needed for T-cell activation. Contrarily, later coinhibitory PD-1 checkpoint interacts with its ligand to diminish T-cell cytotoxic activity in tumors expressing PD-L1. (C) Dual checkpoint anti-CTLA-4/PD-1 blockade mAbs block inhibitory CTLA-4 and PD-1 checkpoints, enabling release of cytokines involved in sustaining activated T-cells. CD28 can now bind its ligand to enable signal 2. (D) Activated T-cells can now join the antitumor T-cell effector response to destroy tumor cells. Adapted from Mellman et al. (17). See Abbreviations list for further definitions.

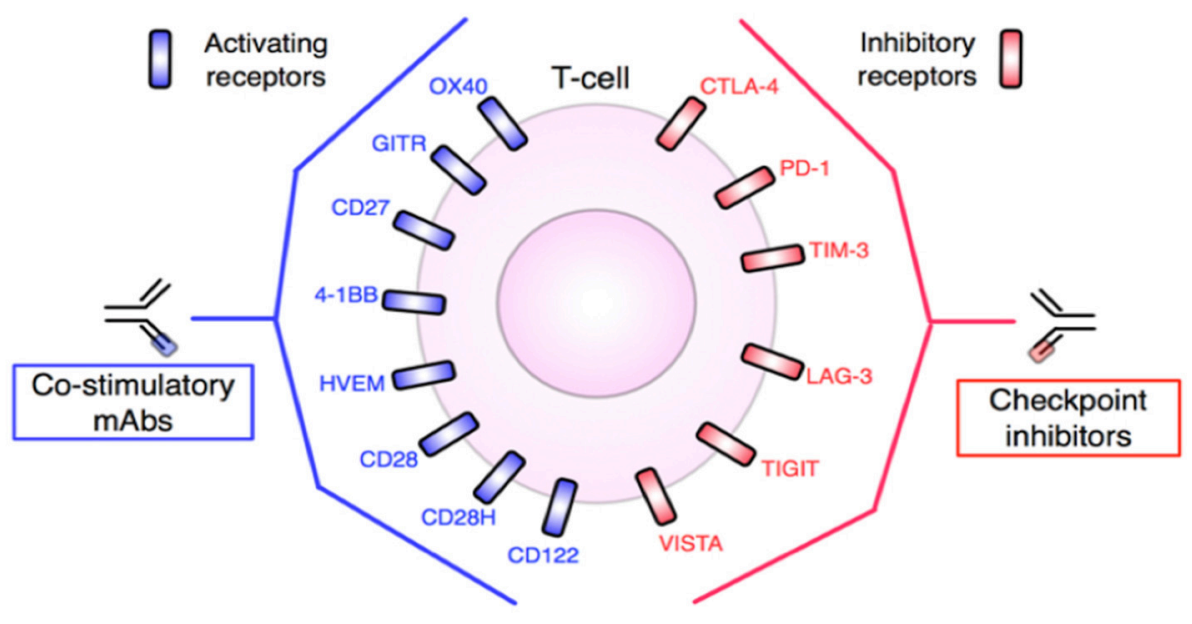

FIGURE 5 | T-cell targets for mAb-based immunotherapy. Inhibitory and stimulatory receptors expressed in the TME may be targeted for therapeutic intervention. Agonistic antibodies, such as anti-OX40 or anti-CD28, target and activate co-stimulatory molecules, while blocking or antagonist antibodies, including anti-PD-1 or anti-CTLA-4, block T-cell inhibitory molecules. In either case, T-cells are stimulated and tumor destruction promoted. Adapted from Mellman et al. (17) and Vasaturo et al. (18). See Abbreviations list for further definitions. 
TABLE 1 | Selected single-agent cancer immunotherapies (15, 20, 21).

\begin{tabular}{|c|c|c|c|c|}
\hline Therapeutic modality & General usage & Current limitations & Example & Development status \\
\hline mAbs & $\begin{array}{l}\text { Very selective agonism } \\
\text { (costimulatory mAbs) or } \\
\text { antagonism/blockade } \\
\text { (checkpoint inhibitors) of immune } \\
\text { receptor-ligand pairings. }\end{array}$ & $\begin{array}{l}\text { Very expensive-over } \$ 120,000 \\
\text { per monotherapy. Time } \\
\text { demanding } \\
\text { manufacture/development. } \\
\text { Challenges in determining ideal } \\
\text { treatment timing and duration. } \\
\text { Need to minimize "on-target, } \\
\text { off-tumor" effects. Often } \\
\text { cancer-specific. Need to identify } \\
\text { more optimal combinations. }\end{array}$ & $\begin{array}{l}\text { Immune checkpoint } \\
\text { blockades/inhibitors: } \\
\text { anti-CTLA-4, anti-PD-1, } \\
\text { anti-PD-L1, anti-LAG3. } \\
\text { Costimulatory mAbs: anti-GITR, } \\
\text { anti-OX40, anti-CD40. }\end{array}$ & $\begin{array}{l}\text { Anti-PD-1 (nivolumab and } \\
\text { pembrolizumab) and } \\
\text { anti-CTLA-4 (ipilimumab) mAbs } \\
\text { are FDA approved for melanoma. } \\
\text { Many others entering clinical } \\
\text { trials for NSCLC, RCC and } \\
\text { kidney cancers. }\end{array}$ \\
\hline Vaccines & $\begin{array}{l}\text { Cancer vaccines introduce } \\
\text { tumor-specific antigens to be } \\
\text { taken up by dendritic or antigen } \\
\text { presenting cells that in turn prime } \\
\text { and boost the T-cell antitumor } \\
\text { immune response. }\end{array}$ & $\begin{array}{l}\text { Many are poorly immunogenic } \\
\text { on their own and require } \\
\text { adjuvants to generate effective } \\
\text { immune responses, but these } \\
\text { adjuvants often increase toxicity. } \\
\text { Low response rates of } \\
\sim 20-30 \% \text {, so need to identify } \\
\text { patient subgroups with a specific } \\
\text { cancer and develop personalized } \\
\text { therapies. }\end{array}$ & $\begin{array}{l}\text { Vaccines targeting gp100, } \\
\text { MUC1, MAGE-A3. Cvac, } \\
\text { BiovaxID, hepcortespenlisimut-L, } \\
\text { and Neuvenge are currently in } \\
\text { development. }\end{array}$ & $\begin{array}{l}\text { FDA-approved vaccines include: } \\
\text { Oncophage for kidney cancer, } \\
\text { and sipuleucel-T for metastatic } \\
\text { prostate cancer. Many are } \\
\text { entering clinical development, } \\
\text { and several e.g., BiovaxID are in } \\
\text { phase III trials. }\end{array}$ \\
\hline Small molecules & $\begin{array}{l}\text { Uniquely specialized for specific } \\
\text { intracellular targets, but also } \\
\text { suitable for extracellular or cell } \\
\text { surface targets. }\end{array}$ & $\begin{array}{l}\text { Off-target activity/lack of } \\
\text { specificity, dose-limiting toxicity, } \\
\text { not effective at blocking immune } \\
\text { protein-protein interactions. } \\
\text { Often demands daily } \\
\text { administration. }\end{array}$ & $\begin{array}{l}\text { Inhibitors of VEGF } \\
\text { (bevacizumab), HDAC } \\
\text { (entinostat), DNMT } \\
\text { (5-azacytidine). TKIs (imatinib), } \\
\text { BRAF mutant inhibitor } \\
\text { (vemurafenib). IDO1 and COX2 } \\
\text { inhibitors. }\end{array}$ & $\begin{array}{l}\text { IDO, HDAC, DNMT, VEGF, and } \\
\text { TK inhibitors are in clinical trials. } \\
\text { Several efficacy trial results are } \\
\text { awaited. }\end{array}$ \\
\hline Adoptive T-cell therapy & $\begin{array}{l}\text { Tumor-targeted cytotoxicity } \\
\text { against both intracellular and } \\
\text { extracellular tumor-specific } \\
\text { antigens. }\end{array}$ & $\begin{array}{l}\text { Tumor heterogeneity - mutated } \\
\text { antigen expression and } \\
\text { composition impacts response } \\
\text { rates. "On-target, off-tumor" } \\
\text { toxicity issue. Ultimately, need to } \\
\text { translate deep remissions into } \\
\text { cures by fine-tuning regimens } \\
\text { and targeting many antigens } \\
\text { simultaneously. Commercial } \\
\text { mass production difficult. }\end{array}$ & $\begin{array}{l}\text { Tumor-infiltrating lymphocytes } \\
\text { (against mutated EBR2) for bile } \\
\text { duct cancer, genetically } \\
\text { engineered CARs (against CD19) } \\
\text { for lymphoma and genetically } \\
\text { engineered T-cells with sTCRs } \\
\text { (against MART-1) for melanoma. }\end{array}$ & $\begin{array}{l}\text { Clinical trials have been } \\
\text { accelerating since } 2010 \text {, with } \\
\text { over } 20 \text { clinical trials in progress } \\
\text { against melanoma, lymphoma } \\
\text { and leukemias. }\end{array}$ \\
\hline Cytokines & $\begin{array}{l}\text { Agonism or antagonism of } \\
\text { immune protein-protein } \\
\text { pathways. }\end{array}$ & $\begin{array}{l}\text { Generally high toxicities. } \\
\text { Poor pharmacokinetics. }\end{array}$ & $\begin{array}{l}\text { GM-CSF, IL-12, IL-15, IL-21, } \\
\text { IFN- } \gamma \text {, and TNF- } \alpha .\end{array}$ & $\begin{array}{l}\text { IL-2 is now approved for } \\
\text { metastatic melanoma and RCC. } \\
\text { IFN-alpha approved for stage III } \\
\text { melanoma. }\end{array}$ \\
\hline Oncolytic viruses & $\begin{array}{l}\text { Exploits viral ability to replicate } \\
\text { and kill tumor cells while } \\
\text { simultaneously stimulating } \\
\text { patient-specific antitumor } \\
\text { immune responses. }\end{array}$ & $\begin{array}{l}\text { Need to better characterize } \\
\text { mechanisms of action, develop } \\
\text { combinatorial immunotherapies, } \\
\text { and scale-up for mass } \\
\text { commercialization. }\end{array}$ & $\begin{array}{l}\text { Talimogene laherparepvec or } \\
\text { T-VEC used for melanoma. }\end{array}$ & $\begin{array}{l}\text { T-VEC became FDA-approved in } \\
2015 \text { as Imlygic for patients with } \\
\text { inoperable cancers. Other phase } \\
\text { I trials are ongoing for pancreatic, } \\
\text { breast and colorectal cancers. }\end{array}$ \\
\hline
\end{tabular}

\section{Dual T-Cell Checkpoint Blockade}

Rationale for synergizing anti-PD- 1 and anti-CTLA-4 inhibitory mAb's is strong since both are expressed on T-cells but employ distinct, complementary mechanisms of action for suppressing T-cell function (Figures 4A-D) (12). A phase I trial of nivolumab (anti-PD-1) and ipilimumab (anti-CTLA-4) combination on patients with advanced melanoma produced an unprecedented $53 \%$ objective response rate (ORR), leading to two-year overall survival (OS) in 79\% of cases (NCT01927419) (43). Later studies corroborated the findings in showing the superiority of the combination (ORR $=61 \%)$ compared with ipilimumab alone (11\%); $22 \%$ of patients showed total remission (22). In phase III trials, anti-PD-1 alone, and anti-PD-1+anti-CTLA-4 combination were less toxic and had higher efficacy than anti-CTLA-4 alone. However, more cases of irAE were reported in the combination group relative to ipilimumab or nivolumab monotherapies (55 vs. $27 \%$ and $16 \%$, respectively) (44). These included severe acute tubulointerstitial nephritis and a systemic rash but could be reversed with immunosuppressants and no fatalities occurred (NCT01844505) (44).

Prostate cancer is thought to be immunologically "cold," lacking in tumor infiltrating lymphocytes (TILs), thereby limiting the effectiveness of PD-1 blockade which favors high-TIL tumors 


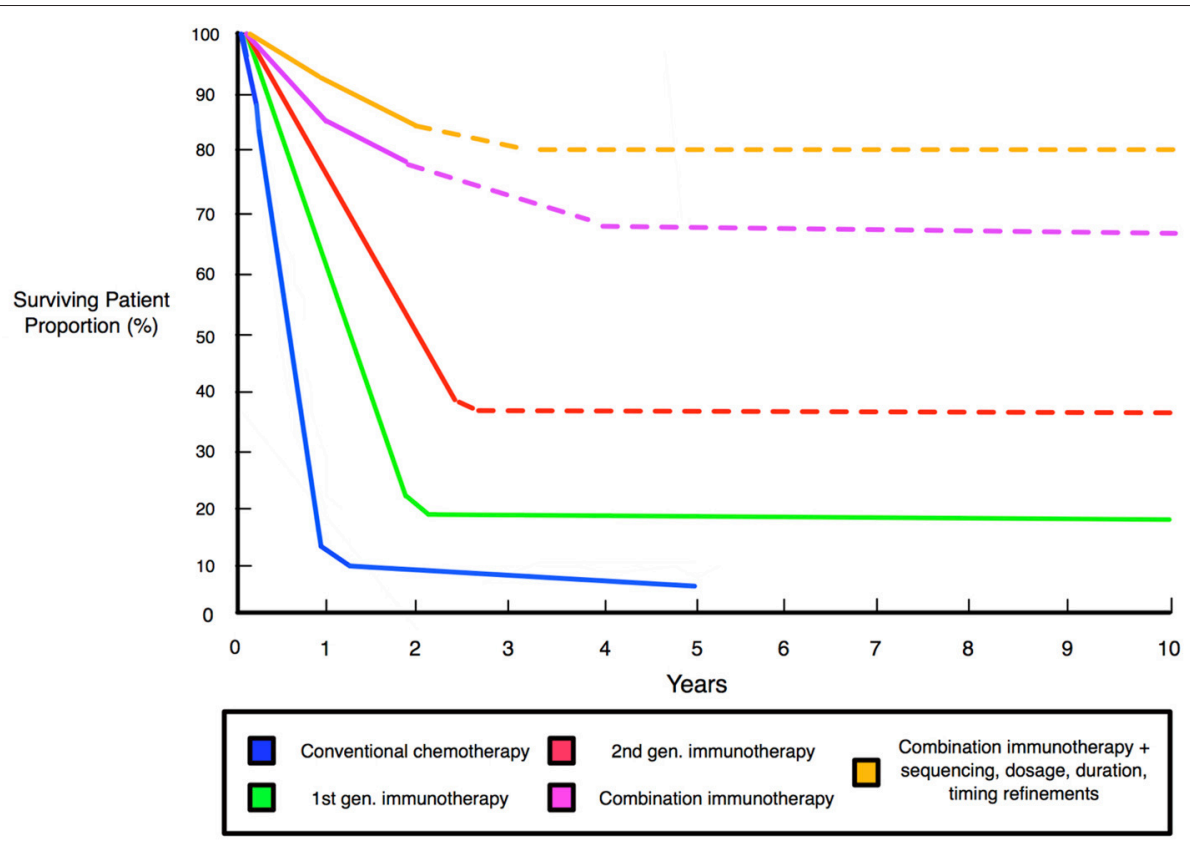

FIGURE 6 | Schematic comparison of patient survival associated with different therapies and improved survival with combination immunotherapy. Graph shows significantly improved survival for immunotherapies relative to conventional chemotherapy. First generation immunotherapies entail anti-CTLA-4 ipilimumab and the therapeutic vaccine Sipuleucel-T that defined the initiating wave of modern immunotherapies. Second generation immunotherapies are exemplified by anti-PD-1 nivolumab and pembrolizumab, and anti-PD-L1 agents of durvalumab and atezolizumab, that deliver effective responses in $40 \%$ of patients across many clinical trials (42). Combinations, such as dual-checkpoint CTLA-4/PD-1 blockade, produce strong effects in 60-70\% of patients and alongside multifunctional single-agent modalities, represent the "third generation" of immunotherapies $(40,41)$. Dashed lines indicate projected survival rates based upon preclinical and clinical trials.

(88). Recently, however, Sharma et al. treated prostate cancer patients with anti-CTLA-4 (ipilimumab) and observed both elevated levels of T-cells in tumors and increased expression of PD-1 and VISTA inhibitory checkpoints. Consequently, combinations of checkpoint inhibitors targeting CTLA-4, PD-1, and VISTA would appear promising (88).

\section{Costimulatory mAb's}

This approach aims to generate synergies between checkpoint inhibitors and costimulatory receptor mAb's (Figure 5). The first signal necessary for T-cell activation is triggered when APCs present antigens to TCRs via MHCs. The second/final signaling occurs when co-stimulatory receptors on T-cells (e.g., CD28) interact with compatible APC surface proteins (Figure 3). Progress in this approach was initially slow, owing to the clinical failure of the CD28 super-agonist mAb TGN1412 that induced "cytokine storms" and life-threatening organ failure in $17 \%$ of patients (89). More recent, promising trials incorporate agonist mAb's targeting costimulatory receptors including 4$1 \mathrm{BB}$, GITR and OX40, that promote proliferation and survival of T-cells (12). Other "receptors" include CD27 (involved in long-term immunological memory of T-, B- and NK-cells) and CD40 (mediating antigen-presenting cell activation) (14). A phase I trial on metastatic melanoma patients $(n=24)$ with tremelimumab (anti-PD-1) and CP-893,870 (a CD40-agonist $\mathrm{mAb}$ ) led to $27 \%$ ORR, 26-month OS and complete response in $8 \%$ of cases. Although $79 \%$ of patients developed cytokine release syndrome, this could be managed by standard care (NCT01103635) (51).

Co-stimulatory agonist mAb's targeting T-cell antigen 4$1 \mathrm{BB}$ are among the most advanced to be developed (15). This antigen is appealing given its expression on both T-cells and APCs, coupled with its ability to boost T-cell effector functions, expansion, and survival (90). In a murine colon adenocarcinoma model, significant synergy was reported for 4-1BB agonists plus PD-1 blockade combination resulting in total rejection of tumors (91). This effect involved increased levels of intra-tumor IFN $\gamma$-producing CD8 + and CD4+ Tcells, compared to monotherapies. Furthermore, the extent of irAEs was much improved and there was no overt toxicity (91). A further study on mice showed, however, that while 4-1BB mAb agonists alone halted progression of c-Mycdriven B-cell lymphoma in $70 \%$ of cases, combination of 4$1 \mathrm{BB}$ agonist with PD-1 blockade unexpectedly reduced this antitumor effect (92). Furthermore, concurrent PD-1 blockade and OX40 agonist in breast cancer murine models dampened the efficacy of OX40 agonist, with significantly reduced $\mathrm{CD}^{+}{ }^{+}$and $\mathrm{CD}^{+}$lymphocyte infiltration, whilst applying OX40 agonist followed by PD-1 blockade enhanced efficacy, regressing breast tumors in $30 \%$ of cases (93). Consequently, simultaneous modulation of costimulatory and coinhibitory T-cell receptors warrants further investigation with careful consideration to the timing of the combination treatment (92). 
TABLE 2 | Selected key combinations of immunotherapies in preclinical and clinical development with checkpoint blockade as "backbone."

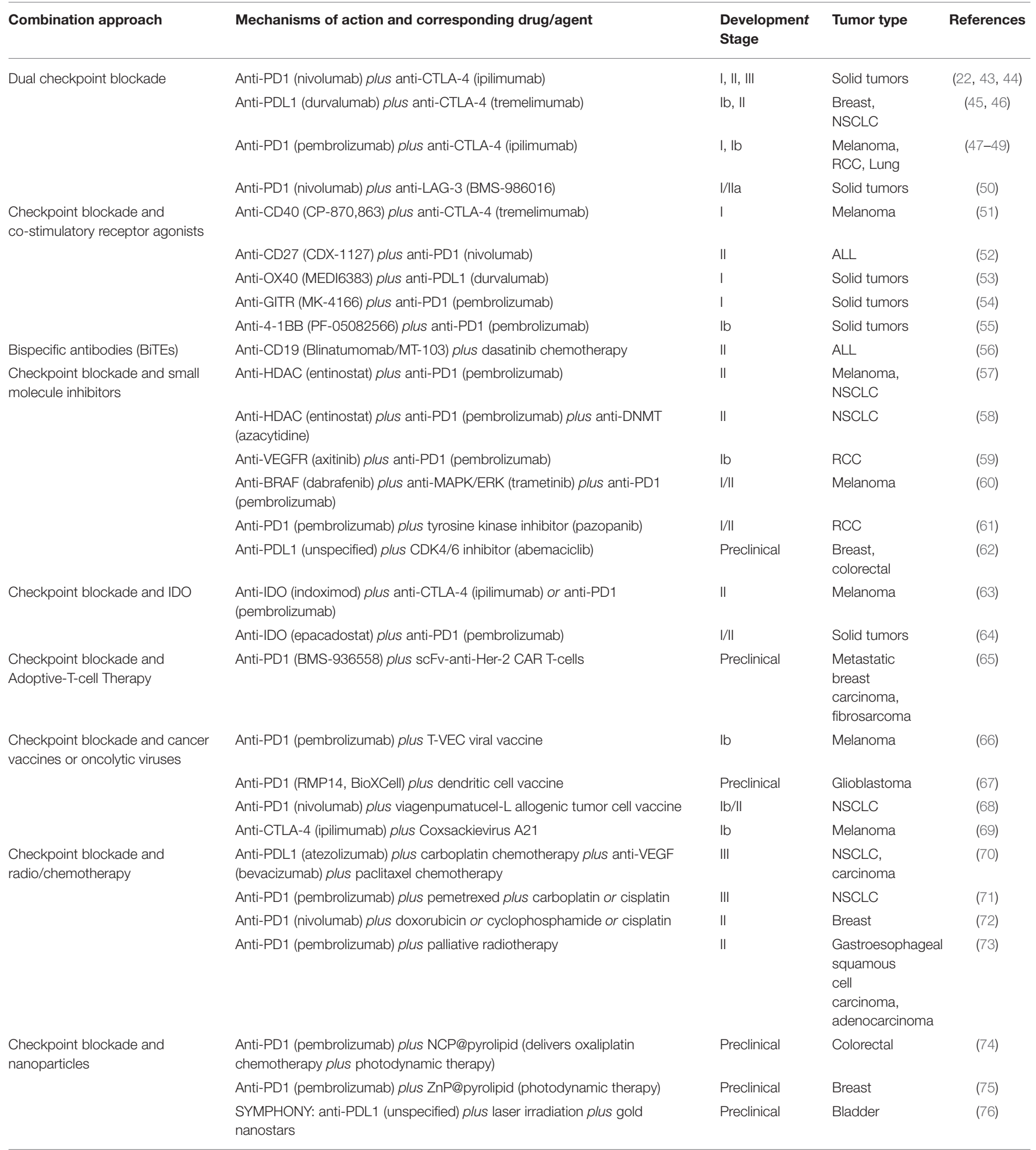

\section{Checkpoint Blockers With Conventional Therapies}

Radiotherapy results in stimulation of DNA-damage repair mechanisms and release of proinflammatory cytokines and tumor antigens (12). Localized radiotherapy (even sub-therapeutic dosages) can also cause significant immunostimulatory regression of distant, non-irradiated tumors, known as an "abscopal effect." The latter was exploited 
in a combination with checkpoint blockers (ipilimumab or pembrolizumab) against metastatic melanoma (94). Such coupling of checkpoint inhibitors with radiation significantly enhanced tumor CTL infiltration and elevated ORR in prostate cancer, NSCLC and glioblastoma (95). Furthermore, only lowmoderate toxicity ( $\sim 10 \%$ irAEs) was reported for combination of PD-1 or CTLA-4 blockade with radiotherapy against metastatic lung cancer (96). Interestingly, a triple combination of anti-CTLA-4 + anti-PD-1 + radiotherapy induced complete responses in mouse pancreatic cancer and melanoma models, not seen with dual-checkpoint blockade alone (13). In certain cases, however, radiotherapy + anti-CTLA- 4 of patients with high tumor PD-L1 levels (type I TME) did not respond, contrary to anti-PD-1 treatment alone. Hence, future trials combining anti-PD-1 and radiotherapy could enhance ORR especially in patients possessing TMEs rich in PD-L1 expression and CD8+ lymphocyte infiltration $(13,24,97)$.

Chemotherapy can also promote anti-tumor immune response by stimulating proinflammatory cytokines, reducing cytotoxic T-cell loss, and specific immunomodulatory effects (98). Examples of the latter include myeloid-derived suppresor cells (MDSCs) and Treg cell depletion by taxanes and cyclophosphamide, respectively (12). A phase $\mathrm{Ib}$ trial on advanced or metastatic NSCLC patients found that atezolizumab followed by carboplatin/nab-paclitaxel induced a response rate of $75 \%$ ( $c f$. $\sim 30 \%$ obtained with single-agent platinum doublet treatment) (NCT00527735) (99). More recently, a chemo-immunotherapy approach to murine colorectal cancer, combining oxaliplatin with PD-1 or CTLA-4 blockade proved synergistic by generating high levels of TILs and pro-inflammatory cytokines and downregulating inhibitory checkpoints (100).

A primary clinical objective is to convert "cold" nonimmunogenic tumors into "hot" immunogenic tumors more receptive to immunotherapy by priming T-cells already present (101). In this regard, chemotherapy-based immunomodulation before checkpoint blockade shows promise. In a phase II trial, 50 metastatic triple negative breast cancer (TNBC) patients were given low-dose chemotherapy (over 2 weeks) followed by nivolumab. This produced ORRs of $24 \%$ and OS of $80 \%$ after 1 year, with an acceptable toxicity level, superior to existing anti-PD-1 monotherapies (NCT02499367) (72, 101). Thus, such "one-two-punch" strategies of low-dosage immunogenic chemotherapies plus checkpoint blockers (or cell cycle inhibitors or epigenetic modulators) can boost tumor immunogenicity. In turn, this promises to avert tumor relapse by destroying dormant cancer cells and/or enforcing their prolonged dormancy, and could be incorporated into future combination therapies (102). Indeed, a recent phase III trial, treatment of non-squamous metastatic NSCLC patients $(\mathrm{n}=616)$ with pembrolizumab plus chemotherapy (cisplatin/carboplatin and pemetrexed) showed greatly improved efficacy relative to chemotherapy alone $(\mathrm{ORR}=48$ vs. $19 \%)$ with no change in the irAE level (NCT02578680) (71). Interestingly, OS was improved regardless of tumor PD-L1 expression levels, even in PD-L1 negative patients. This result would argue strongly for pembrolizumab + chemotherapy combination replacing chemotherapy alone as the standard of care for first-line treatment of metastatic NSCLC (71).

\section{Bifunctional Agents}

These include bispecific antibodies (bsAb's) and doubleheaded fusion proteins. bsAb's have dual specificity, binding simultaneously to two antigens, and high affinity (103). Bispecific T-cell engagers (BiTE's) represent an innovative format comprising two single-chain variable fragments (scFv's) joined in tandem via a flexible linker, where one antibody is specific for CD3 (a surface co-receptor on T-cells) and the other for a selected antigen on malignant target cells (104). Blinatumomab, the first FDA-approved bsAb/BiTE, binds T-cell CD3 and CD19-expressing B-cell acute lymphoblastic leukemia (B-ALL), thus eliminating tumors by redirecting T-cells onto them. The subsequent influx of granzyme proteases (derived from T-cells) enables a cytosolic synapse between T-cells and target cells, inducing apoptosis of B-cells (105); T-cells also proliferate and secrete pro-inflammatory cytokines including IL-2, TNF $\alpha$, and IFN $\gamma$ (105). In a phase II trial against refractory B-ALL, 43\% of patients showed complete responses and a median OS of 6.1 months (NCT01466179) $(106,107)$. Blinatumomab and other BiTE antibodies aim to overcome tumor immune evasion mechanisms by directly engaging endogenous T-cells $(104,105)$. This could prevent the need (i) to expand and reintroduce T-cells, including specific clones, ex vivo, and (ii) to use costimulatory molecules $(104,105)$. Significant advantages over standard mAb's include enhanced cytotoxic potential, ability to bind weaklyexpressed tumor antigens, superior protein stability and high potency in redirecting T-cells to target tumors even at low dosages $(10-100 \mathrm{pg} / \mathrm{ml})(104,105,108)$.

Cancer stem cells (CSCs) play a significant role in tumor initiation and progression, and their eradication is critical for preventing chemoresistance and eventual disease recurrence (109). The single-chain BiTE Solitomab (MT110) simultaneously targets the epithelial cell adhesion molecule (EpCAM) CD326, a transmembrane glycoprotein and promising CSC biomarker, and CD3 on T-cells (109). In a mouse model of human pancreatic CSCs, MT110 stabilized tumor growth and small remaining tumors contained no CSCs (109). BiTEs are being developed for a range of hematological and solid tumors, including ALL, non-Hodgkin lymphoma (NHL), glioblastoma, melanoma, and cancers of breast and prostate (109). However, some side effects of cytokine release syndrome (CRS) have been reported, and benefits appeared short-lasting possibly due to the small size of BiTEs $(\sim 55 \mathrm{kDa}) /$ short half-lives, requiring repeated administering every $48 \mathrm{~h}$ (110). BiTE performance is also being evaluated in combination with anti-PD-1 + anti-CTLA-4 immune blockades to enable even greater T-cell activation (103).

Bispecific fusion proteins (created by joining parts of two different genes) are being used to simultaneously to block PD1/PD-L1 and growth factor/cytokine signaling. A first such protein (M7824) has recently been investigated in phase I trials against several types of advanced solid tumors and has produced promising complete or partial response rates of up to $21 \%$ (NCT02517398, ongoing) (111). M7824 simultaneously blocks PD-L1 and TGF- $\beta$ immune-inhibitory pathways to both restore 
and enhance host immune responses. The rationale is based upon averting the immunosuppression of effector T-cell function by PD-L1 and sequestering TGF- $\beta$ (secreted by malignant cells, MDSCs, and Treg cells), hence preventing TGF- $\beta$-mediated tumor development and metastasis (111).

\section{Epigenetic Modulators}

Here, a checkpoint inhibitor is combined with an epigenetic modulator, such as an inhibitor of histone deacetylases (HDAC) or DNA methyltransferase (DNMT). This is viable since HDAC is commonly overexpressed in tumors and its inhibition downregulates the expansion of MDSCs that normally accompanies and promotes the cancer process (112). Additionally, most epigenetic drugs demonstrate only minor toxicity at clinical dosages (113). A major study focused on complementing the high-efficacy/short-term effects of targeted inhibitors with the low response rate/durable efficacies of single-agent immunotherapies (12). Mouse carcinoma models were used to examine the efficacy of ipilimumab (anti-CTLA4) and nivolumab (anti-PD-1) synergized with 5-azacytidine (DNMT inhibitor) and entinostat (HDAC inhibitor). This eliminated $>90 \%$ of colorectal carcinomas and $100 \%$ of metastatic mammary tumors. Rather disappointingly, however, phase II trials against NSCLCs reported durable, complete responses in only $\sim 5 \%$ of patients $(112,114)$. 5-azacytidine also boosted antitumor immunity by dampening Treg cell function and upregulation of tumor cell antigen presentation machinery, critical to effective immune responses (115). Notably, demethylation of T-cell PD-1 promoters by 5 -azacytidine in AML patients correlate with upregulated T-cell PD-1 expression (116). Consequently, DNMT inhibitor + PD-1 blocker combinations are being explored in ongoing clinical trials against NSCLC and other malignancies (115).

\section{Checkpoint Blockers With Targeted Therapies}

In this combination, checkpoint inhibitors are coupled with a modulator of growth factor signaling, mainly an inhibitor of protein kinase or phosphatase. In particular, receptor and nonreceptor tyrosine kinases play a significant role in tumorigenesis as well as in immunogenicity and cytotoxicity $(12,117)$. Consequently, their inhibitors (TKIs) would offer natural synergy with checkpoint blockers. The angiogenesis-inducing growth factor, VEGF, restricts T-cell infiltration across the tumor endothelium and amplifies MDSCs and Treg cells within tumors. Against metastatic melanoma, combination of bevacizumab (a VEGF inhibitor) with ipilimumab induced a disease-control rate (DCR) of $67 \%$ and promoted T-lymphocyte infiltration of tumors with favorable tolerance. Combinations are now being sought that might synergize anti-PD-1/PD-L1 mAb's with VEGF blockade for even greater efficacy $(22,79)$.

In a mouse model of gastrointestinal stromal tumor (GIST), imatinib (a broad-spectrum TKI) was combined with an antiCTLA-4 mAb to block T-cell immunosuppression mediated by indoleamine 2,3-dioxygenase (IDO). Synergistic activity was reported that reduced Treg cell population and enhanced tumor infiltration by CD8+ T-cells. Thus, CTLA- 4 and IDO blockade combination significantly decreased tumor volume by $50 \%$ after 80 days, while during CTLA-4 blockade and imatinib administration alone tumors expanded by $40-60 \%$ over a similar period (117).

Ser/thr kinase and proto-oncogene BRAF promotes cell proliferation. In $\mathrm{BRAF}^{\mathrm{V} 600 \mathrm{E}}$ mutant melanomas, the specific inhibitor vemurafenib elevated MHCI induction and blocked immunosuppressive cytokine secretion (118). Unfortunately, however, a phase I vemurafenib+ipilimumab trial was canceled after significant level of irAE was reported (119). Later studies employed an extended combination (a BRAF inhibitor + a PD-L1 blocker + a MAPK/ERK inhibitor) to yield vastly improved efficacy $(79,120)$. Furthermore, numerous cancers express IDO that represses anti-tumor responses by depleting tryptophan-critical for T-cell effector activity and survivaland produce immunosuppressive metabolites. Accordingly, therapeutic interventions with PD-1 and IDO blockades have generated significant clinical potency and sustained objective responses. In phase II trials against advanced melanoma, a synergistic combination of the IDO inhibitor indoximod with pembrolizumab produced complete or partial responses in 52\% of patients, with negligible toxicity (NCT01866319) (63). Relative to PD-1, a superior safety profile was reported with CTLA4 blockade. Another IDO inhibitor, epacadostat, was recently combined with pembrolizumab against metastatic melanoma and demonstrated $74 \%$ DCR and 53\% ORR, with only 5\% experiencing irAEs (NCT02178722) (64).

Macrophage phosphoinositide 3-kinase- $\gamma$ (PI3K $\gamma$ ) serves as a critical regulator of immune suppression, supporting immunosuppressive myeloid cells within TME (121). In preclinical trials, inhibition of PI3K $\gamma$ with IPI-549 resulted in reprogramming of immunosuppressive macrophages (M2) into a pro-inflammatory (M1) state. This enhanced both activation and recruitment of cytotoxic T-cells to melanomas. Mice with melanoma rich in macrophages had dramatically enhanced response to immune checkpoint inhibitors and survival when co-treated with IPI-549: Monotherapy with anti-CTLA-4 or antiPD-1 produced total remission in $20 \%$ of cases whilst addition of IPI-549 increased this to $80 \%$. We should note, again, however, that IPI-549 would show benefit only against tumors with high myeloid cell content, so appropriate preselection of patients would seem essential for the best possible outcome of the cotreatment (122).

Colony-stimulating factor 1 (CSF1/M-CSF) also contributes to resistance of melanoma to PD-1 blockade (123). Activated CD8+ T-cells, upon releasing IFN- $\gamma$ and TNF- $\alpha$ into the TME, experience a "vicious cycle" whereby these immunosuppressive cytokines trigger melanoma to adaptively secrete CSF1 (123). In turn, CSF1 promotes the differentiation and accumulation of pro-tumoral/inflammatory TAMs and MDSCs. In murine melanoma models driven by BRAF ${ }^{\mathrm{V} 600 \mathrm{E}}$, anti-CSF1 inhibitors alone displayed modest efficacy, yet dual blockade of antiCSF1 and anti-PD-1 regressed 100\% of tumors by 17 days, with $90 \%$ survival after 90 days (123). CSF1 inhibitors also showed additive effects with ACT therapies in preclinical melanoma studies, where anti-CSF1 dampened myeloid cellmediated immunosuppression in the TME, permitting greater 
CD4+ and CD8+ T-cell infiltration into tumors and with improved functionality (124). Such effects could be beneficial to melanoma patients refractory to existing checkpoint blockade and ACT monotherapies.

IKK $\beta$ (IkB-Kinase $\beta$ ) represents a major component of the NF-kB signaling pathway, responsible for mediating $\mathrm{T}$ cell development and activation (125). Mature Treg cells avert autoimmunity yet limit antitumor immune responses via CTL inhibition, and are heavily reliant upon NF-kB signaling for their development. Consequently, in melanoma murine models, IKK $\beta$ inhibition with KINK-1 (Kinase Inhibitor of NF-kB1) reduced circulating Treg cells by $\sim 50 \%$ with no change in CTL levels (125). The latter is due to CTLs being less reliant upon IKK $\beta$ for proliferation and survival than Treg cells. Thus, combining Treg-nullifying IKK $\beta$ inhibitors with other immunoactive pharmacological agents could bolster therapeutic efficacy (125).

Cyclin-dependent kinases 4 and 6 (CDK4/6) are core cellcycle components, essential to initiation and development of breast cancer and T-ALL. CDK4/6 inhibitors (CDK4/6i) showed effectiveness against glioblastoma, breast cancer and melanoma by arresting tumor cell cycle at G1, via inhibition of retinoblastoma tumor suppressor phosphorylation (126). CDK4/6i can also induce antitumor immunity by overcoming two tumor immunoevasion mechanisms via (i) presenting tumor surface antigens with enhanced efficiency and (ii) inhibiting immunosuppressive Treg cell proliferation (62). Indeed, in murine breast cancer models, abemaciclib (CDK4/6i) + antiPDL1 reduced tumor volume by $70 \%$ after $\sim 2$ weeks (stable up to 35 days) while abemaciclib or anti-PDL1 monotherapy was effective only temporarily. In colorectal CT-26 mice models also, this combination produced prolonged $100 \%$ regression, accompanied by resistance to further disease inducation (62).

\section{Checkpoint Blockers With Cancer Vaccines}

Sipuleucel-T (the only FDA-approved cancer vaccine) monotherapy has limited efficacy, probably due to T-cell inactivation resulting from TME-induced immunosuppression (12). In contrast, combination with checkpoint inhibitors appears promising, and Sipuleucel-T+ ipilimumab is currently in phase II trials against chemotherapy-naive prostate cancer (127). No benefit was reported from addition of nivomulab to a multipeptide vaccine against melanoma (12). On the other hand, poly(lactide-co-glycolide) vaccines combined with anti-CTLA-4 checkpoint inhibitors demonstrated promising results, regressing B16 melanoma tumors in mice and increasing survival rates by $75 \%$ (128). Another preclinical study showed that whole-tumor vaccines producing granulocyte macrophage colony-stimulating factor (GM-CSF) greatly enhanced anti-CTLA-4 blockade efficacy against pancreatic and prostate cancers. Compared with monotherapy, ipilimumab+sagramostim (recombinant GM-CSF) improved OS by $38 \%$ and reduced toxicity (37). Furthermore, whilst checkpoint inhibitor monotherapies proved ineffective against pancreatic ductal adenocarcinoma (PDAC) in mice, anti-PD1 blockade coupled with GVAX vaccine (secreting
GM-CSF) improved survival, bolstered $\mathrm{CD}^{+}$effector T-cell production and enhanced T-cell PDA-specific IFN $\gamma$ secretion within TME (129).

Significant progress has also been made against glioblastoma, commonly associated with extremely poor prognosis with $<2$ year median survival when treated with conventional chemotherapy (67). In contrast, PD-1/PD-L1 blockade following dendritic cell (DC) vaccination in a mouse model doubled the survival period (67). The fundamental mechanisms underlying this synergy were revealed as follows: (i) Glioblastoma requires substantial CD8 + NK cell infiltration; (ii) DC vaccination makes brain tumors permissive to T-cell infiltration; and (iii) subsequent PD-1 blockade neutralizes the suppressive checkpoint "shield" that would render T-cells dysfunctional (67).

\section{Checkpoint Blockers With ACT}

Adoptive-T-cell therapy aims to stimulate durable anti-tumor immune activity via (i) manipulation of T-cells ex vivo-TIL selection and expansion from patients before reinfusion-and (ii) gene therapy via sTCR (synthetic T-cell receptor) or CAR (chimeric antigen receptor) transfer into T-cells $(130,131)$. Checkpoint blockade prevents T-cell inhibition, as required by adoptive-T-cells for maximum anti-tumor activity, whilst its efficacy relies upon tumor-specific adoptive T-cells. So far, promising results have been obtained with CD19-specific CAR T-cell therapies, most notably against ALL, producing $90 \%$ total remission of which $67 \%$ showed sustained response after 6 months (132). High efficacy was also reported against advanced B-cell lymphoma (up to $53 \%$ complete response) (133).

The first FDA-approved CAR-T therapy Kymriah $^{\mathrm{TM}}$ (Tisagenlecleucel, CTL019) displayed durable remission in $83 \%$ of pediatric and young adult B-ALL patients tested (134). Also, CAR-T therapeutic Yescarta ${ }^{\mathrm{TM}}$ (axicabtagene ciloleucel, CT019) won FDA-approval following the success against refractory aggressive NHL with $82 \%$ ORR, 54\% CR, and $80 \%$ survival after 6 months. Neutropenia and leukopenia were the most frequently observed IrAEs but could readily be managed $(135,136)$. Furthermore, an ongoing trial progressing into phase II, tested anti-BCMA (B-cell maturation antigen) CAR-T therapy in late-stage relapsed/refractory multiple myeloma patients (NCT02658929) (137). Impressive 94\% ORR, and $56 \%$ CR rates were reported. Importantly, $90 \%$ of patients were given "minimal residual disease" (MRD) negative status, indicating an absence of even very small residual malignant cells, often remaining during or after remission, and responsible for relapse (137). Consequently, such studies suggest potential for CAR-T therapies to move beyond lymphoma and leukemia, and toward other pressing hematological cancers such as multiple myeloma (137). In contrast, unfortunately, ACT therapies have proven ineffective against solid tumors, often inducing life-threatening side effects such as CRS and respiratory distress (138). Nevertheless, preclinical tests using transgenic Her2 murine models of breast cancer have given promising results: Addition of a PD-1 inhibitor boosted Her2-specific CAR T-cells function and proliferation, with enhanced regression, compared to CAR T-cell administration alone (65). A major priority for this 
type of combination, therefore, is to identify ideal, tumor-specific antigens as novel co-targets.

Efficacy of ACT for solid tumors is also marred by challenges in T-cell delivery. CAR-T cells only demonstrate optimal performance if the local environment is sufficiently nutrientrich and waste products are easily removable (138). Accordingly, Smith et al. laced biopolymer scaffolds with the immunestimulatory STING (stimulator of interferon genes) agonist cdGMP (cyclic di-guanosine monophosphate) and CAR-T cells (138). In addition to their primary function as a T-cell delivery vehicle, These bioscaffold implants release cd-GMP to convert tumors into self-renewing vaccine sites whereby destroyed tumor cells provide an antigen source that then launch a broader, second-wave antitumor response after CAR-T cell release (138). Thus, compared with conventional systemic T-cell injections, significantly improved tumor regression, without significant toxicity, was obtained in mice against both inoperable pancreatic cancer and melanoma (138). Future trials involving biopolymer platform may incorporate, in addition to CAR-T cells, checkpoint blockers and IDO inhibitors (138).

CAR-T cells were recently succesfully generated in the mouse bloodstream in vivo in large quantities and with high efficiency $(139,140)$. Nanoparticles (NPs) possessing surface antiCD3e $\mathrm{F}\left(\mathrm{ab}^{\prime}\right) 2$ fragments and microtubule-associated and nuclear localization sequences were intravenously delivered, enabling NP cargo delivery to leukemia-specific T-cell nuclei (139). NPs contained a DNA plasmid encoding a CAR-construct flanked by transposon elements, which was incorporated into the Tcell nuclei genome via a piggyBac transposase-mediated cutand-paste mechanism (139). This technique circumvented the need for the expensive, overly elaborate and time-consuming $e x$ vivo manipulation of CAR-T cells required by current CAR-T generation approaches $(139,140)$. Consequently, combinations of nanocarriers + CAR-T cells, perhaps synergized with checkpoint blockade, could enable efficacious clinical translation of CAR-T therapies in the future.

\section{Checkpoint Blockers With Nanoparticles}

In a more recent approach, immunogenic "nano-scale coordination polymer" (NCP) particles, composed of oxaliplatin prodrug cores enclosed by a photosensitizer pyrolipid surface, were used to deliver chemotherapy and photodynamic therapy (PDT), respectively, to colorectal cancer in combination with anti-PD-L1 checkpoint inhibitors (74). NCPs represent a novel class of multimodality delivering self-assembled nanomaterials with a flexible composition that are biodegradable in host tissues. The “NCP@pyrolipid” hybrid nanostructure combines oxygen, light, and photosensitizers to generate unstable reactive ${ }^{1} \mathrm{O}_{2}$ species that can destroy target tumors by promoting apoptosis and acute inflammation. Oxaliplatin was shown previously to induce immunogenic cell death (ICD) against colorectal cancer (74). Thus, a three-way synergy with (i) pyrolipid-induced PDT, (ii) oxaliplatin chemotherapy, and (iii) checkpoint inhibition was reported. In murine colorectal cancer models treated with an anti-PD-L1 (pembrolizumab) and NCP@pyrolipid combination, CD8+ T-cell densities in tumors increased by up to 10-fold (74). Furthermore, addition of localized PDT induced an abscopal effect: targeted tumors shrunk by $67 \%$ while distant tumors regressed almost completely. This "triple combination" may also be applicable to other metastatic cancers with PDT-accessible primary tumors (74). This was indeed confirmed in murine models for a combination of anti-PD-L1 (pembrolizumab) + ZnP@pyro + PDT against both primary and metastatic breast cancer (75). Nanoparticles that spatio-temporally delivered antiPD-1 checkpoint inhibitors and agonistic anti-OX40 antibodies simultaneously to mouse $4 \mathrm{~T} 1$ breast cancer significantly elevated T-cell stimulation via enhanced release of IFN- $\gamma$ and increased CD8+:Treg cell ratio, resulting in doubling of survival rates (141).

On the other hand, development of immunomodulatory nanoparticle-based vaccines has been constrained by the tendency of phagocytes to sequester nanoparticles, blocking their access to target and leading to harmful accumulation in spleen and liver (142). Luo et al. have reported a versatile nanovaccine platform, in which a synthetic polymeric nanoparticle PC7A enhances cross-presentation of antigens, transports antigens to lymph nodes and functions as an immunogenic, tumorsuppressive adjuvant by activating "Stimulator of Interferon Genes" (STING) pathways (142). Consequently, upon ingesting PC7A, phagocytes are reprogrammed from "foe to friend." The nanovaccine inhibited colon and melanoma tumor proliferation in preclinical mouse models (143). In the mouse TC-1 tumor model, synergy with PD-L1 blockade produced 100\% survival even after 60 days, implying robust anti-tumor memory (142, 143).

Some success with SYMPHONY ("Synergistic Immuno Photodermal Nanotherapy"), a novel combination of gold nanostars (GNS), laser light and PDL1 blockade has been reported (76). Gold nanostars preferentially accumulate inside tumor cells due to their sharp spiked geometry, functioning as "lightning rods" that efficiently capture and convert laser light energy into heat, triggering thermic death of tumor cells deep within malignant tissues (76). Thus, SYMPHONY demonstrated significant superiority over anti-PDL1 monotherapy for both primary and distant metastatic bladder tumors (76).

\section{Oncolytic Viruses}

Oncolytic viruses aim to specifically infect tumors, then replicate within and destroy them, sparing healthy cells (144). However, whilst such therapy can also induce immune-based anti-tumor responses, host immunity can limit the spread and replication of OVs (144). Since checkpoint blockade is most effective in patients harboring immunogenic tumors, OVs are ideal combination candidates given their ability to induce TME immunogenicity by developing optimal conditions for T-cell priming and activation (145). Additionally, OVs have favorable safety profiles, with only mild flu-like symptoms reported (144). A modified herpes simplex virus taliogene laherparepvec (TVEC) was FDA-approved after showing durable responses in 16\% of resectable melanoma patients in phase III trials (146). More recently, CTLA- 4 blockade was combined with CAVTAK ${ }^{\mathrm{TM}}$, an immunotherapeutic and oncolytic strain of Coxsackievirus A21 (CVA21), itself an unmodified common cold RNA virus (147). In phase Ib trials on advanced melanoma, impressive $60 \%$ 
response rates and $78 \%$ disease control rates were obtained with only $8 \%$ irAEs (NCT02307149, ongoing) (69). In mouse models of aggressive ovarian and colon cancers, combination of $\mathrm{OV}$ expressing CXCL-11 (C-X-C motif of chemokine 11 precursor) with PD-L1 blockade markedly increased PD-L1 expression in TMEs, eliminated MDSC, Treg, and TAM immunosuppressive cells, and boosted T-cell infiltration, leading to complete responses in $40 \%$ of cases (148).

A pressing issue facing immune-oncology is converting immunologically "cold" and unresponsive tumors into more therapeutically receptive "hot" tumors, characterized by high CD ${ }^{+}$TIL infiltrate, PD-L1 expression and mutational load (149). Ribas et al. reported a promising phase Ib trial, where intralesional combination of talimogene laherparepvec (T-VEC) with PD-1 blockade induced $62 \%$ response rates (33\% complete) in patients with advanced metastatic melanoma (66). Further work is needed to understand the mechanistic basis of this synergy (66).

As regards brain tumors, traditionally, OVs are injected intralesionally because of the blood-brain-barrier (150). However, a naturally-occurring human oncolytic Orthoreovirus was intravenously administered to patients prior to having their glioma tumors surgically removed within days. Resected tumors were then examined and were found to contain low quantities of viral capsid proteins in $100 \%$ of patients, indicating successful reovirus penetration into the brain (150). Minor side-effects of low-grade lymphopenia and minor flu-like symptoms were reported. Tumor PD-L1 expression is a major determinant of PD-1 inhibitor efficacy, and occurs at relatively low levels in glioblastomas (150). Critically, PD-L1 expression was significantly elevated in tumor extracts from reovirus-treated patients compared to controls, providing a rationale for synergistic combination with PD-1 blockade $(151,152)$. In a subsequent combination study on a immunocompetent orthotopic murine glioma model, sequential administration of reovirus for 2 weeks followed by PD-1 inhibitors for 1 week showed $100 \%$ survival up to 48 days, compared with monotherapies (36 days for reovirus and 22 days for PD-1 blockade (150). Furthermore, reovirus bolstered TIL recruitment, with $20 \%$ of total tumor cells containing $\mathrm{CD}^{+}$TILs in reovirus-treated mice vs. $2 \%$ in controls (150). Similarly, Maraba virus + PD-1 blockade in a murine model of breast cancer showed improved TIL infiltration, PD-L1 upregulation and survival relative to monotherapies (153).

Most recently, the Zika virus (ZIKV), well known to cause microcephaly and miscarriages in pregnant women across South America, has demonstrated significant oncolytic activity against glioblastoma in murine models (154). This was made possible by the ability of ZIKV to penetrate the blood-brain-barrier. Thus, Zhu et al. showed that mice treated with mouse-adapted ZIKV had significantly improved life-span (50\% survival after 63 days vs. no survival after 30 days in the control group), with negligible effect on healthy neurones (154). Genetically engineered ZIKV strains could further improve safety, and future trials in combination with checkpoint blockade would seem promising (154).

\section{Synthetic Biology: "Gene Circuits"}

Major limitations of cancer immunotherapies include scarcity of tumor-specific antigens, direct immunosuppression by tumors and "off-target" toxicity (155). Recently, an immunomodulatory synthetic RNA-based "circuit" (two artificial tumor-specific promoters integrated with an RNA-based AND gate mechanism) was delivered by lentivirus into ovarian tumor cells to overcome such issues. Upon activation of both promoters exclusively within specific cancer cells, the AND gate expressed GAD fusion proteins to drive co-expression of a suite of immunomodulators termed "SCIP" comprising: (i) surface T-cell engagers (STE) and chemokines (CCL21) to label tumors for T-cell mediated destruction; (ii) IL12 cytokines to boost T-cell activation and effector activity; and (iii) PD-1 checkpoint blockade (155). In human ovarian cancer murine models, this approach resulted in stable SCIP-producing gene circuits, leading to unprecedented reduction in tumors to untraceable levels, and an OS level of 80 vs. $0 \%$ for controls. This effect was robust even when only 15$30 \%$ of the target tumor cells expressed SCIP. Such "gene circuits" can be expected to be applied to diverse malignancies (155).

\section{POTENTIAL, NOVEL IMMUNOTHERAPY CO-TARGETS}

In this section, we highlight a number of less widely recognized, emerging mechanisms that could potentially serve as co-targets in combination immunotherapy.

\section{Metabolic Components}

A number of metabolic mechanisms have been shown to be essential for immune evasion of tumors and could serve as co-targets in immunotherapy (15). Tumors demand an expansive, adaptable metabolic framework to thrive in specific niches, and all contemporary cancer hallmarks require metabolic engagement to some degree $(156,157)$. Recent evidence suggests that tumors may perpetuate their survival by reprogramming host metabolism (158). In patients with both anorexia and tumors, the increased metabolic stress causes elevation in systemic glucocorticoid hormones that alone can significantly decrease antitumor T-cell immune response, cause tumor growth and self-perpetuate the cycle (158). Novel combination approaches should therefore aim to normalize metabolic stress in parallel with checkpoint blockade to optimize clinical outcome. Notable metabolic targets of therapeutic interest include the tryptophan catabolizing enzyme indoleamine 2,3-dioxygenase (IDO), Notch homolog 1 (NOTCH1), and cyclooxygenase-2 (COX2) (159-162).

Migration of immunosuppressive Treg cells to inflamed malignant tissues relies upon glucokinase-mediated glycolysis. Glycolysis is initiated by glucokinase (GCK), itself induced via the P13K-mTORC2 signaling pathway (163). Treg cells lacking components of this pathway remain immunosuppressive. Patients possessing a polymorphism causing elevated GCK activity saw enhanced Treg cell motility, given that GCK promotes cytoskeletal restructuring via actin association (163). Consequently, there exists potential for inhibition of glycolytic 
enzymes to manipulate the migration capacity of T-cell subsets, and thus to "soften" the immunosuppressive role of the TME (163).

Aerobic glycolysis, characteristic of growing tumors, fuels optimal T-cell effector function (159). In highly antigenic regressive tumors, competition for glucose in TME was found to be sufficient alone to drive cancer progression (159). This would occur as tumors surpass T-cells for glucose, directly suboptimizing T-cell function by impeding their IFN $\gamma$ production, critical for anti-tumor activity. Combination strategies that couple the depletion of tumorigenic immune cells with glycolysis enhancement in infiltrating T-cells, therefore, may prove effective at metabolic remodeling of the TME (159). This could also explain why combined anti-CTLA- $4+$ anti-PD-1 checkpoint blockade is particularly effective since anti-CTLA-4 would deplete Tregs whilst anti-PD-1 would directly dampen tumor glycolysis by inhibiting the mTOR pathway (159).

Drugs targeting tumor metabolism are in early trials. COX2 is essential for the production of the tumor-sustaining mediator prostaglandin $\mathrm{E} 2\left(\mathrm{PGE}_{2}\right)$, a prostanoid lipid that enhances cancer survival, metastasis, and immunosuppression (162). COX2 is overexpressed in several cancers, and COX2 inhibitors (e.g., aspirin) were found to act synergistically with PD-1 blockade in preclinical trials against melanoma, breast and gastric cancers $(162,164)$. COX2 inhibition would render melanoma and breast cancers vulnerable to immune control, restoring tumor immunosurveillance via CD8 $+\mathrm{T}$ and $\mathrm{NK}$ cell recruitment and promotion of the anti-tumor M1 macrophage phenotype (162, 164).

A recent metabolomics approach implicated CSCs in cancer metabolism. Chronic myeloid leukemia (CML) stem cells were found to be maintained by dipeptides accumulated via upregulated expression of Slc15A2 dipeptide transporters (165). In vivo, the internalized dipeptides would activate amino acid signaling via the p38MAPK-Smad3 pathway to sustain CML stem cell activity. Furthermore, TKIs could be synergized with the dipeptide transporter inhibitors. Such synergies showed highly specific responses in CML-affected mice where normal stem cells were insensitive to transport inhibitors (165). These observations would suggest that TKIs plus inhibitors of metabolic nutrient signaling within the p38MAPK-Smad3 pathway (e.g., Tocriset ${ }^{\mathrm{TM}}$ and SIS3) could be beneficial to CML patients (Supplementary Figure 2) (165). Additionally, CSCs indirectly dampen antitumor immunity via secretion of the immunosuppressive factors TGF- $\beta$ and arginase (to promote inhibitory Treg and TAMs) and attenuation of STAT-3 and PDL1 surface proteins to inhibit antitumor CD8+ T-cells (166). Thus, a two-pronged therapeutic approach may be possible, where an initial TKI/anti-dipeptide transporter combination clears CSCs-a major component of tumor recurrence-and then an anti-PD-1 checkpoint inhibitor is added.

Metabolomics has also identified glutaminase as a potential target downstream of NOTCH1 (161). The latter is a conserved single-pass transmembrane receptor, crucial to T-cell lineage commitment and itself a major T-ALL target, given that activating NOTCH1 mutants are common in T-ALL (161). Glutaminolysis was identified as an integral pathway for leukemia cell proliferation controlled by NOTCH1 and, therefore, a critical determinant of anti-NOTCH1 clinical efficacy. Consequently, glutaminase + NOTCH1 inhibitor combinations showed a potent synergistic anti-leukemic effect in vitro in patient-derived T-ALL murine models (161) (Supplementary Figure 3).

In conclusion, metabolomics promises to identify novel therapeutic targets and mechanistic insights, and may provide important links reflecting the role of genetic, microbiome, lifestyle, and environmental factors in tumor development (167).

\section{Exosomes}

Exosomes are specialized, nano-sized lipid bilayer vesicles that enable a novel means for intercellular communication, shuttling bioactive DNA, mRNA, miRNA, and oncogenic proteins between cells, thereby enabling genetic reprogramming of cellular networks (168). Various stages of the cancer process involve exosomal interactions (Figure 7). Thus, exosomes transmit messages from tumor cells to both stromal and immune cells, facilitating immune evasion, and establishment of the tumor niche (168). Exosomes may be therapeutically exploited via three approaches, as follows:

i. Direct exosome-based immunotherapy. This is exemplified by "dexosomes" (dendritic cell-derived exosomes) loaded with whole antigen or peptide fragments, and have proven ability to induce systemic T-cell responses (169). Immunostimulatory dexosomes are especially promising, stimulating antitumor responses with greater accuracy than possible using noncellular approaches, and possessing higher biostability and bioavailability as well as cost-effectiveness compared with other cellular therapies (169). Treatment of human breast cancer with dexosomes resulted in incorporation into tumors and subsequent expression of dexosome immunostimulatory molecules (e.g., CD86, CD81, MHCI/II + tumor antigen) on tumor cell surfaces, thus boosting tumor immunogenicity and T-cell engagement (170). Dexosome-treated tumors indeed contained a much higher proportion of T-cells secreting IFN- $\gamma$ immunostimulatory cytokines (170). However, early clinical trials on colorectal and NSCLC have yielded only moderate efficacies (171). Efficacy may be improved by better composition/antigen-loading strategies and trafficking of exosomes $(171,172)$.

ii. Exosome elimination in patients with advanced cancer. This represents a new treatment concept, demonstrated for the blood-pressure dampening drug, amiloride. This decreased MDSC immunosuppressor functions in colorectal cancer patients by inhibiting exosome formation (168).

iii. Exosomes as "natural nanoparticle" drug delivery vehicles. As such, exosomes exhibit favorable biocompatibility and biodistribution (173). Indeed, use of macrophage-derived exosomes to transport paclitaxel into multidrug resistant (MDR) tumors enhanced treatment efficacy by 50 -fold relative to paclitaxel administration without exosomes (173).

In conclusion, given their diverse roles in facilitating both tumorigenesis and immunosuppression, tumor-derived exosomes (TEXs) are attracting increasing interest as therapeutic 


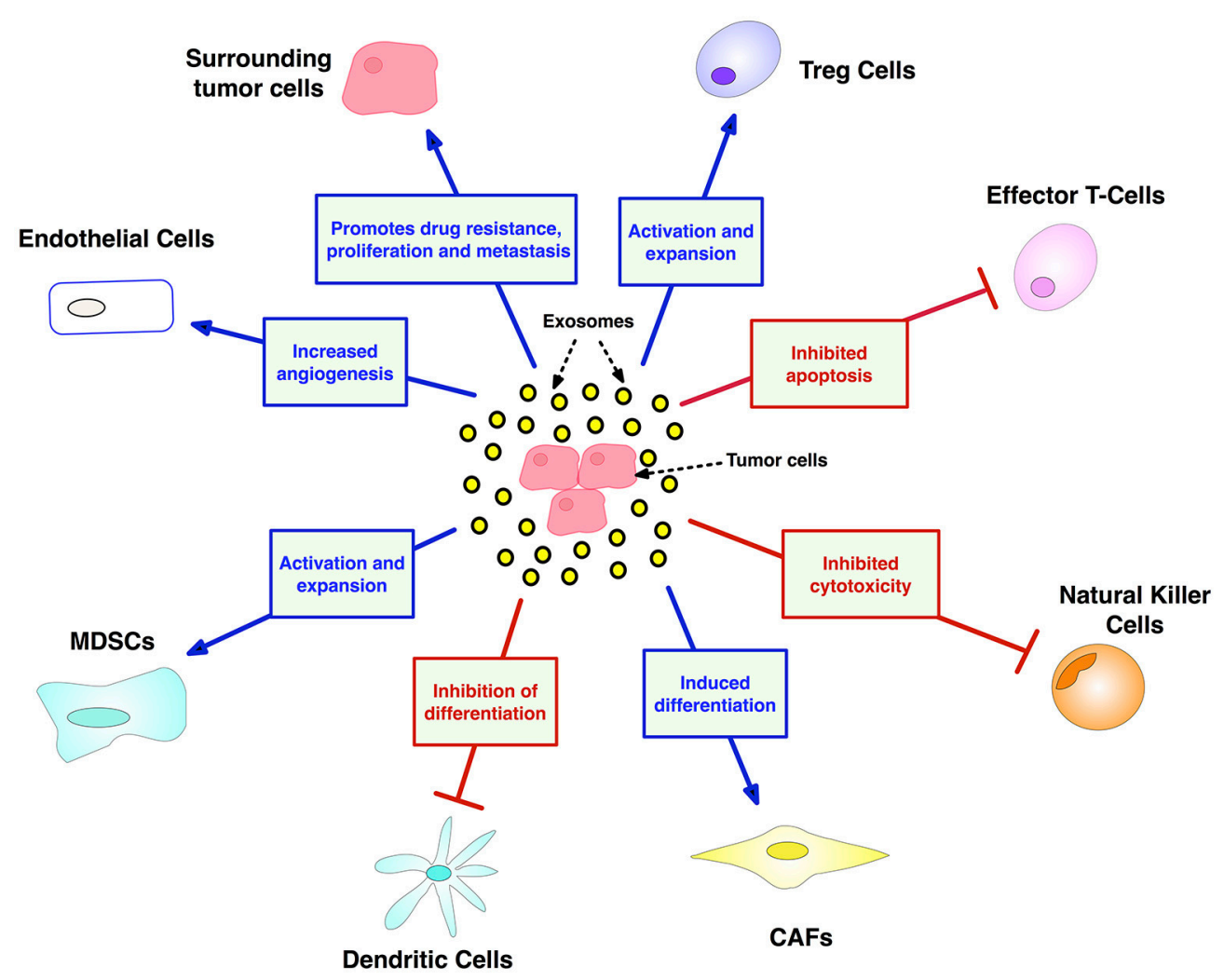

FIGURE 7 | Exosome contributions to cancer facilitated by transport of oncogenic nucleic acids and proteins. Exosomes have major and diverse roles in tumorigenesis, including: (i) promoting an immunosuppressive TME by dampening NK and T-cells, while expanding inhibitory Treg and MDSC populations, (ii) mobilizing neutrophils, and thus skewing marcophages toward their M2 immunosuppressive form (iii) maintaining tumor drug resistance by exporting antitumor drugs and shuttling multi-drug-resistant proteins (iv) support tumor thrombosis and angiogenesis by activating endothelial cells (v) promoting metastasis by converting fibroblasts into myofibroblasts. CAF, cancer-associated fibroblasts; MDSC, myeloid-derived suppressor cell. Created using information from Zhang et al. (168).

targets (174). Recently, genetically modified K562 leukemiaderived exosomes expressing IL-15, IL-18, and 4-1BBL surface proteins displayed a biphasic effect upon human NK cells (i) enhancing cytoxicity and proliferation (4h) and (ii) inhibiting activated receptor expression and dampening cytotoxicity (48 h) (175). These results raise the possibility of using TEXs as anti-tumor vaccines (175). More data is needed, however, to assess the clinical potential and safety of exosomes as monoand/or combination-targets (168).

\section{Dividing T-Lymphocytes}

The asymmetrical division of T-cells observed in murine models represents a novel opportunity as an unconventional immunotherapy target (176). When a "mother" T-lymphocyte naive to immune stimulation undergoes mitosis, mTORC1 (an enzyme responsible for protein synthesis) is divided unevenly between the two daughter cells. The progeny with the higher mTORC1 becomes strongly activated as a potent killer Tcell whilst the "sister" cell displays behavioral traits more associated with memory T-cells (176). This raises the possibility of exploiting mTORC1-expressing T-cells as a target for longterm potentiation of immunotherapy, by skewing development toward memory T-cells (176). Proteasome-activators such as
Cyclosporine were found to tip the balance of dividing CD8 ${ }^{+}$ $\mathrm{T}$-cell progeny toward memory $\mathrm{T}$-cells (exploiting the fact that effector and memory T-cells have differing proteasome activity levels) (177). Thus, there exists potential for synergizing immunotherapy with proteasome modulators (177).

\section{Ion Channels}

A variety of ion channels, including voltage- and ligand-gated ion channels, are expressed in cells of the immune system and make significant, dynamic contributions to immune functioning (178). Here, we highlight voltage-gated sodium channels (VGSCs/Nav's), voltage-gated potassium channels (VGPCs/Kv's), and calcium-activated potassium channels ( $\mathrm{K}_{\mathrm{Ca}}$ 's).

VGSCs may manifest themselves in immuno-oncology and serve immunotherapy in several different ways. First, Nav1.5 was shown to control the positive selection of $\mathrm{CD} 4+\mathrm{T}$ cells from $\mathrm{CD} 4+/ \mathrm{CD} 8+$ thymocytes in response to stimulation by APCs $(179,180)$. The selected cells would play a central role in immune functioning via production of cytokines and chemokines, facilitating antibody production by B-cells, maintaining immunological memory and priming CD8+ CTLs. Consequently, VGSC blockers could reduce the CD4+:CD8+ ratio, thus boosting $\mathrm{CD} 8+\mathrm{CTL}$ populations that drive early 
immunosurveillance antitumor responses. Furthermore, high CD8+ TIL content of tumors is predictive of pathological complete response to primary systemic therapy regardless of cancer subtype (181). However, as a monotherapy, VGSC blockers may yield only short-term success since depleted CD4+ T-cells would ultimately reduce immunological memory and compromise CTL tumor re-challenge (182). Accordingly, tumor vaccine delivery after VGSC inhibition as a sequential "onetwo punch" could activate new thymic CD4+ helper T-cells to restore lost immunological memory and sustain efficacious CTL antitumor responses (182). Additionally, VGSC blockers would increase tumor "hotness" by enhancing CTL presence, and thus synergize with PD-1 blockade (149). Second, functional VGSC expression occurs in macrophages, another cell type in the innate immune system. When recruited to tumors, macrophages can accelerate cancer progression. A recent study by RohJohnson et al. on zebrafish and mouse models of melanoma showed that recruited macrophages transferred their cytoplasm into melanoma cells and this promoted metastasis (183). How such intracellular communication is regulated and the nature of the transferred molecules were not known (183). Interestingly, however, VGSC activity drives macrophage motility (184). Accordingly, VGSC blockade could eliminate this component of immune response and could form the basis of mono- and/or combination immunotherapy with tumor vaccines or with PD1 blockade to dampen TME immunosuppression, overcome PD-1 resistance and enhance patient responses (149). Third, the predominant VGSC in cancers of breast and colon is the neonatal splice variant of Nav1.5 (nNav1.5) $(185,186)$. This offers several advantages as a target, including having an extracellular region that can be targeted by an antibody and a restricted expression pattern in the adult human body (187, 188). Accordingly, nNav1.5 exprssion may be cancerspecific and could form the basis of CAR-T immunotherapy. Importantly, in all such cases, VGSC blockers would additionally suppress the invasiveness of the tumor cells themselves (189, 190).

The role of Kv1.3 channels in the immune responses to tumors may be more complex, dependant dynamically on disease stage (191). On the one hand, tumor infiltration may involve downregulation of the channel (192). On the other hand, $\mathrm{Kv1} .3$ (and $\mathrm{K}_{\mathrm{Ca}} 3.1$ ) channels are expressed predominantly in CD8+ T-cells and contribute to membrane electrogenesis and calcium influx, crucial to their antitumor granzyme B and cytokine production (193). Kv1.3 activity also promotes T-cell proliferation and high-level expression of Kv1.3 correlated with elevated levels of Ki-67 (193). Finally, a novel novel role for Kv1.3 has been proposed in TME where cell death within a necrotic region can release cellular $\mathrm{K}^{+}$into the extracellular spaces (194). Exposure of T-cells to such high $\mathrm{K}^{+}$can suppress their activation and functioning by increasing intracellular $\mathrm{K}^{+}$and inhibiting PP2A-dependent/TCR-activated Akt-mTOR signaling (194). Accordingly overexpression of Kv1.3 restored antitumor T-cell functionality by facilitating efflux of the high intracellular $\mathrm{K}^{+}$, leading to enhanced survival of tumor-bearing mice (194). Overall, therefore, Kv1.3 expression in T-cells can promote the immune reaction to tumors once the cells enter TME.
Calcium-activated potassium $\mathrm{K}_{\mathrm{Ca}} 3.1$ channels are upregulated in activated T-cells and also play a significant role in regulating cellular migration and proliferation (195). Upon activation by tumor cells, adherent NK (A-NK) cells preferentially upregulated $\mathrm{K}_{\mathrm{Ca}} 3.1$ channels (196). Blocking $\mathrm{K}_{\mathrm{Ca}} 3.1$ activity with TRAM-34 increased the degranulation and cytotoxicity of ANK cells, and induced increased ability of A-NK cells to reduce tumor growth in vivo. Taken together, these results rationalize the co-targeting of $\mathrm{K}_{\mathrm{Ca}} 3.1$ and $\mathrm{PD}-1$ on NK cells in future cancer immunotherapy $(197,198)$. NK cells suppress metastasis by inducing degranulation-mediated tumor cell lysis via release of perforins and cytotoxic granzymes. $\mathrm{K}_{\mathrm{Ca}} 3.1$ blockers TRAM34 and NS6180 increased NK cell proliferation and enhanced degranulation rate of the non-adherent K562 erythroleukemia cells in vitro (199). On the other hand, Kv1.3 blockers Stichodactyla toxin (ShK) and 5-(4-phenooxybutoxy) psoralen (PAP1) decreased proliferation and degranulation, consistent with Kv1.3 being essential to NK-induced cytotoxicity (199).

In conclusion, VGSC (in particular, nNav1.5), Kv1.3, $\mathrm{K}_{\mathrm{Ca}} 3.1$, and probably other ion channels and transporters represent novel immunotherapy targets. Importantly, since ion channels are also involved at all stages of the overall cancer process, their blockers may offer unique multi-faceted advantages for Tcell based immunotherapies, including combinations with PD1 blockade (196, 198, 200) (Figure 8). A further advantage of ion channels is their ability to be manipulated remotely using optogenetics techniques (202).

\section{PERSONALIZATION OF IMMUNOTHERAPY}

Checkpoint inhibitors can be applied to a wide spectrum of solid tumors and hematological cancers (132). This is possible given the conserved upregulation of PD-1 and other immune pathways across a plethora of different human cancers (203). However, PD-1 blocking drugs do not demonstrate efficacy against the full range of malignancies and not all patients with PD- $1^{+}{ }^{+}$tumors yield a response. This has been attributed to the phenomena of "mixed tumor regression" in which malignant growths at different locations within the body (even within a given tumor) of a patient display variable therapeutic responses (203). Thus, currently, only a limited subpopulation of patients can benefit (12). In order to enhance treatment efficacy, reliable biomarkers-ideally several screened simultaneously in multiplexed assays-are essential to identify patients likely to give the best response to a given immunotherapy regimen with minimal toxicity (203) (Figure 9A). CD8+ density, mutational load and PD-L1 expression as solitary biomarkers are not sufficient to effectively characterize the TME given their complex interdependency (205). Tumor mutational burden (TMB) indicates the total number of somatic mutations/ $\mathrm{Mb}$ of DNA, where high TMB tumors (melanoma and NSCLC) are more likely than low TMB tumors (ovarian and breast carcinomas) to harbor foreign neoantigen proteins, resulting from these mutations (206). Checkpoint blockade can then stimulate and enable host immunity to detect neoantigens and destroy the tumor, demonstrating greater efficacies against higher 


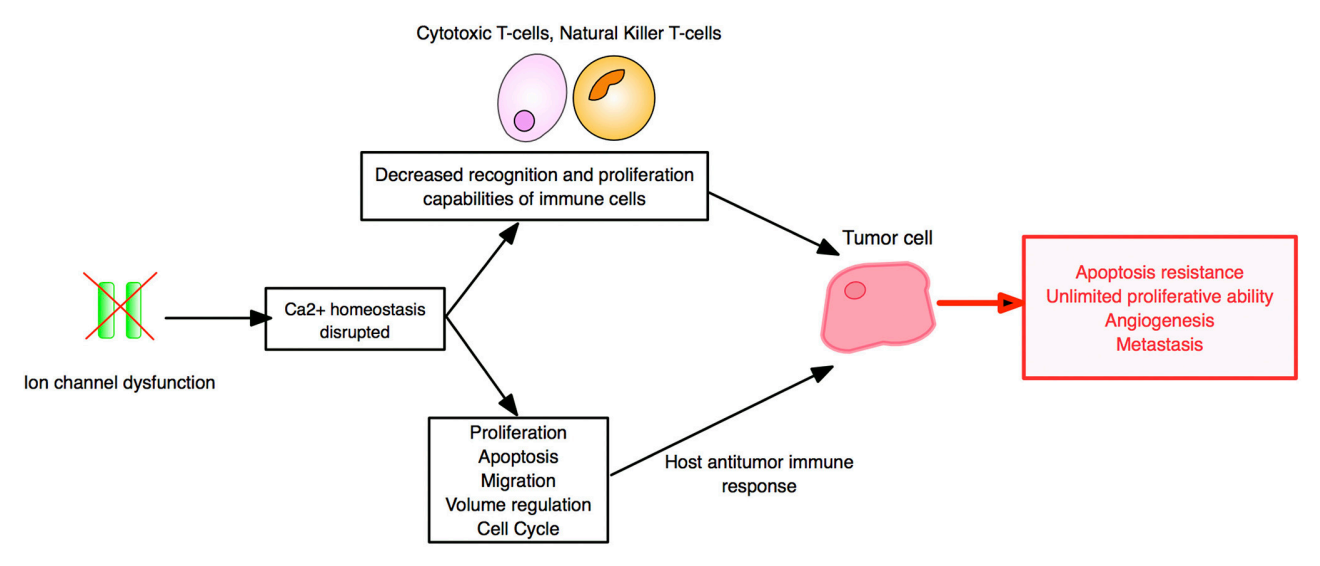

FIGURE 8 | lon channels as cancer immunotherapy targets. The impact of ion channel dysregulation upon tumor-immune system interactions is depicted. In both immune and tumor cells, ion channels are involved in regulating $\mathrm{Ca}^{2+}$ influx and downstream signaling pathways. Dysregulation of ion channels can directly fuel carcinogenesis, and immune cell cytotoxicity is dampened by alterations in $\mathrm{Ca}^{2+}$ signaling. Cancer hallmarks are boxed in red. Created using information from Bose et al. (200), Litan and Langhans (201), and Panyi et al. (193).

TMB tumors (206). While TMB calculation is necessary, alone it is not indicative of checkpoint blockade response and therefore of limited clinical use (207). However, it could serve as a component of an integrated algorithm (also involving host genetics, microsatellite instability, neoantigen load, TIL content, the TME, and microbiome) to accurately determine patient response to immunotherapy $(207,208)$. Effective biomarker quantification is also essential for early detection, functional diagnosis and monitoring of treatment efficacy/disease progression (209). In these regards, novel biomarker-driven surrogate endpoints of PFS and ORR should be identified in order to earlier identify clinically meaningful responses (210-212).

Lesterhuis et al. proposed that effective therapeutic response to immune checkpoint blockade should follow a "critical state transition" comprising the following: (i) an initial stable state (where static, pre-treatment biomarkers are obtained at a single time point); (ii) a pre-transition state (following treatment initiation, from where the system can still revert back to the stable state); (iii) a critical transition state (reached when a biological "tipping point" or a small change to the system from checkpoint blockade occurs); and (iv) a new stable state (where malignant tissue reverts back to healthy tissue) (213). Emerging dynamic biomarkers, associated with treatment response, can be obtained from biopsies at multiple time intervals comparatively in nonresponding and responding patients. Importantly, a "network biology" approach might identify dynamic biomarker "warning signals" near the "tipping point" by mapping molecular changes associated with tumor regression after checkpoint inhibitor therapy (213). This approach can potentially identify "gene hub products," driving response, that could serve both as dynamic biomarkers and novel drug targets for combination therapy. Targeting such hubs can significantly increase both the proportion of patients benefiting from treatment as well as the overall magnitude of responses to checkpoint blockade (213).

Khalil et al. discussed the potential for personalized immunotherapy based upon tumor phenotype (15). Checkpoint immunomodulators are predicted to yield the best therapeutic response in tumors harboring a high neoantigen or mutational burden, immunosuppressive TME and high density of TILs, ideal for melanomas and lung adenocarcinomas (4, 15). CAR T-cell therapy would be optimal against leukemias and medulloblastomas that possess immune-permissive TMEs and lower antigenic diversity (15). This strategy can be used to design hybrid combinations of CAR T-cell therapy and checkpoint blockade that might address tumors with intermediate phenotypes, such as myelomas, and cancers of prostate, ovary, kidney, and liver. Heterogeneity of TMEs can also be characterized by single-cell flow cytometry analysis so as to evaluate possible inherent immune evasion mechanisms (214). Tumors can thus be stratified by phenotype to enable appropriate personalized treatment (Figure 9B) (5).

As already emphasized, the "immune contexture" (i.e., spatial organization, density and composition of the tumor immune infiltrate and function) directly influences cancer progression (215). For example, CD8+ T-cell density correlates with good prognoses in breast, colorectal and head and neck cancers, but poor for RCC. The influence of tertiary lymphoid structures is beneficial for pancreatic and breast cancer and NSCLC, yet negative for hepatocellular carcinoma. Treg cell abundance affects colorectal and gastric cancer prognoses positively, but is detrimental for breast, pancreatic cancers, and NSCLC (215). Decoding the immune (I), vascular (V), and stromal (S) components of TMEs via in situ immunophenotyping, immunohistochemistry and "omics" technologies revealed that checkpoint inhibitors perform optimally for tumors with high I, low V and low S content. CAR-T, vaccination and chemo- or radiotherapy are ideal for TMEs lacking any of these features, and combinations are suitable for malignancies with low I and high V and $S$ (215). Thus, uncovering other predictive biomarkers for immune blockade responses-such as CTLA-4/PD-1 expression on immune infiltrate and tumor cells, TILs, and circulating MDSCs and lymphocytes-should be prioritized in order to 


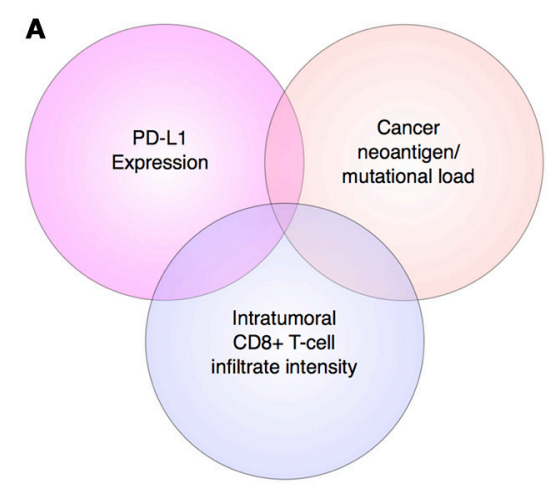

$\mathbf{B}$

FIGURE 9 | Personalized immunotherapy. (A) Multifactorial biomarker panels. The three most widely established biomarkers of response to anti-PD-L1 immunotherapies have strong functional overlap, hence all three will soon be used together to provide stronger predictive value of therapeutic outcome than single biomarkers. Adapted from Topalian et al. (203). (B) TME stratification into 4 categories based upon PD-L1 expression and presence of TILs (tumor infiltrating lymphocytes) by Teng et al. (204). This promises to enable prediction of patients that respond to checkpoint blockades, namely anti-PD-1 and anti-PD-L1 (204).

improve the efficacy of personalized treatments of patient (215, 216).

A multiplex immunohistochemical imaging system $\left(\mathrm{HALO}^{\mathrm{TM}}\right)$ has been devised that enables simultaneous use of five different stains and permits analysis of any organelle or subcellular compartment (217). Such a system would be ideal for immuno-oncology where several biomarkers are needed to characterize distinct tumor and immune cell populations within tumor biopsies. Specifically, $\mathrm{HALO}^{\mathrm{TM}}$ Proximity and Tumor Infiltration analyses allow precise quantitation of (i) the spatial relationship between the two cell populations and (ii) the position of immune cell density relative to the invasive tumor margin, respectively (217). In another technical approach, MultiOmyx multiplexed TIL panels yield comprehensive immunophenotypic profiling of tumors even from a single tissue section (218). Successful analyses of immune responses within solid TMEs have revealed two basic categories: high-TIL and low-TIL tumors (218). Such immunophenotypic analyses can facilitate personalized treatment regimens whereby high-TIL tumors would be treated effectively with checkpoint inhibitor monotherapies. In contrast, low-TIL tumors would respond most effectively to combination immunotherapies incorporating an agent that boosts the endogenous anti-tumor response (218).

Expression and (co)localization of as many as 12 biomarkers can be quantitatively and qualitatively analyzed in single biopsy sections (218). Increasingly, genetic mutations in tumors are being profiled as the bases of patient suitability for specific therapies $(219,220)$. Furthermore, it is possible to profile DNA mutations in "liquid biopsies" in the form of ctDNA (circulating tumor DNA) shed from tumor cells into blood (221). Such procedures have the additional advantages of being relatively non-invasive and potential to detect therapy resistance-promoting mutations not readily revealed in tissue biopsies and to enable monitoring of tumor progression over time (221). Ultimately, liquid biopsies may complement tissue biopsies to refine immunotherapies and may not replace tissue biopsies at least in the short-term $(222,223)$. Tumor biopsies can also be subject to parallel (whole-exome and RNA-based) sequencing to identify tumor-specific mutations and associated neoantigens which would normally enable the immune system to differentiate between host and malignant cells (Figure 10). Neoantigens represent potential biomarkers of immunological activity and have been used to design personalized synthetic DNA, RNA, or peptide vaccines that target patient-specific neoantigen spectra. The most immunogenic mutant peptide epitopes from in silico prediction algorithms can then be introduced into the vaccine formulation and combined with PD-1 and/or CTLA-4 checkpoint blockade (Figure 10). Such personalized therapies can be engineered to enhance neoantigenspecific T-cell activities, and could also expand the cancer types that can be treated by immunotherapy.

Searching "The Cancer Genome Atlas" (TCGA) with algorithms, such as domainXplorer, identified 122 potential cancer driver genes corresponding to mutational status of TIL proteins (226). Furthermore, TCRs can be provided by neoantigen-sensitive donor T-lymphocytes, and can be wielded to retarget naïve patient T-lymphocytes and re-engage tumors (4). Clinical response durability could be improved by simultaneously targeting several neoantigens homogenouslyexpressed in tumors (4). Such tools could ultimately generate a comprehensive archive of drivers of gene mutations modulating anti-tumor immunity and thus enable design of specific immunotherapies.

Whole-genome sequencing of individuals with adult T-cell leukemia unveiled a novel genetic mechanism tied to immune evasion involving structural variants of the $3^{\prime}$ terminal of PDL1 (227). Such variants, involving deletions, duplications, and other break points, would boost functional PD-L1 expression to abnormally high levels. Murine models with intact $3^{\prime} \mathrm{PD}-\mathrm{L} 1$ termini showed both enhancement of T-cell proliferation within the lymphoma TME and tumor regression (227). This would suggest that the $3^{\prime} \mathrm{PD}-\mathrm{L} 1$ terminal could serve as a diagnostic biomarker enabling selection of patients that would best respond to PD-L1 blockade therapies (227). 


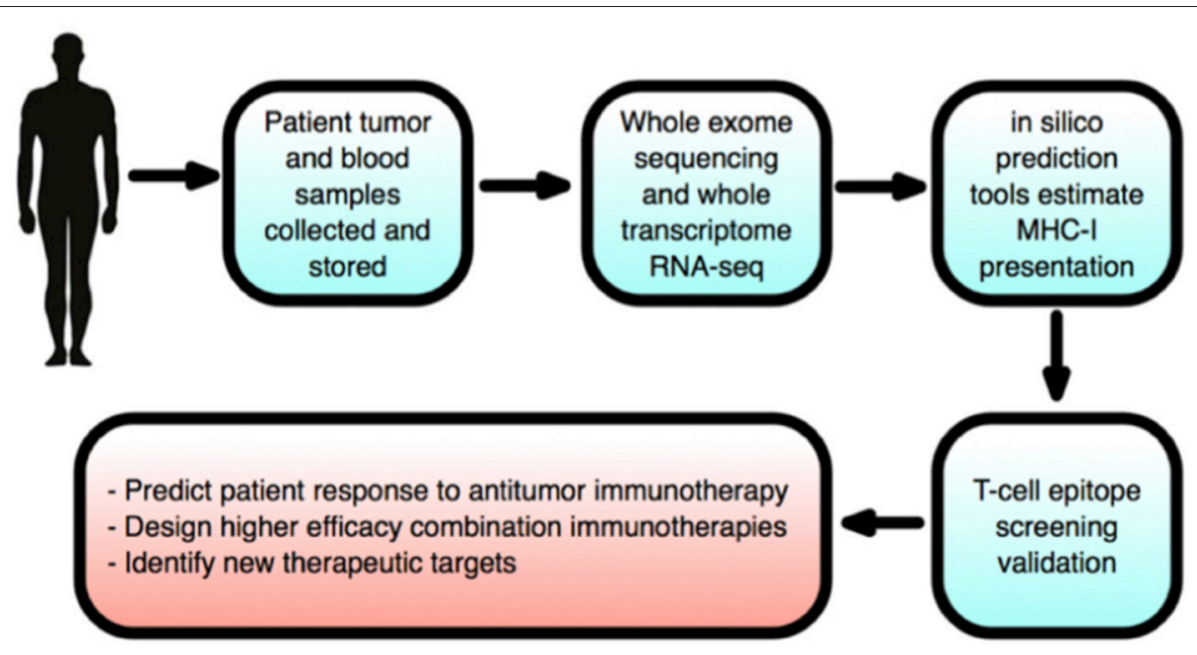

FIGURE 10 | New neoantigen discovery pipeline to facilitate personalized immunotherapy. Neoantigens are mutated antigens that are unique to cancer cells, foreign to the immune system and severely limit immunotherapy efficacy. Checkpoint inhibitors stimulate and enable host immunity to detect neoantigens and destroy tumors. Following collection of patient tumor and blood samples, whole exosome sequencing on malignant and non-transformed cells derived from the same patient reveal tumor-specific mutations. Subsequently, whole-transcriptome RNA-sequencing establishes mutation expression levels, before in silico tools can identify neoepitopes (tumor-specific mutation-derived peptides presented on tumor cells via the MHC-I and recognized by T-lymphocytes). To deduce the most immunogenic subset of neoepitopes, and hence most promising of immunotherapy targets, T-cell assays are then run. Neoantigen discovery will therefore drive the design of neoantigen-specific vaccines and effective combination immunotherapies, and enable estimation of patient clinical response to treatments. Furthermore, neoantigen load estimation will therefore enable improved, personalized immunotherapies. Created using information from Schumacher and Schreiber (224), and Kvistborg et al. (225).

Importantly, as coding regions only account for $<2 \%$ of the human genome, understanding the impact of the even higher numbers of non-coding somatic mutations on cancer is a priority (228). A systematic analysis of 930 tumor whole genomes and transcriptomes revealed that a network of 193 non-coding loci were disrupted in $88 \%$ of tumors tested, indicating widespread effects of non-coding mutations across diverse cancers (228). Notably, in vitro studies showed that a non-coding mutation upstream of DAAM1 upregulated its expression, enhancing the invasiveness of tumor cells. Whether or not different tumor subtypes have common patterns of both non-coding and coding mutations, and the intricacy of the global transcriptional network linking non-coding mutations to tumor gene expression, requires further investigation (228).

Bioinformatic analysis of 10,000 tumors across 33 diverse cancers by the Cancer Research Institute (CRI) iAtlas suggested the existence of six major immune sub-types (229). Notably, C2 (IFN- $\gamma$ dominant) and C3 (inflammatory) subtypes were associated with good prognoses, while C4 (lymphocyte-depleted) and C6 (TGF- $\beta$ dominant) had the most negative prognoses. C4 and C6 both possess high M2 macrophage and low TIL level signatures, corroborating earlier studies where such immunosuppressed TMEs were associated with poor outcomes (229). Given that the TME provides important insight into patient prognosis and treatment response, tumor immune subtypes could provide an invaluable role for predicting disease outcome (229).

Patient-derived xenografts (PDXs) in which human tumor fragments are implanted into immunosuppressed mice show superior recapitulation of tumor heterogeneity, preservation of gene expression profiles and response to conventional chemotherapy than conventional xenografts (230). Thus, PDXs can facilitate personalized immunotherapy (230). Limitations include time (2-12 months are needed to establish the models) and metastases being difficult to observe (230). Consequently, humanized swine "oncopig" models are in development that better mirror the human condition, including pharmacokinetics (231).

A non-invasive and cost-effective radiomics-based approach was used recently to convert subjective CT imaging into objective data and thus enabling "biomarkers" predictive of patient response to immunotherapy (232). A radiomic score was established based upon TIL quantity estimated from 6 parameters. Application of this technique to 137 CTs of neck, bladder, lung, and head cancers revealed that higher scoring patients had $50 \%$ greater chance of survival in PD-1/PD-L1 trials than patients with lower scores (232). Such quantitation of personalized approaches to immunotherapy is promising and should be expanded.

FDA recently approved the first pan-tumor biomarker as a test for microsatellite instability (MSI) in connection with pembrolizumab (Keytruda) (233). This can test all advanced tumor types for highly elevated MSI-H and/or its underlying trigger, the DNA mismatch repair (dMMR) system, and thus enable exclusive molecular indication for immunotherapy (233). Approximately $4 \%$ of all tumors possess the hypermutational MSI-dMMR phenotype, significant levels of neoantigens/high immunogenicity and these are the cancers most susceptible to checkpoint blockades (233). Indeed, promising results have been reported for MMR-deficient pancreatic or prostate cancer 
patients: $30 \%$ showed complete response to therapy, $23 \%$ showed partial responses, and 17\% stabilized disease (233).

In conclusion, significant advances have been made in the personalization of immunotherapy. More work is required, however, to develop robust novel biomarkers (and their combination) to fulfill the potential of precision immunotherapy.

\section{CONCLUSIONS AND FUTURE PERSPECTIVES}

Whilst overwhelming evidence supports the potential of immunotherapies in clinical oncology, much cross-disciplinary work remains to be done to maximize patient benefit. First, there remains strong demand for realistic preclinical models that can predict optimal combinations to minimize adverse side-effects and toxicities, and better interrogate immunotherapy mechanisms of action. Emerging $3 \mathrm{D}$ in vitro models are sharpening our understanding of tumor-immune cell interactions. The effect upon T-lymphocyte functioning of tumor associated fibroblasts (TAFs), a significant component of the TME and enhancer of metastasis, was evaluated in breast cancer using chitosan-alginate scaffolds $(32,234)$. This study concluded that TAF inhibition may boost the efficacy of adoptive cellular therapies and that such 3D scaffold assays could bridge the gap between in vitro experiments and preclinical animal models for evaluation of immunotherapies $(234,235)$. Probing the intricate composition of the TME, therefore, is essential to broaden immunotherapeutic efficacy by validating novel targets and providing rationale for combination regimens. A complementary system is the tumor-containing Foxp3DTR mouse model in which Treg cells can be removed so immune cell inhibition can maximally be released prior to immunotherapy (236). Development of new models could benefit from physical and engineering approaches, for example, leading to: (i) unique, therapeutically exploitable physical features of TMEs (e.g., distinctive tumor cell glycocalyx, vasculature compression and stiffening of ECM) identified via PET, CT, or MRI imaging; (ii) improved drug delivery specificity and dosage via nanoparticles, bioscaffold cancer vaccines, RNAibased systems and implantable devices; and (iii) microfluidic "organs-on-chips" (237).

Second, of the possible some 20 different checkpoints exemplified by LAG-3, VISTA and TIM-3, only one (PD$1 / \mathrm{PD}-\mathrm{L} 1)$ is currently being fully exploited, so a lot remains to be evaluated. Even the functioning of PD-1 receptors needs to be understood better since whilst PD-1 blockade would be protective against solid tumors and non-T-cell cancers, it could exacerbate certain T-cell-derived tumors (36). Non-malignant cells (e.g., adipocytes, fibroblasts, pericytes and macrophages) within the TME that mediate intercellular communication between tumor cell subpopulations and can influence cancer progression could serve as viable immune-targets (2). Indeed, "tuning" and manipulation by IFN $\gamma$-facilitated conversion of macrophages of the M2/repairtype - responsible for repairing wounds-into M1/kill-type bolstered host anti-tumor response in mouse models, slowing, or stopping tumor growth (238). A meta-analysis of 90 immune checkpoint inhibitor trials found higher irAE incidence for anti-CTLA-4 $(\sim 54 \%)$, compared with anti-PD-1 $(\sim 27 \%)$ and anti-PD-L1 $(\sim 17 \%)$ (239). This would predict that checkpoint blockades targeting exclusively immune cells will have significantly higher irAE rates relative to those additionally targeting tumor cells (anti-PD-L1) (239). Future immunotherapy trial designs, therefore, should be adjusted accordingly.

Third, the issue of tumor resistance to immunotherapies needs in-depth understanding. Emerging evidence depicts tumor cells as "communities" or "rogue organs" with complex local population dynamics that produce both positive (commensalism and synergism) and negative (competition and parasitism) effects. To achieve long-term benefit, it is necessary to take into account both intra-tumoral heterogeneity and dynamics including treatment-induced changes (240). Patients with complete radiographical and pathological remission can still experience relapse due to minimal residual disease (MRD), especially with acute leukemia and aggressive lymphomas (241). In fact, instead of waiting for patients to relapse after effective therapy, MRD can be detected by flow cytometry then iteratively sampled and tested to establish its therapeutic susceptibility and optimal therapies could then be administered, even indefinitely (241). However, there is the inherent risk of cost and toxicity from overtreatment or incorrect treatment (241).

Fourth, gut microbiota has been implicated in regulating both immunotherapy responses and carcinogenesis (242). In murine models, Bifidobacterium was shown to induce anti-tumor immunity and enhance the therapeutic efficacy of PD-L1 blockade (243). Different mice displayed different microflora compositions, however, even when their genetic backgrounds were identical. Thus, identifying the most therapeutically favorable microbiota for different cancer types would seem a worthwhile effort (242). In another study, prevalent commensal communities of Bifidobacterium longum, Enterococcus faecium, and Collinsella aerofaciens were found in the $38 \%$ of metastatic melanoma patients responsive to PD-1 blockade, but were less abundant in the non-responsive patients (244). Koh et al. established a strong correlation between melanoma patient response to nivolumab and gut microbiota (245). Optimally responding patients had significant intestinal tract communities of Holdemania filiformis, Faecalibacterium prausnitzii, and Bacteroides thetaiotaomicron. Additionally, lung and kidney cancer patients treated with antibiotics saw nullified PD-1 blockade efficacy. The bacterium Akkermansia muciniphila was found in $60-70 \%$ of PD-1 blockade-treated patients with a PR or stable disease respectively, compared with only $34 \%$ in unresponsive patients (246). Thus, probiotic cocktails may ultimately be combined with immunotherapy in a personalized setting (245).

Fifth, further combination therapies are possible. For example, after surgical removal of a primary malignant tumor, there is still risk of recurrence including regrowth of micrometastases seeded from residual circulating tumor cells (CTCs) 
(247). Wang et al. conjugated platelets to an anti-PD-L1 checkpoint inhibitor and injected these intravenously into mouse 4T1 breast cancer model (247). Engineered platelets recruited the most immune cells to the surgery sites and thus delivered anti-PD-L1 with much greater accuracy and no significant irAE. Relative to controls, treated mice showed significantly enhanced survival. The technology is expected to find increasing applications, especially after optimizing issues like timing, dosage, and duration of combination regimens in relation to different tumor types $(32,248)$. In such optimization, for example, excessive elimination of immunosuppressive Treg cells, that would risk autoimmune reactions, should be avoided (249). Grinberg-Bleyer et al. recently showed that chemical ablation of c-Rel (a subunit of NF-kB transcription factor) via the muscle pain "dampener" pentoxifylline delayed melanoma development in murine models by repressing Treg-mediated immunosuppression (249). c-Rel inhibition boosted the efficacy of PD-1 blockade, allowing complete response rate in $\sim 20 \%$ of cases. Consequently, cRel inhibitors may become a standard component of future immune checkpoint blockade combinations (249). Another type of combination could involve natural products. For example, an extract from the Ayurvedic herb Andrographis paniculata was recently identified as a potent enhancer of NK cell activity in vitro and in vivo (250). High-throughput screening techniques promise to speed up identification of possible natural immunomodulators for combination immmunotherapy (251). Obesity-related factors, including insulin-like growth factor 1, adipokines and inflammation-mediators involved in systemic metabolic function, can directly interact with tumor cell metabolics (252). Consequently, obesity contributes to negative prognosis across many cancers by influencing tumor response to immunotherapy, and promoting tumor initiation and progression. Future combinations should, therefore, be increasingly guided by the personalized physiology of patients. Furthermore, introducing repurposed non-cancer drugs into immunotherapy could pay dividend. For example, diclofenac, an affordable, and readily accessible pain killer for rheumatoid arthritis, has yielded improved efficacies with chemotherapy and radiotherapy (253). The next leap in efficacy (possibly up to $\sim 80 \%$ patient survival rate) is anticipated to come from novel combination refinements, by embracing innovative biomarker-driven synergies and sequencing tumors using "The Cancer Genome Atlas." This promises to significantly improve our understanding of the molecular basis of tumor immunosuppressive mechanisms and could minimize irAEs $(254,255)$.

Sixth, rapidly advancing gene-editing technologies are also applicable to immunotherapy. PD-1 gene knockout by electroporation of sgRNA:Cas9 plasmids significantly repressed PD- 1 expression without compromising human T-cell viability in vitro. This technique efficiently disrupted $\mathrm{PD}-1$ and proved markedly more efficient in its use of electroporation, relative to current viral-based gene transfer approaches (256). The efficacy of ACT cellular therapies can also be improved by applying the same approach (256). Genetic engineering of NK cells for immunotherapy has historically been marred by low transduction efficiencies. Recently, however, mRNA electroporation successfully reprogrammed multiple NK modalities at high efficiency and reproducibility, without compromising cellular viability or phenotype (257). New efficacious avenues for adoptive NK-cell immunotherapy efficacy are thus opened, with potential to enhance many aspects of tumor targeting in vivo (257). Nanoparticles with gene therapeutics also offer hope. For example, mixing foxol mRNA nanocarriers with CAR-T cells to reprogram them, via "hit-andrun" transient expression, into more aggressive, higher efficacy and longer-lasting memory T-cells (258). Loss-of-function mutations alter the extent of malignant cells' vulnerability to T-lymphocyte-based immunotherapy (259). Patel et al. knockedout all known protein-encoding genes across the human genome by utilizing a genome-scale CRISPR-Cas9 library and profiling all genes where functional loss induced reduced CD8+ T-cell effector function (259). Identifying these genes could then serve as a "blueprint" to establish, at a genetic level, why certain patient tumor subsets are resistant to immunotherapies (259).

Seventh, advanced technology and artificial intelligence also promise to contribute significantly to the next wave of developments in immune-oncology. Existing supercomputers can facilitate (i) "big data" genomic analysis to uncover patient risk factors, (ii) optimizing dosages of combination drugs, (iii) modeling tissues, cells, and drug interactions to identify novel medicines, and (iv) discovery of therapeutically exploitable relationships within complex cellular networks (260). Quantum computing, with superior processing power and speed, also promises to bring unprecedented sub-atomic detail to our understanding of immunotherapeutic effects (261).

Finally, we should stress that significant safety concerns remain in both mono- and combination immunotherapies, and much more work is required to limit and control the undesirable side effects $(34,35,262-264)$. As well as pre-determining the subpopulations of patients likely to benefit from given treatment regimens, therefore, personalized medication should incorporate predictive biomarkers for identifying possible autoimmune response risk (262).

\section{AUTHOR CONTRIBUTIONS}

HM performed the literature search supported by MD. HM wrote the main text and prepared the illustrations. MD and HM finalized the manuscript.

\section{SUPPLEMENTARY MATERIAL}

The Supplementary Material for this article can be found online at: https://www.frontiersin.org/articles/10.3389/fonc. 2018.00315/full\#supplementary-material 


\section{REFERENCES}

1. Hanahan D, Weinberg RA. Hallmarks of cancer: the next generation. Cell (2011) 144:646-74. doi: 10.1016/j.cell.2011.02.013

2. Balkwill FR, Capasso M, Hagemann T. The tumor microenvironment at a glance. J Cell Sci. (2012) 125:5591-6. doi: 10.1242/jcs. 116392

3. Tang H, Qiao J, Fu Y-X. Immunotherapy and tumor microenvironment. Cancer Lett. (2016) 370:85-90. doi: 10.1016/j.canlet.2015. 10.009

4. Karpanen T, Olweus J. The potential of donor T-cell repertoires in neoantigen-targeted cancer immunotherapy. Front Immunol. (2017) 8:1718. doi: 10.3389/fimmu.2017.01718

5. Jeanbart L, Swartz MA. Engineering opportunities in cancer immunotherapy. Proc Natl Acad Sci USA (2015) 112:14467-72. doi: 10.1073/pnas.1508516112

6. Hasmim M, Messai Y, Ziani L, Thiery J, Bouhris J-H, Noman MZ, et al. Critical role of tumor microenvironment in shaping NK cell functions: implication of hypoxic stress. Front Immunol. (2015) 6:482. doi: 10.3389/fimmu.2015.00482

7. Ferris RL. Hiding in Plain Sight: Mechanisms of Tumor Immune Evasion. Medscape (2016) Available online at: http://www.medscape.org/viewarticle/ 841945_2 (Accessed August 14, 2016).

8. Derbal Y. Perspective on the dynamics of cancer. Theor Biol Med Model. (2017) 14:18. doi: 10.1186/s12976-017-0066-5

9. Baginska J, Viry E, Paggetti J, Medves S, Berchem G, Moussay E, et al. The critical role of the tumor microenvironment in shaping natural killer cell-mediated anti-tumor immunity. Front Immunol (2013) 4:490. doi: 10.3389/fimmu.2013.00490

10. Hargadon KM. Tumor-altered dendritic cell function: implications for anti-tumor immunity. Front Immunol. (2013) 4:192. doi: 10.3389/fimmu.2013.00192

11. Chikuma S. CTLA-4, an essential immune-checkpoint for T-cell activation. Curr Top Microbiol Immunol. (2017) 410:99-126. doi: 10.1007/82_2017_61

12. Baumeister SH, Freeman GJ, Dranoff G, Sharpe AH. Coinhibitory pathways in immunotherapy for cancer. Annu Rev Immunol. (2016) 34:539-573. doi: 10.1146/annurev-immunol-032414-112049

13. Mahoney KM, Rennert PD, Freeman GJ. Combination cancer immunotherapy and new immunomodulatory targets. Nat Rev Drug Discov. (2015) 14:561-84. doi: 10.1038/nrd4591

14. Melero I, Berman DM, Aznar MA, Korman AJ, Pérez Gracia JL, Haanen J. Evolving synergistic combinations of targeted immunotherapies to combat cancer. Nat Rev Cancer (2015) 15:457-72. doi: 10.1038/nrc3973

15. Khalil DN, Smith EL, Brentjens RJ, Wolchok JD. The future of cancer treatment: immunomodulation, CARs and combination immunotherapy. Nat Rev Clin Oncol. (2016) 13:273-90. doi: 10.1038/nrclinonc.2016.25

16. Farkona S, Diamandis EP, Blasutig IM. Cancer immunotherapy: the beginning of the end of cancer? BMC Med. (2016) 14:73. doi: 10.1186/s12916-016-0623-5

17. Mellman I, Coukos G, Dranoff G. Cancer immunotherapy comes of age. Nature (2011) 480:480-9. doi: 10.1038/nature10673

18. Vasaturo A, Di Blasio S, Peeters DGA, de Koning CCH, de Vries JM, Figdor $\mathrm{CG}$, et al. Clinical implications of co-inhibitory molecule expression in the tumor microenvironment for DC vaccination: a game of stop and go. Front Immunol. (2013) 4:417. doi: 10.3389/fimmu.2013.00417

19. Ansell SM, Lesokhin AM, Borrello I, Halwani A, Scott EC, Gutierrez $\mathrm{M}$, et al. PD-1 Blockade with nivolumab in relapsed or refractory Hodgkin's lymphoma. N Engl J Med. (2014) 372:141206100011003. doi: 10.1056/NEJMoa1411087

20. Adams JL, Smothers J, Srinivasan R, Hoos A. Big opportunities for small molecules in immuno-oncology. Nat Rev Drug Discov. (2015) 14:603-22. doi: $10.1038 / \mathrm{nrd} 4596$

21. Guo ZS, Bartlett DL. Oncolytic viruses as platform for multimodal cancer therapeutics: a promising land. Cancer Gene Ther. (2014) 21:261-3. doi: $10.1038 /$ cgt.2014.31

22. Tchekmedyian N, Gray JE, Creelan BC, Chiappori AA, Beg AA, Soliman $\mathrm{H}$, et al. Propelling immunotherapy combinations into the clinic. Oncology (2015) 29: 990-1002.
23. Garon EB, Rizvi NA, Hui R, Leighl N, Balmanoukian AS, Eder JP, et al. Pembrolizumab for the treatment of non-small-cell lung cancer. $N$ Engl J Med. (2015) 372:2018-28. doi: 10.1056/NEJMoa1501824

24. Smyth MJ, Ngiow SF, Ribas A, Teng MWL. Combination cancer immunotherapies tailored to the tumour microenvironment. Nat Rev Clin Oncol. (2016) 13:143-58. doi: 10.1038/nrclinonc.2015.209

25. Cogdill AP, Andrews MC, Wargo JA. Hallmarks of response to immune checkpoint blockade. Br J Cancer (2017) 117:1-7. doi: 10.1038/bjc.2017.136

26. Yaddanapudi K, Mitchell RA, Eaton JW. Cancer vaccines: looking to the future. Oncoimmunology (2013) 2:e23403. doi: 10.4161/onci.23403

27. Couzin-Frankel J. Autoimmune diseases surface after cancer treatment. Science (2017) 358:852. doi: 10.1126/science.358.6365.852

28. Isakov N. Immune checkpoint-targeted therapy: cancer and autoimmune diseases represent two sides of the same coin. J Autoimmune Disord. (2016) 2:2. doi: 10.21767/2471-8513.100017

29. Joshi M., Whitelaw BC, Palomar MTP, Wu Y, Carroll PV. Immune checkpoint inhibitor-related hypophysitis and endocrine dysfunction: clinical review. Clin Endocrinol. (2016) 85:331-9. doi: 10.1111/cen.13063

30. O’Day SJ, Maio M, Chiarion-Sileni V, Gajewski TF, Pehamberger H, Bondarenko IN, et al. Efficacy and safety of ipilimumab monotherapy in patients with pretreated advanced melanoma: a multicenter single-arm phase II study. Ann Oncol Off J Eur Soc Med Oncol. (2010) 21:1712-7. doi: 10.1093/annonc/mdq013

31. Postow MA, Chesney J, Pavlick AC, Robert C, Grossmann K, McDermott D, et al. Nivolumab and ipilimumab versus ipilimumab in untreated melanoma. N Engl J Med. (2015) 372:2006-17. doi: 10.1056/NEJMoa1414428

32. Alsaab HO, Sau S, Alzhrani R, Tatiparti K, Bhise K, Kashaw SK, et al. PD1 and PD-L1 checkpoint signaling inhibition for cancer immunotherapy: mechanism, combinations, and clinical outcome. Front Pharmacol. (2017) 8:561. doi: 10.3389/fphar.2017.00561

33. Richtel M. Immune System, Unleashed by Cancer Therapies, Can Attack Organs. New York Times (2016) Available online at: https://www.nytimes. com/2016/12/03/health/immunotherapy-cancer.html (Accessed December 3, 2016).

34. Champiat S, Dercle L, Ammari S, Massard C, Hollebecque A, Postel-Vinay $\mathrm{S}$, et al. Hyperprogressive disease is a new pattern of progression in cancer patients treated by anti-PD-1/PD-L1. Clin Cancer Res. (2017) 23:1920-8. doi: 10.1158/1078-0432.CCR-16-1741

35. Kato S, Goodman A, Walavalkar V, Barkauskas DA, Sharabi A, Kurzrock R. Hyperprogressors after immunotherapy: analysis of genomic alterations associated with accelerated growth rate. Clin Cancer Res. (2017) 23:4242-50. doi: 10.1158/1078-0432.CCR-16-3133

36. Ludin A, Zon LI. Cancer immunotherapy: the dark side of PD-1 receptor inhibition. Nature (2017) 552:41-2. doi: 10.1038/nature24759

37. Hodi FS, Lee S, McDermott DF, Rao UN, Butterfield LH, Tarhini AA, et al. Ipilimumab plus sargramostim vs ipilimumab alone for treatment of metastatic melanoma. JAMA (2014) 312:1744. doi: 10.1001/jama.2014.13943

38. Combination immunotherapy. In: Biomarkers \& Immuno-Oncology World Congress 2017. Combination Immunotherapy. Philadelphia, PA. Available online at: http://www.giiconference.com/chi374399/combinationimmunotherapy.shtml (Accessed April 6, 2017).

39. Combination immunotherapy: an emerging paradigm in cancer therapeutics. Cancer Netw. (2015) 29:1005-6.

40. Hoos A. Development of immuno-oncology drugs - from CTLA4 to PD1 to the next generations. Nat Rev Drug Discov. (2016) 15:235-47. doi: $10.1038 / \mathrm{nrd} .2015 .35$

41. Dempke WCM, Fenchel K, Uciechowski P, Dale SP. Second- and thirdgeneration drugs for immuno-oncology treatment-The more the better? Eur J Cancer (2017) 74:55-72. doi: 10.1016/j.ejca.2017.01.001

42. OncoQuest Inc. Overview. OncoQuest Inc (2016) Available online at: http:// oncoquestinc.com/Products-Under-Development/Overview (Accessed August 15, 2016).

43. Johnson DB, Peng C, Sosman JA. Nivolumab in melanoma: latest evidence and clinical potential. Ther Adv Med Oncol. (2015) 7:97-106. doi: $10.1177 / 1758834014567469$

44. Larkin J, Chiarion-Sileni V, Gonzalez R, Grob JJ, Cowey CL, Lao CD, et al. Combined nivolumab and ipilimumab or monotherapy in untreated melanoma. N Engl J Med. (2015) 373:23-34. doi: 10.1056/NEJMoa1504030 
45. Antonia S, Goldberg SB, Balmanoukian A, Chaft JE, Sanborn RE, Gupta A, et al. Safety and antitumour activity of durvalumab plus tremelimumab in non-small cell lung cancer: a multicentre, phase 1b study. Lancet Oncol. (2016) 17:299-308. doi: 10.1016/S1470-2045(15)00544-6

46. Santa-Maria C. MEDI4736 and Tremelimumab in Treating Patients With Metastatic HER2 Negative Breast Cancer. (2017) Available online at: https:// clinicaltrials.gov/ct2/show/NCT02536794 (Accessed October 28, 2017).

47. Long GV, Atkinson V, Cebon JS, Jameson MB, Fitzharris BM, McNeil $\mathrm{CM}$, et al. Standard-dose pembrolizumab in combination with reduceddose ipilimumab for patients with advanced melanoma (KEYNOTE029): an open-label, phase 1b trial. Lancet Oncol. (2017) 18:1202-10. doi: 10.1016/S1470-2045(17)30428-X

48. Choueiri TK, Hodi FS, Thompson JA, McDermott DF, Hwu WJ, Lawrence DP, et al. Pembrolizumab (pembro) plus low-dose ipilimumab (ipi) for patients (pts) with advanced renal cell carcinoma (RCC): Phase 1 KEYNOTE-029 study. J Clin Oncol. (2017) 35:510. doi: 10.1200/JCO.2017.35.6_suppl.510

49. Santabarbara G, Maione P, Rossi A, Palazzolo G, Gridelli C. The role of pembrolizumab in the treatment of advanced non-small cell lung cancer. Ann Transl Med. (2016) 4:215. doi: 10.21037/atm.2016.05.64

50. An Investigational Immuno-therapy Study to Assess the Safety, Tolerability and Effectiveness of Anti-LAG-3 With and Without Anti-PD-1 in the Treatment of Solid Tumors. Bristol-Myers Squibb (2017) Available online at: https://clinicaltrials.gov/ct2/show/NCT01968109 (Accessed October 28, 2017).

51. Bajor DL, Mick R, Riese MJ, Richman LP, Xu X, Torigian DA, et al. Abstract CT137: Combination of agonistic CD40 monoclonal antibody CP-870,893 and anti-CTLA-4 antibody tremelimumab in patients with metastatic melanoma. Cancer Res. (2015) 75:CT137. doi: 10.1158/1538-7445.AM2015-CT137

52. Ansell S. Nivolumab With or Without Varlilumab in Treating Patients With Relapsed or Refractory Aggressive B-cell Lymphomas. National Cancer Institute (2017) Available online at: https://clinicaltrials.gov/ct2/show/ NCT03038672 (Accessed October 28, 2017).

53. A Phase 1 Study to Evaluate MEDI6383 Alone and in Combination With MEDI4736 in Adult Subjects With Select Advanced Solid Tumors. MedImmune LLC (2017) Available online at: https://clinicaltrials.gov/ct2/ show/NCT02221960 (Accessed October 28, 2017).

54. Study of MK-4166 and MK-4166 in Combination With Pembrolizumab (MK3475) in Participants With Advanced Solid Tumors (MK-4166-001). Merck Sharp \& Dohme Corp (2017) Available online at: https://clinicaltrials.gov/ show/NCT02132754 (Accessed October 28, 2017).

55. Tolcher AW, Sznol M, Hu-Lieskovan S, Papadopoulos KP, Patnaik A, Rasco DW, et al. Phase Ib study of utomilumab (PF-05082566), a 4$1 \mathrm{BB} / \mathrm{CD} 137$ agonist, in combination with pembrolizumab (MK-3475) in patients with advanced solid tumors. Clin Cancer Res. (2017) 23:5349-57. doi: 10.1158/1078-0432.CCR-17-1243

56. Advani A. Blinatumomab and Combination Chemotherapy or Dasatinib, Prednisone, and Blinatumomab in Treating Older Patients With Acute Lymphoblastic Leukemia. (2017) Available online at: https://clinicaltrials.gov/ ct2/show/NCT02143414 (Accessed October 15, 2017).

57. Lynne Johnson M. Syndax Announces Results from Phase 2 ENCORE 601 Trial of Entinostat in Combination with KEYTRUDA®) (pembrolizumab) for the Treatment of Advanced Melanoma (NASDAQ:SNDX). Syndax (2017) Available online at: http://ir.syndax.com/releasedetail.cfm?releaseid= 1026895 (Accessed October 14, 2017).

58. Brahmer J, Nieva J. NCT01928576 Clinical Trial - National Cancer Institute. National Cancer Institute (2017) Available online at: https://www.cancer. gov/about-cancer/treatment/clinical-trials/search/view?cdrid $=751963$ (Accessed October 14, 2017).

59. Atkins MB, Plimack ER, Puzanov I, Fishman MN, McDermott D, Cho DC, et al. Axitinib in combination with pembrolizumab in patients (pts) with advanced renal cell carcinoma (aRCC): Preliminary safety and efficacy results. Ann Oncol. (2016) 27. doi: 10.1093/annonc/mdw373.01

60. Salama AKS, Moschos SJ. Next steps in immuno-oncology: enhancing antitumor effects through appropriate patient selection and rationally designed combination strategies. Ann Oncol. (2016) 28:mdw534. doi: 10.1093/annonc/mdw534
61. Chowdhury S. A Phase I/II Study to Assess the Safety and Efficacy of Pazopanib (PAZ) and Pembrolizumab (PEM) in Patients (pts) with Advanced Renal Cell Carcinoma (aRCC). ASCO Annual Meeting (2017) Available online at: http://meetinglibrary.asco.org/record/152938/abstract (Accessed October 15, 2017).

62. Goel S, DeCristo MJ, Watt AC, BrinJones H, Sceneay J, Li BB, et al. CDK4/6 inhibition triggers anti-tumour immunity. Nature (2017) 548:4715. doi: 10.1038/nature23465

63. Robert C, Schachter J, Long GV, Arance A, Grob JJ, Mortier L, et al. Pembrolizumab versus Ipilimumab in advanced melanoma. $N$ Engl J Med. (2015) 372:2521-32. doi: 10.1056/NEJMoa1503093

64. Gangadhar TC, Hamid O, Smith DC, Bauer TM, Wasser JS, Luke JJ, et al. Preliminary results from a Phase I/II study of epacadostat (incb024360) in combination with pembrolizumab in patients with selected advanced cancers. J Immunother Cancer (2015) 3:07. doi: 10.1186/2051-1426-3-S2-O7

65. John LB, Devaud C, Duong CPM, Yong CS, Beavis PA, Haynes NM, et al. Anti-PD-1 antibody therapy potently enhances the eradication of established tumors by gene-modified T cells. Clin Cancer Res. (2013) 19:5636-46. doi: 10.1158/1078-0432.CCR-13-0458

66. Haanen JBAG, Harview CL, Yearley JH, Shintaku IP, Taylor EJM, Robert L, et al. Converting cold into hot tumors by combining immunotherapies. Cell (2017) 170:1055-6. doi: 10.1016/j.cell.2017.08.031

67. Antonios JP, Soto H, Everson RG, Orpilla J, Moughon D, Shin N, et al. PD-1 blockade enhances the vaccination- induced immune response in glioma. JCI Insight (2016) 1:e87059. doi: 10.1172/jci.insight.87059

68. Morgensztern D. A Study of Combination Therapies With Viagenpumatucel-L (HS-110) in Patients With Non-Small Cell Lung Cancer. (2017) Available online at: https://clinicaltrials.gov/ct2/show/NCT02439450 (Accessed October 15, 2017).

69. Curti B, Richards J, Hallmeyer S, Andtbacka R. The MITCI (Phase 1b) study: A novel immunotherapy combination of intralesional Coxsackievirus A21 and systemic ipilimumab in advanced melanoma patients with or without previous immune checkpoint therapy treatment. In: $A A C R$ Annual Meeting 2017 Washington, DC (2017). Available online at: http:// www.abstractsonline.com/pp8/\#!/4292/presentation/12329 (Accessed April 7, 2017).

70. A Study of Atezolizumab in Combination With Carboplatin Plus (+) Paclitaxel With or Without Bevacizumab Compared With Carboplatin+Paclitaxel+Bevacizumab in Participants With Stage IV Non-Squamous Non-Small Cell Lung Cancer (NSCLC). (2017) Available online at: https://clinicaltrials.gov/ct2/show/study/NCT02366143 (Accessed October 15, 2017).

71. Gandhi L, Rodríguez-Abreu D, Gadgeel S, Esteban E, Felip E, De Angelis F, et al. Pembrolizumab plus chemotherapy in metastatic non-small-cell lung cancer. N Engl J Med. (2018) 378:2078-92. doi: 10.1056/NEJMoa1801005

72. Kok M, Horlings HM, Van de Vijver K. Adaptive phase II randomized noncomparative trial of nivolumab after induction treatment in triple negative breast cancer: TONIC-trial OncologyPRO. Oncol Pro. (2017) 8(Suppl. 5): v605-49. doi: 10.1093/annonc/mdx440

73. Chao J, Chen Y-J, Frankel PH, Chung VM, Lim D, Li D, et al. Combining pembrolizumab and palliative radiotherapy in gastroesophageal cancer to enhance anti-tumor T-cell response and augment the abscopal effect. J Clin Oncol. (2017) 35:TPS220-TPS220. doi: 10.1200/JCO.2017.35.4_suppl.TPS220

74. He C, Duan X, Guo N, Chan C, Poon C, Weichselbaum RR, et al. Core-shell nanoscale coordination polymers combine chemotherapy and photodynamic therapy to potentiate checkpoint blockade cancer immunotherapy. Nat Commun. (2016) 7:12499. doi: 10.1038/ncomms 12499

75. Duan X, Chan C, Guo N, Han W, Weichselbaum RR, Lin W. Photodynamic therapy mediated by nontoxic core-shell nanoparticles synergizes with immune checkpoint blockade to elicit antitumor immunity and antimetastatic effect on breast cancer. J Am Chem Soc. (2016) 138:1668695. doi: 10.1021/jacs.6b09538

76. Liu Y, Maccarini P, Palmer GM, Etienne W, Zhao Y, Lee C-T, et al. Synergistic immuno photothermal nanotherapy (SYMPHONY) for the treatment of unresectable and metastatic cancers. Sci Rep. (2017) 7:8606. doi: 10.1038/s41598-017-09116-1 
77. Morrissey K, Yuraszeck T, Li C-C, Zhang Y, Kasichayanula S. Immunotherapy and novel combinations in oncology: current landscape, challenges, and opportunities. Clin Transl Sci. (2016) 9:89-104. doi: $10.1111 /$ cts. 12391

78. Bates SE. Refining immunotherapy approvals. Clin Cancer Res. (2017) 23:4948-9. doi: 10.1158/1078-0432.CCR-17-2025

79. Hughes PE, Caenepeel S, Wu LC. Targeted therapy and checkpoint immunotherapy combinations for the treatment of cancer. Trends Immunol. (2016) 37:462-76. doi: 10.1016/j.it.2016.04.010

80. Seba R. Soluble Immune Checkpoint Markers: Understanding Therapeutic Antibody Response in Immuno-oncology. Gen (2017) Available online at: http://www.genengnews.com/gen-articles/soluble-immunecheckpointmarkers/5983?utm_medium $=$ newsletter\&utm_source=GEN+ Daily + News + Highlights\&utm_content $=01 \& u t m_{-}$campaign $=G E N+$ Daily + News+Highlights_20170703 (Accessed July 8, 2017).

81. Topalian SL, Wolchok JD, Chan TA, Mellman I, Palucka K, Banchereau J, et al. Immunotherapy: the path to win the war on cancer? Cell (2015) 161:185-6. doi: 10.1016/j.cell.2015.03.045

82. Anderson AC. Tim-3: an emerging target in the cancer immunotherapy landscape. Cancer Immunol Res. (2014) 2:393-8. doi: 10.1158/2326-6066.CIR-14-0039

83. Durham NM, Nirschl CJ, Jackson CM, Elias J, Kochel CM, Anders RA, et al. Lymphocyte activation gene 3 (LAG-3) modulates the ability of CD4 T-cells to be suppressed in vivo. PLoS ONE (2014) 9:e109080. doi: 10.1371/journal.pone.0109080

84. Wang L, Le Mercier I, Putra J, Chen W, Liu J, Schenk AD, et al. Disruption of the immune-checkpoint VISTA gene imparts a proinflammatory phenotype with predisposition to the development of autoimmunity. Proc Natl Acad Sci USA. (2014) 111:14846-51. doi: 10.1073/pnas.1407447111

85. Anderson AC, Joller N, Kuchroo VK. Lag-3, Tim-3, and TIGIT: co-inhibitory receptors with specialized functions in immune regulation. Immunity (2016) 44:989-1004. doi: 10.1016/j.immuni.2016.05.001

86. Raper V. To Root out cancer immunotherapies dig deeper. Gen (2018) 38:26-28.

87. Tinoco R, Carrette F, Barraza ML, Otero DC, Magaña J, Bosenberg MW, et al. PSGL-1 is an immune checkpoint regulator that promotes $\mathrm{T}$ cell exhaustion. Immunity (2016) 44:1190-203. doi: 10.1016/j.immuni.2016.04.015

88. Gao J, Ward JF, Pettaway CA, Shi LZ, Subudhi SK, Vence LM, et al. VISTA is an inhibitory immune checkpoint that is increased after ipilimumab therapy in patients with prostate cancer. Nat Med. (2017) 23:551-5. doi: $10.1038 / \mathrm{nm} .4308$

89. Suntharalingam G, Perry MR, Ward S, Brett SJ, Castello-Cortes A, Brunner MD, et al. Cytokine storm in a phase 1 trial of the antiCD28 monoclonal antibody TGN1412. N Engl J Med. (2006) 355:1018-28. doi: 10.1056/NEJMoa063842

90. Bartkowiak T, Curran MA. 4-1BB agonists: multi-potent potentiators of tumor immunity. Front Oncol. (2015) 5:117. doi: 10.3389/fonc.2015.00117

91. Shindo Y, Yoshimura K, Kuramasu A, Watanabe Y, Ito H, Kondo T, et al. Combination immunotherapy with 4-1BB activation and PD-1 blockade enhances antitumor efficacy in a mouse model of subcutaneous tumor. Anticancer Res. (2015) 35:129-36.

92. McKee SJ, Doff BL, Soon MSF, Mattarollo SR. Therapeutic efficacy of $4-1 \mathrm{BB}$ costimulation is abrogated by PD-1 blockade in a model of spontaneous B-cell lymphoma. Cancer Immunol Res. (2017) 5:191-7. doi: 10.1158/2326-6066.CIR-16-0249

93. Messenheimer DJ, Jensen SM, Afentoulis ME, Wegmann KW, Feng Z, Friedman DJ, et al. Timing of PD-1 blockade is critical to effective combination immunotherapy with anti-OX40. Clin Cancer Res. (2017) 23:6165-77. doi: 10.1158/1078-0432.CCR-16-2677

94. Postow MA, Callahan MK, Barker CA, Yamada Y, Yuan J, Kitano S, et al. Immunologic correlates of the abscopal effect in a patient with melanoma. N Engl J Med. (2012) 366:925-31. doi: 10.1056/NEJMoa11 12824

95. Sharabi AB, Tran PT, Lim M, Drake CG, Deweese TL. Stereotactic radiation therapy combined with immunotherapy: augmenting the role of radiation in local and systemic treatment. Oncology (2015) 29:331-40.
96. Levitan D. Combined immunotherapy, thoracic RT carries moderate toxicity in lung cancer. Cancer Netw. (2017). Available online at: http:// www.cancernetwork.com/radiation-oncology/combined-immunotherapythoracic-rt-carries-moderate-toxicity-lung-cancer

97. Hiniker SM, Maecker HT, Knox SJ. Predictors of clinical response to immunotherapy with or without radiotherapy. J Radiat Oncol. (2015) 4:33945. doi: 10.1007/s13566-015-0219-2

98. Bracci L, Schiavoni G, Sistigu A, Belardelli F. Immune-based mechanisms of cytotoxic chemotherapy: implications for the design of novel and rationalebased combined treatments against cancer. Cell Death Differ. (2014) 21:1525. doi: $10.1038 /$ cdd.2013.67

99. Reck M, Bondarenko I, Luft A, Serwatowski P, Barlesi F, Chacko R, et al. Ipilimumab in combination with paclitaxel and carboplatin as firstline therapy in extensive-disease-small-cell lung cancer: results from a randomized, double-blind, multicenter phase 2 trial. Ann Oncol. (2013) 24:75-83. doi: 10.1093/annonc/mds213

100. Wang W, Wu L, Zhang J, Wu H, Han E, Guo Q. Chemoimmunotherapy by combining oxaliplatin with immune checkpoint blockades reduced tumor burden in colorectal cancer animal model. Biochem Biophys Res Commun. (2017) 487:1-7. doi: 10.1016/j.bbrc.2016.12.180

101. Kuznar W. Priming immune system before nivolumab improves response in metastatic TNBC. Target Oncol. (2017) Available online at: https://www. targetedonc.com/conference/esmo-2017/priming-immune-system-beforenivolumab-improves-response-in-metastatic-tnbc (Accessed September 10, 2017)

102. Benson Z, Manjili SH, Habibi M, Guruli G, Toor AA, Payne KK, et al. Conditioning neoadjuvant therapies for improved immunotherapy of cancer. Biochem Pharmacol. (2017) 145:12-7. doi: 10.1016/j.bcp.2017.08.007

103. Huehls AM, Coupet TA, Sentman CL. Bispecific T-cell engagers for cancer immunotherapy. Immunol Cell Biol. (2015) 93:290-6. doi: $10.1038 /$ icb. 2014.93

104. Neves H, Kwok HF. Recent advances in the field of anti-cancer immunotherapy. BBA Clin. (2015) 3:280-8. doi: 10.1016/j.bbacli.2015.04.001

105. Le Jeune $C$, Thomas X. Potential for bispecific T-cell engagers: role of blinatumomab in acute lymphoblastic leukemia. Drug Des Devel Ther. (2016) 10:757-65. doi: 10.2147/DDDT.S83848

106. Topp MS, Gökbuget N, Stein AS, Zugmaier G, O'Brien S, Bargou RC, et al. Safety and activity of blinatumomab for adult patients with relapsed or refractory B-precursor acute lymphoblastic leukaemia: a multicentre, single-arm, phase 2 study. Lancet Oncol. (2015) 16:57-66. doi: 10.1016/S1470-2045(14)71170-2

107. Gökbuget N, Kelsh M, Chia V, Advani A, Bassan R, Dombret $\mathrm{H}$, et al. Blinatumomab vs historical standard therapy of adult relapsed/refractory acute lymphoblastic leukemia. Blood Cancer J. (2016) 6:e473. doi: 10.1038/bcj.2016.84

108. Wu J, Fu J, Zhang M, Liu D. Blinatumomab: a bispecific $\mathrm{T}$ cell engager (BiTE) antibody against CD19/CD3 for refractory acute lymphoid leukemia. J Hematol Oncol. (2015) 8:104. doi: 10.1186/s13045-015-0195-4

109. Kwiatkowska-Borowczyk EP, Gabka-Buszek A, Jankowski J, Mackiewicz A. Immunotargeting of cancer stem cells. Contemp Oncol. (2015) 19:A52-9. doi: 10.5114/wo.2014.47129

110. Ecker DM, Jones SD, Levine HL, Li J, Ecker D, Jones $\mathrm{S}$, et al. The therapeutic monoclonal antibody market. MAbs (2015) 7:9-14. doi: $10.4161 / 19420862.2015 .989042$

111. Strauss J, Heery CR, Schlom J, Madan RA, Cao L, Kang Z, et al. Phase I trial of M7824 (MSB0011359C), a bifunctional fusion protein targeting PD-L1 and TGF $\beta$, in advanced solid tumors. Clin Cancer Res. (2018) 24:1287-95. doi: 10.1158/1078-0432.CCR-17-2653

112. Park J, Thomas S, Munster PN. Epigenetic modulation with histone deacetylase inhibitors in combination with immunotherapy. Epigenomics (2015) 7:641-52. doi: 10.2217/epi.15.16

113. Nervi C, De Marinis E, Codacci-Pisanelli G. Epigenetic treatment of solid tumours: a review of clinical trials. Clin Epigenetics (2015) 7:127. doi: 10.1186/s13148-015-0157-2

114. Weintraub K. Take two: Combining immunotherapy with epigenetic drugs to tackle cancer. Nat Med. (2016) 22:8-10. doi: 10.1038/nm0116-8 
115. Mazzone R, Zwergel C, Mai A, Valente S. Epi-drugs in combination with immunotherapy: a new avenue to improve anticancer efficacy. Clin Epigenetics (2017) 9:59. doi: 10.1186/s13148-017-0358-y

116. Ørskov AD, Treppendahl MB, Skovbo A, Holm MS, Friis LS, Hokland M, et al. Hypomethylation and up-regulation of PD-1 in T cells by azacytidine in MDS/AML patients: a rationale for combined targeting of PD-1 and DNA methylation. Oncotarget (2015) 6:9612-26. doi: 10.18632/oncotarget.3324

117. Balachandran VP, Cavnar MJ, Zeng S, Bamboat ZM, Ocuin LM, Obaid H, et al. Imatinib potentiates antitumor $\mathrm{T}$ cell responses in gastrointestinal stromal tumor through the inhibition of Ido. Nat Med. (2011) 17:1094-100. doi: $10.1038 / \mathrm{nm} .2438$

118. Sapkota B, Hill CE, Pollack BP. Vemurafenib enhances MHC induction in BRAF(V600E) homozygous melanoma cells. Oncoimmunology (2013) 2:e22890. doi: 10.4161/onci.22890

119. Ribas A, Hodi FS, Callahan M, Konto C, Wolchok J. Hepatotoxicity with combination of vemurafenib and ipilimumab. N Engl J Med. (2013) 368:1365-6. doi: 10.1056/NEJMc1302338

120. Hu-Lieskovan S, Robert L, Homet Moreno B, Ribas A. Combining targeted therapy with immunotherapy in BRAF-mutant melanoma: promise and challenges. J Clin Oncol. (2014) 32:2248-54. doi: 10.1200/JCO.2013.52.1377

121. Kaneda MM, Messer KS, Ralainirina N, Li H, Leem CJ, Gorjestani S, et al. $\mathrm{PI} 3 \mathrm{~K} \gamma$ is a molecular switch that controls immune suppression. Nature (2016) 539:437-42. doi: 10.1038/nature19834

122. De Henau O, Rausch M, Winkler D, Campesato LF, Liu C, Cymerman DH, et al. Overcoming resistance to checkpoint blockade therapy by targeting PI3K $\gamma$ in myeloid cells. Nature (2016) 539:443-7. doi: 10.1038/nature20554

123. Neubert NJ, Schmittnaegel M, Bordry N, Nassiri S, Wald N, Martignier C, et al. T cell-induced CSF1 promotes melanoma resistance to PD1 blockade. Sci Transl Med. (2018) 10:eaan3311. doi: 10.1126/scitranslmed.aan3311

124. Mok S, Koya RC, Tsui C, Xu J, Robert L, Wu L, et al. Inhibition of CSF-1 receptor improves the antitumor efficacy of adoptive cell transfer immunotherapy. Cancer Res. (2014) 74:153-61. doi: 10.1158/0008-5472.CAN-13-1816

125. Heuser C, Gotot J, Piotrowski EC, Philipp M-S, Courrèges CJF, Otte MS, et al. Prolonged IKK $\beta$ inhibition improves ongoing CTL antitumor responses by incapacitating regulatory T cells. Cell Rep. (2017) 21:578-86. doi: 10.1016/j.celrep.2017.09.082

126. Patnaik A, Rosen LS, Tolaney SM, Tolcher AW, Goldman JW, Gandhi L, et al. Efficacy and safety of abemaciclib, an inhibitor of CDK4 and CDK6, for patients with breast cancer, non-small cell lung cancer, and other solid tumors. Cancer Discov. (2016) 6:740-53. doi: 10.1158/2159-8290.CD-16-0095

127. Mouraviev V, Mariados N, Albala D, Concepcion RS, Shore ND, Sims $\mathrm{RB}$, et al. The rationale for optimal combination therapy with sipuleucel$\mathrm{T}$ for patients with castration-resistant prostate cancer. Rev Urol. (2014) 16:122-30. doi: 10.3909/riu0637

128. Ali OA, Lewin SA, Dranoff G, Mooney DJ. Vaccines combined with immune checkpoint antibodies promote cytotoxic T-cell activity and tumor eradication. Cancer Immunol Res. (2016) 4:95-100. doi: 10.1158/2326-6066.CIR-14-0126

129. Soares KC, Rucki AA, Wu AA, Olino K, Xiao Q, Chai Y, et al. PD1/PD-L1 blockade together with vaccine therapy facilitates effector $\mathrm{T}$ cell infiltration into pancreatic tumors. J Immunother. (2015) 38:1-11. doi: 10.1097/CJI.0000000000000062

130. Perica K, Varela JC, Oelke M, Schneck J. Adoptive T cell immunotherapy for cancer. Rambam Maimonides Med J. (2015) 6:e0004. doi: 10.5041/RMMJ.10179

131. Rosenberg SA, Restifo NP. Adoptive cell transfer as personalized immunotherapy for human cancer. Science (2015) 348:62-8. doi: 10.1126/science.aaa4967

132. Houot R, Schultz LM, Marabelle A, Kohrt H. T-cell-based immunotherapy: adoptive cell transfer and checkpoint inhibition. Cancer Immunol Res. (2015) 3:1115-22. doi: 10.1158/2326-6066.CIR-15-0190

133. Kochenderfer JN, Dudley ME, Kassim SH, Somerville RPT, Carpenter RO, Stetler-Stevenson M, et al. Chemotherapy-refractory diffuse large B-cell lymphoma and indolent B-cell malignancies can be effectively treated with autologous T cells expressing an anti-CD19 chimeric antigen receptor. J Clin Oncol. (2015) 33:540-9. doi: 10.1200/JCO.2014.56.2025
134. McKee S. Novartis' Kymriah First Cell Therapy to Win US Green Light. (2017) Available online at: http://www.pharmatimes.com/news/novartis_kymriah_ first_cell_therapy_to_win_us_green_light_1203820 (Accessed September 9, 2017).

135. Locke FL, Neelapu SS, Bartlett NL, Lekakis LJ, Miklos D, Jacobson CA, et al. Abstract CT019: Primary results from ZUMA-1: a pivotal trial of axicabtagene ciloleucel (axicel; KTE-C19) in patients with refractory aggressive non-Hodgkin lymphoma (NHL). Cancer Res. (2017) 77:CT019. doi: 10.1158/1538-7445.AM2017-CT019

136. Philippidis A. FDA Approves Gilead CAR-T Therapy Yescarta, Plans Regenerative Medicine Policy “Soon". Gen (2017) Available online at: https:// www.genengnews.com/gen-news-highlights/fda-approves-gilead-cart-therapy-yescarta-plans-regenerative-medicine-policy-soon/81255071 (Accessed October 21, 2017).

137. Berdeja JG, Lin Y, Raje N, Munshi N, Siegel D, Liedtke M, et al. Durable clinical responses in heavily pretreated patients with relapsed/refractory multiple myeloma: updated results from a multicenter study of bb2121 antiBcma CAR T cell therapy. In: 59th Annual Meeting \& Exposition. Atlanta (2017). Available online at: https://ash.confex.com/ash/2017/webprogram/ Paper107984.html (Accessed January 7, 2018).

138. Smith TT, Moffett HF, Stephan SB, Opel CF, Dumigan AG, Jiang X, et al. Biopolymers codelivering engineered $\mathrm{T}$ cells and STING agonists can eliminate heterogeneous tumors. J Clin Invest. (2017) 60:2176-91. doi: 10.1172/JCI87624

139. Olweus J. Manufacture of CAR-T cells in the body. Nat Biotechnol. (2017) 35:520-1. doi: 10.1038/nbt.3898

140. Smith TT, Stephan SB, Moffett HF, McKnight LE, Ji W, Reiman $\mathrm{D}$, et al. In situ programming of leukaemia-specific $\mathrm{T}$ cells using synthetic DNA nanocarriers. Nat Nanotechnol. (2017) 12:813-20. doi: 10.1038/nnano.2017.57

141. Mi Y, Smith C, Yang F, Wang A. Spatial-temporal delivery of OX40 agonist and PD-1 inhibitor using nanoparticles improves therapeutic efficacy of cancer immunotherapy. In: AACR Annual Meeting 2017. Washington, DC (2017). Available online at: http://www.abstractsonline.com/pp8/\#!/4292/ presentation/5334 (Accessed April 6, 2017).

142. Fiering S. Cancer immunotherapy: making allies of phagocytes. Nat Nanotechnol. (2017) 12:615-6. doi: 10.1038/nnano. 2017.89

143. Luo M, Wang H, Wang Z, Cai H, Lu Z, Li Y, et al. A STING-activating nanovaccine for cancer immunotherapy. Nat Nanotechnol. (2017) 12:648-54. doi: $10.1038 /$ nnano.2017.52

144. Marchini A, Scott E, Rommelaere J. Overcoming barriers in oncolytic virotherapy with HDAC inhibitors and immune checkpoint blockade. Viruses (2016) 8:9. doi: 10.3390/v8010009

145. Lichty BD, Breitbach CJ, Stojdl DF, Bell JC. Going viral with cancer immunotherapy. Nat Rev Cancer (2014) 14:559-67. doi: 10.1038/nrc3770

146. Andtbacka RHI, Kaufman HL, Collichio F, Amatruda T, Senzer N, Chesney J, et al. Talimogene laherparepvec improves durable response rate in patients with advanced melanoma. J Clin Oncol. (2015) 33:2780-8. doi: $10.1200 /$ JCO.2014.58.3377

147. Lawrence L. Immunotherapy combination promising in advanced melanoma. Oncol J. (2017). Available online at: http://www.cancernetwork. $\mathrm{com} / \mathrm{melanoma/immunotherapy-combination-promising-advanced-}$ melanoma (Accessed April 4, 2017)

148. Liu Z, Ravindranathan R, Kalinski P, Guo ZS, Bartlett DL. Rational combination of oncolytic vaccinia virus and PD-L1 blockade works synergistically to enhance therapeutic efficacy. Nat Commun. (2017) 8:14754. doi: 10.1038/ncomms14754

149. $\mathrm{Bu} \mathrm{X}$, Mahoney KM, Freeman GJ. Learning from PD-1 resistance: new combination strategies. Trends Mol Med. (2016) 22:448-51. doi: 10.1016/j.molmed.2016.04.008

150. Samson A, Scott KJ, Taggart D, West EJ, Wilson E, Nuovo GJ, et al. Intravenous delivery of oncolytic reovirus to brain tumor patients immunologically primes for subsequent checkpoint blockade. Sci Transl Med. (2018) 10:eaam7577. doi: 10.1126/scitranslmed.aam7577

151. Diggs LP, Hsueh EC. Utility of PD-L1 immunohistochemistry assays for predicting PD-1/PD-L1 inhibitor response. Biomark Res. (2017) 5:12. doi: $10.1186 /$ s40364-017-0093-8 
152. Nduom EK, Wei J, Yaghi NK, Huang N, Kong L-Y, Gabrusiewicz K, et al. PDL1 expression and prognostic impact in glioblastoma. Neuro Oncol. (2016) 18:195-205. doi: 10.1093/neuonc/nov172

153. Bourgeois-Daigneault M-C, Roy DG, Aitken AS, El Sayes N, Martin NT, Varette $\mathrm{O}$, et al. Neoadjuvant oncolytic virotherapy before surgery sensitizes triple-negative breast cancer to immune checkpoint therapy. Sci Transl Med. (2018) 10:eaao1641. doi: 10.1126/scitranslmed.aao1641

154. Zhu Z, Gorman MJ, McKenzie LD, Chai JN, Hubert CG, Prager BC, et al. Zika virus has oncolytic activity against glioblastoma stem cells. J Exp Med. (2017) 214:2843-57. doi: 10.1084/jem.20171093

155. Nissim L, Wu M-R, Pery E, Binder-Nissim A, Suzuki HI, Stupp D, et al. Synthetic RNA-based immunomodulatory gene circuits for cancer immunotherapy. Cell (2017) 171:1138-50.e15. doi: 10.1016/j.cell.2017.09.049

156. Missiaen R, Morales-Rodriguez F, Eelen G, Carmeliet P. Targeting endothelial metabolism for anti-angiogenesis therapy: A pharmacological perspective. Vascul Pharmacol. (2017) 90:8-18. doi: 10.1016/j.vph.2017.01.001

157. van Baren N, Van den Eynde BJ. Tryptophan-degrading enzymes in tumoral immune resistance. Front Immunol. (2015) 6:34. doi: 10.3389/fimmu.2015.00034

158. Flint TR, Janowitz T, Connell CM, Roberts EW, Denton AE, Coll AP, et al. Tumor-induced IL-6 reprograms host metabolism to suppress anti-tumor immunity. Cell Metab (2016) 24:672-84. doi: 10.1016/j.cmet.2016.10.010

159. Chang C-H, Qiu J, O’Sullivan D, Buck MD, Noguchi T, Curtis JD, et al. Metabolic competition in the tumor microenvironment is a driver of cancer progression. Cell (2015) 162:1229-41. doi: 10.1016/j.cell.2015.08.016

160. Moon YW, Hajjar J, Hwu P, Naing A. Targeting the indoleamine 2,3-dioxygenase pathway in cancer. J Immunother Cancer (2015) 3:51. doi: 10.1186/s40425-015-0094-9

161. Herranz D, Ambesi-Impiombato A, Sudderth J, Sánchez-Martín M, Belver $\mathrm{L}$, Tosello V, et al. Metabolic reprogramming induces resistance to antiNOTCH1 therapies in T cell acute lymphoblastic leukemia. Nat Med. (2015) 21:1182-9. doi: 10.1038/nm.3955

162. Zelenay S, van der Veen AG, Böttcher JP, Snelgrove KJ, Rogers N, Acton $\mathrm{SE}$, et al. Cyclooxygenase-dependent tumor growth through evasion of immunity. Cell (2015) 162:1257-70. doi: 10.1016/j.cell.2015.08.015

163. Kishore M, Cheung KCP, Fu H, Bonacina F, Wang G, Coe D, et al. Regulatory $\mathrm{T}$ cell migration is dependent on glucokinase-mediated glycolysis. Immunity (2017) 47:875-89.e10. doi: 10.1016/j.immuni.2017.10.017

164. Markosyan N, Chen EP, Smyth EM. Targeting COX-2 abrogates mammary tumorigenesis: breaking cancer-associated suppression of immunosurveillance. Oncoimmunology (2014) 3:e29287. doi: 10.4161/onci.29287

165. Naka K, Jomen Y, Ishihara K, Kim J, Ishimoto $\mathrm{T}$, Bae E-J, et al. Dipeptide species regulate p38MAPK-Smad3 signalling to maintain chronic myelogenous leukaemia stem cells. Nat Commun. (2015) 6:8039. doi: $10.1038 /$ ncomms 9039

166. Silver DJ, Sinyuk M, Vogelbaum MA, Ahluwalia MS, Lathia JD. The intersection of cancer, cancer stem cells, and the immune system: therapeutic opportunities. Neuro Oncol (2016) 18:153-9. doi: 10.1093/neuonc/nov157

167. Beger RD, Dunn W, Schmidt MA, Gross SS, Kirwan JA, Cascante M, et al. Metabolomics enables precision medicine: "A White Paper, Community Perspective”. Metabolomics (2016) 12:149. doi: 10.1007/s11306-016-1094-6

168. Zhang X, Yuan X, Shi H, Wu L, Qian H, Xu W. Exosomes in cancer: small particle, big player. J Hematol Oncol. (2015) 8:83. doi: 10.1186/s13045-015-0181-x

169. Bell BM, Kirk ID, Hiltbrunner S, Gabrielsson S, Bultema JJ. Designer exosomes as next-generation cancer immunotherapy. Nanomed Nanotechnol Biol Med. (2016) 12:163-9. doi: 10.1016/j.nano.2015.09.011

170. Romagnoli GG, Zelante BB, Toniolo PA, Migliori IK, Barbuto JAM. Dendritic cell-derived exosomes may be a tool for cancer immunotherapy by converting tumor cells into immunogenic targets. Front Immunol (2014) 5:692. doi: 10.3389/fimmu.2014.00692

171. Gehrmann U, Näslund TI, Hiltbrunner S, Larssen P. Harnessing the exosome-induced immune response for cancer immunotherapy. Semin Cancer Biol. (2014) 28:58-67. doi: 10.1016/j.semcancer.2014.05.003
172. Zhang X, Pei Z, Chen J, Ji C, Xu J, Zhang X, et al. Exosomes for immunoregulation and therapeutic intervention in cancer. J Cancer (2016) 7:1081-7. doi: 10.7150/jca.14866

173. Kim MS, Haney MJ, Zhao Y, Mahajan V, Deygen I, Klyachko NL, et al. Development of exosome-encapsulated paclitaxel to overcome MDR in cancer cells. Nanomed Nanotechnol Biol Med. (2016) 12:655-64. doi: 10.1016/j.nano.2015.10.012

174. Yoshimura A, Sawada K, Kimura T. Is the exosome a potential target for cancer immunotherapy? Ann Transl Med. (2017) 5:117. doi: 10.21037/atm.2017.01.47

175. Li Q, Huang Q, Huyan T, Wang Y, Huang Q, Shi J. Bifacial effects of engineering tumour cell-derived exosomes on human natural killer cells. Exp Cell Res. (2018) 363:141-50. doi: 10.1016/J.YEXCR.2017.12.005

176. Pollizzi KN, Sun I-H, Patel CH, Lo Y-C, Oh M-H, Waickman AT, et al. Asymmetric inheritance of mTORC1 kinase activity during division dictates CD8+ T cell differentiation. Nat Immunol. (2016) 17:704-11. doi: $10.1038 /$ ni.3438

177. Widjaja CE, Olvera JG, Metz PJ, Phan AT, Savas JN, de Bruin G, et al. Proteasome activity regulates CD8 $+\mathrm{T}$ lymphocyte metabolism and fate specification. J Clin Invest. (2017) 127:3609-23. doi: 10.1172/JCI90895

178. Feske S, Wulff H, Skolnik EY. Ion channels in innate and adaptive immunity. Annu Rev Immunol. (2015) 33:291-353. doi: 10.1146/annurev-immunol-032414-112212

179. Lo W-L, Donermeyer DL, Allen PM. A voltage-gated sodium channel is essential for the positive selection of CD4+ T cells. Nat Immunol. (2012) 13:880-7. doi: 10.1038/ni.2379

180. Malissen B. A voltage-gated sodium channel mediates positive selection of T cells. Nat Immunol. (2012) 13:810-2. doi: 10.1038/ni.2400

181. Seo AN, Lee HJ, Kim EJ, Kim HJ, Jang MH, Lee HE, et al. Tumour-infiltrating CD8+ lymphocytes as an independent predictive factor for pathological complete response to primary systemic therapy in breast cancer. Br J Cancer (2013) 109:2705-13. doi: 10.1038/bjc.2013.634

182. Jing W, Gershan JA, Johnson BD. Depletion of CD4 T cells enhances immunotherapy for neuroblastoma after syngeneic HSCT but compromises development of antitumor immune memory. Blood (2009) 113:4449-57. doi: 10.1182/blood-2008-11-190827

183. Roh-Johnson M, Shah AN, Stonick JA, Poudel KR, Kargl J, Yang $\mathrm{GH}$, et al. Macrophage-dependent cytoplasmic transfer during melanoma invasion in vivo. Dev Cell (2017) 43:549-62.e6. doi: 10.1016/j.devcel.2017. 11.003

184. Black JA, Waxman SG. Noncanonical roles of voltage-gated sodium channels. Neuron (2013) 80:280-91. doi: 10.1016/j.neuron.2013.09.012

185. Fraser SP, Diss JKJ, Chioni A-M, Mycielska ME, Pan H, Yamaci RF, et al. Voltage-gated sodium channel expression and potentiation of human breast cancer metastasis. Clin Cancer Res. (2005) 11:5381-9. doi: 10.1158/1078-0432.CCR-05-0327

186. Baptista-Hon DT, Robertson FM, Robertson GB, Owen SJ, Rogers GW, Lydon EL, et al. Potent inhibition by ropivacaine of metastatic colon cancer SW620 cell invasion and Na V 1.5 channel function. Br J Anaesth. (2014) 113:i39-48. doi: 10.1093/bja/aeu104

187. Chioni A-M, Fraser SP, Pani F, Foran P, Wilkin GP, Diss JKJ, et al. A novel polyclonal antibody specific for the Nav1.5 voltage-gated $\mathrm{Na}+$ channel 'neonatal' splice form. J Neurosci Methods (2005) 147:88-98. doi: 10.1016/j.jneumeth.2005.03.010

188. Yamaci RF, Fraser SP, Battaloglu E, Kaya H, Erguler K, Foster CS, et al. Neonatal Nav1.5 protein expression in normal adult human tissues and breast cancer. Pathol Res Pract. (2017) 213:900-7. doi: 10.1016/j.prp.2017.06.003

189. Fairhurst C, Watt I, Martin F, Bland M, Brackenbury WJ. Sodium channelinhibiting drugs and survival of breast, colon and prostate cancer: a population-based study. Sci Rep. (2015) 5:16758. doi: 10.1038/srep16758

190. Swann JB, Smyth MJ. Immune surveillance of tumors. J Clin Invest. (2007) 117:1137-46. doi: 10.1172/JCI31405

191. Serrano-Albarrás A, Estadella I, Cirera-Rocosa S, NavarroPérez M, Felipe A. Kv1.3: a multifunctional channel with many pathological implications. Expert Opin Ther Targets (2018) 22:101-5. doi: 10.1080/14728222.2017.1420170 
192. Chimote AA, Hajdu P, Sfyris AM, Gleich BN, Wise-Draper T, Casper KA, Conforti L. Kv1.3 channels mark functionally competent CD8 ${ }^{+}$tumorinfiltrating lymphocytes in head and neck cancer. Cancer Res. (2017) 77:5361. doi: 10.1158/0008-5472.CAN-16-2372

193. Panyi G, Beeton C, Felipe A. Ion channels and anti-cancer immunity. Philos Trans R Soc Lond B Biol Sci. (2014) 369:20130106. doi: $10.1098 /$ rstb.2013.0106

194. Eil R, Vodnala SK, Clever D, Klebanoff CA, Sukumar M, Pan JH, et al. Ionic immune suppression within the tumour microenvironment limits T cell effector function. Nature (2016) 537:539-43. doi: 10.1038/nature 19364

195. Liu Y, Zhao L, Ma W, Cao X, Chen H, Feng D, et al. The blockage of KCa3.1 channel inhibited proliferation, migration and promoted apoptosis of human hepatocellular carcinoma cells. J Cancer (2015) 6:643-51. doi: 10.7150/jca.11913

196. Koshy S, Wu D, Hu X, Tajhya RB, Huq R, Khan FS, et al. Blocking KCa3.1 channels increases tumor cell killing by a subpopulation of human natural killer lymphocytes. PLoS ONE (2013) 8:e76740. doi: 10.1371/journal.pone.0076740

197. MacFarlane AW, Jillab M, Plimack ER, Hudes GR, Uzzo RG, Litwin S, et al. PD-1 expression on peripheral blood cells increases with stage in renal cell carcinoma patients and is rapidly reduced after surgical tumor resection. Cancer Immunol Res. (2014) 2:320-31. doi: 10.1158/2326-6066.CIR-13-0133

198. Klingemann H, Boissel L, Toneguzzo F. Natural killer cells for immunotherapy - advantages of the NK-92 cell line over blood NK cells. Front Immunol. (2016) 7:91. doi: 10.3389/fimmu.2016.00091

199. Redmond J, O'Rilley D, Buchanan P. Role of ion channels in natural killer cell function towards cancer. Discov Med. (2017) 23:353-360.

200. Bose T, Cieślar-Pobuda A, Wiechec E. Role of ion channels in regulating $\mathrm{Ca}^{2+}$ homeostasis during the interplay between immune and cancer cells. Cell Death Dis (2015) 6:e1648. doi: 10.1038/cddis.2015.23

201. Litan A, Langhans SA. Cancer as a channelopathy: ion channels and pumps in tumor development and progression. Front Cell Neurosci. (2015) 9:86. doi: 10.3389/fncel.2015.00086

202. Tan P, He L, Han G, Zhou Y. Optogenetic immunomodulation: shedding light on antitumor immunity. Trends Biotechnol. (2017) 35:215-26. doi: 10.1016/j.tibtech.2016.09.002

203. Topalian SL, Taube JM, Anders RA, Pardoll DM. Mechanism-driven biomarkers to guide immune checkpoint blockade in cancer therapy. Nat Rev Cancer (2016) 16:275-87. doi: 10.1038/nrc.2016.36

204. Teng MWL, Ngiow SF, Ribas A, Smyth MJ. Classifying cancers based on T-cell infiltration and PD-L1. Cancer Res. (2015) 75:2139-45. doi: 10.1158/0008-5472.CAN-15-0255

205. Danilova L, Wang H, Sunshine J, Kaunitz GJ, Cottrell TR, Xu H, et al. Association of PD-1/PD-L axis expression with cytolytic activity, mutational load, and prognosis in melanoma and other solid tumors. Proc Natl Acad Sci USA. (2016) 113:E7769-77. doi: 10.1073/pnas.1607836113

206. Snyder A, Makarov V, Merghoub T, Yuan J, Zaretsky JM, Desrichard A, et al. Genetic basis for clinical response to CTLA-4 blockade in melanoma. N Engl J Med. (2014) 371:2189-99. doi: 10.1056/NEJMoa1406498

207. Miller L. Mutational load is only one piece of the immunotherapy puzzle. Target Oncol. (2017). Available online at: https://www.targetedonc.com/ publications/targeted-therapy-news/2017/february-2017/mutational-loadis-only-one-piece-of-the-immunotherapy-puzzle (Accessed March 22, 2017)

208. Frampton GM, Fabrizio DA, Chalmers ZR, Sun JX, Miller VA, Stephens PJ. Assessment and comparison of tumor mutational burden and microsatellite instability status in \&gt;40,000 cancer genomes. Ann Oncol. (2016) 27:15-42. doi: 10.1093/annonc/mdw363.01

209. Tanase CP, Codrici E, Popescu ID, Mihai S, Enciu A-M, Necula LG, et al. Prostate cancer proteomics: Current trends and future perspectives for biomarker discovery. Oncotarget (2017) 8:18497-512. doi: 10.18632/oncotarget.14501

210. Mehnert JM, Monjazeb AM, Beerthuijzen JMT, Collyar D, Rubinstein L, Harris LN. The challenge for development of valuable immuno-oncology biomarkers. Clin Cancer Res (2017) 23:4970-9. doi: 10.1158/1078-0432.CCR-16-3063
211. Anagnostou V, Yarchoan M, Hansen AR, Wang H, Verde F, Sharon E, et al. Immuno-oncology trial endpoints: capturing clinically meaningful activity. Clin Cancer Res (2017 23:4959-69. doi: 10.1158/1078-0432.CCR-16-3065

212. Wilson MK, Karakasis K, Oza AM. Outcomes and endpoints in trials of cancer treatment: the past, present, and future. Lancet Oncol. (2015) 16:e3242. doi: 10.1016/S1470-2045(14)70375-4

213. Lesterhuis WJ, Bosco A, Millward MJ, Small M, Nowak AK, Lake RA. Dynamic versus static biomarkers in cancer immune checkpoint blockade: unravelling complexity. Nat Rev Drug Discov. (2017) 16:264-72. doi: $10.1038 / \mathrm{nrd} .2016 .233$

214. Vonderheide RH, Nathanson KL. The road to personalized cancer vaccines. Nat Med. (2013) 19:1098-100. doi: 10.1038/nm.3317y

215. Fridman WH, Zitvogel L, Sautès-Fridman C, Kroemer G. The immune contexture in cancer prognosis and treatment. Nat Rev Clin Oncol. (2017) 14:717-34. doi: 10.1038/nrclinonc.2017.101

216. Krieg C, Nowicka M, Guglietta S, Schindler S, Hartmann FJ, Weber LM, et al. High-dimensional single-cell analysis predicts response to anti-PD-1 immunotherapy. Nat Med. (2018) 24:144-53. doi: 10.1038/nm.4466

217. Tunstall KL. Quantifying immune cell distribution in the tumor microenvironment using HALO TM spatial analysis tools. (2016) Available online at: https://thepathologist.com/fileadmin/issues/App_Notes/0016-010-haloapp-note.pdf (Accessed July, 2016).

218. Au Q, Nguyen K, Padmanabhan R, Kuller A, Moler E, Hoe N. Abstract 4146: MultiOmyx multiplexed tumor infiltrating lymphocyte panel provides comprehensive immunophenotyping from a single FFPE slide. Cancer Res. (2016) 76. 10.1158/1538-7445.AM201 6-4146

219. Webb S. The cancer bloodhounds. Nat Biotechnol. (2016) 34:1090-4. doi: $10.1038 /$ nbt.3717

220. Zill O, Mortimer S, Banks K, Nagy R, Chudova D. Somatic genomic landscape of over 15,000 patients with advanced-stage cancer from clinical next-generation sequencing analysis of circulating tumor DNA. In: 2016 ASCO Annual Meeting | Abstracts | Meeting Library. Chicago, IL: Journal of Clinical Oncology. doi: 10.1200/JCO.2016.34.18_suppl.LBA11501 (Accessed April 12, 2017).

221. Han X, Wang J, Sun Y. Circulating tumor DNA as biomarkers for cancer detection. Genom Proteom Bioinform. (2017) 15:59-72. doi: 10.1016/j.gpb.2016.12.004

222. Siravegna G, Marsoni S, Siena S, Bardelli A. Integrating liquid biopsies into the management of cancer. Nat Rev Clin Oncol. (2017) 14:531-48. doi: $10.1038 /$ nrclinonc. 2017.14

223. Connelly S. Liquid biopsies may never replace tissue biopsies, Wistuba says. Target Oncol. (2017). Available online at: https://www.targetedonc.com/ publications/targeted-therapy-news/2017/august-2017/liquid-biopsiesmay-never-replace-tissue-biopsies-wistuba-says (Accessed August 14, 2017)

224. Schumacher TN, Schreiber RD. Neoantigens in cancer immunotherapy. Science (2015) 348:69-74. doi: 10.1126/science.aaa4971

225. Kvistborg P, Clynes R, Song W, Yuan J. Immune monitoring technology primer: whole exome sequencing for neoantigen discovery and precision oncology. J Immunother Cancer (2016) 4:22. doi: 10.1186/s40425-016-0126-0

226. Porta-Pardo E, Godzik A. Mutation drivers of immunological responses to cancer. Cancer Immunol Res. (2016) 4:789-8. doi: 10.1158/2326-6066.CIR-15-0233

227. Kataoka K, Shiraishi Y, Takeda Y, Sakata S, Matsumoto M, Nagano S, et al. Aberrant PD-L1 expression through 3 '-UTR disruption in multiple cancers. Nature (2016) 534:402-6. doi: 10.1038/nature18294

228. Zhang W, Bojorquez-Gomez A, Velez DO, Xu G, Sanchez KS, Shen JP, et al. A global transcriptional network connecting noncoding mutations to changes in tumor gene expression. Nat Genet. (2018) 50:613-20. doi: 10.1038/s41588-018-0091-2

229. Thorsson V, Gibbs DL, Brown SD, Wolf D, Bortone DS, Ou Yang T-H, et al. The immune landscape of cancer. Immunity (2018) 48:812-30.e14. doi: 10.1016/j.immuni.2018.03.023

230. Pompili L, Porru M, Caruso C, Biroccio A, Leonetti C. Patient-derived xenografts: a relevant preclinical model for drug development. J Exp Clin Cancer Res. (2016) 35:189. doi: 10.1186/s13046-016-0462-4 
231. Schook LB, Collares TV, Hu W, Liang Y, Rodrigues FM, Rund LA, et al. A genetic porcine model of cancer. PLoS ONE (2015) 10:e0128864. doi: 10.1371/journal.pone.0128864

232. Sun R, Johanna Limkin E, Dercle L. A non-invasive computational imaging approach may help predict response to immunotherapy. In: AACR-NCI-EORTC International Conference on Molecular Targets and Cancer Therapeutics. Available online at: http://mb.cision.com/Public/3069/ 2371973/9b82aa9989336285.pdf (Accessed November 12, 2017).

233. Garber K. Oncologists await historic first: a pan-tumor predictive marker, for immunotherapy. Nat Biotechnol. (2017) 35:297-8. doi: 10.1038/nbt0417-297a

234. Phan-Lai V, Florczyk SJ, Kievit FM, Wang K, Gad E, Disis ML, et al. Threedimensional scaffolds to evaluate tumor associated fibroblast-mediated suppression of breast tumor specific T cells. Biomacromolecules (2013) 14:1330-7. doi: 10.1021/bm301928u

235. Zanoni M, Piccinini F, Arienti C, Zamagni A, Santi S, Polico R, et al. $3 \mathrm{D}$ tumor spheroid models for in vitro therapeutic screening: a systematic approach to enhance the biological relevance of data obtained. Sci Rep. (2016) 6:19103. doi: 10.1038/srep19103

236. Devaud C, Westwood JA, Teng MW, John LB, Yong CS, Duong CP, et al. Differential potency of regulatory $\mathrm{T}$ cell-mediated immunosuppression in kidney tumors compared to subcutaneous tumors. Oncoimmunology (2014) 3:e963395. doi: 10.4161/21624011.2014.963395

237. Mitchell MJ, Jain RK, Langer R. Engineering and physical sciences in oncology: challenges and opportunities. Nat Rev Cancer (2017) 17:659-75. doi: $10.1038 /$ nrc. 2017.83

238. Mills CD, Lenz LL, Harris RA. A breakthrough: macrophagedirected cancer immunotherapy. Cancer Res (2016) 76:513-6. doi: 10.1158/0008-5472.CAN-15-1737

239. El Osta B, Hu F, Sadek R, Chintalapally R, Tang S-C. Not all immunecheckpoint inhibitors are created equal: Meta-analysis and systematic review of immune-related adverse events in cancer trials. Crit Rev Oncol Hematol. (2017) 119:1-12. doi: 10.1016/j.critrevonc.2017.09.002

240. Tabassum DP, Polyak K. Tumorigenesis: it takes a village. (2015) 15:473-83. doi: $10.1038 / \mathrm{nrc} 3971$

241. Luskin MR, Murakami MA, Manalis SR, Weinstock DM. Targeting minimal residual disease: a path to cure? Nat Rev Cancer (2018) 18:255-63. doi: $10.1038 /$ nrc. 2017.125

242. Roy S, Trinchieri G. Microbiota: a key orchestrator of cancer therapy. Nat Rev Cancer (2017) 17:271-85. doi: 10.1038/nrc.2017.13

243. Sivan A, Corrales L, Hubert N, Williams JB, Aquino-Michaels K, Earley ZM, et al. Commensal Bifidobacterium promotes antitumor immunity and facilitates anti-PD-L1 efficacy. Science (2015) 350:1084-9. doi: 10.1126/science.aac4255

244. Matson V, Fessler J, Bao R, Chongsuwat T, Zha Y, Alegre M-L, et al. The commensal microbiome is associated with anti-PD-1 efficacy in metastatic melanoma patients. Science (2018) 359:104-8. doi: 10.1126/SCIENCE.AAO3290

245. Koh A, Frankel A, Coughlin L. Metagenomic shotgun sequencing and unbiased metabolomic profiling identify specific human gut microbiota and metabolites associated with immune checkpoint therapy efficacy in melanoma patients. Neoplasia (2017) 19:848-55. doi: 10.1016/J.NEO.2017.08.004

246. Routy B, Le Chatelier E, Derosa L, Duong CPM, Alou MT, Daillère R, et al. Gut microbiome influences efficacy of PD-1-based immunotherapy against epithelial tumors. Science (2017) 359:91-7. doi: 10.1126/science. aan3706

247. Wang C, Sun W, Ye Y, Hu Q, Bomba HN, Gu Z, et al. In situ activation of platelets with checkpoint inhibitors for post-surgical cancer immunotherapy. Nat Biomed Eng. (2017) 1:0011. doi: 10.1038/s41551-016-0011

248. Ott PA, Hodi FS, Kaufman HL, Wigginton JM, Wolchok JD. Combination immunotherapy: a road map. J Immunother Cancer (2017) 5:16. doi: 10.1186/s40425-017-0218-5

249. Grinberg-Bleyer Y, Oh H, Desrichard A, Bhatt DM, Caron R, et al. NF-кB c-Rel is crucial for the regulatory $\mathrm{T}$ cell immune checkpoint in cancer. Cell (2017) 170:1096-108.e13. doi: 10.1016/j.cell.2017.08.004

250. Gong C, Ni Z, Yao C, Zhu X, Ni L, Wang L, et al. A high-throughput assay for screening of natural products that enhanced tumoricidal activity of NK cells. Biol Proced Online (2015) 17:12. doi: 10.1186/s12575-015-0 026-6

251. Ryall KA, Choon Tan A. Systems biology approaches for advancing the discovery of effective drug combinations. J Cheminform. (2015) 7:7. doi: 10.1186/s13321-015-0055-9

252. Doerstling SS, O'Flanagan CH, Hursting SD. Obesity and cancer metabolism: a perspective on interacting tumor-intrinsic and extrinsic factors. Front Oncol. (2017) 7:216. doi: 10.3389/fonc.2017.00216

253. Pantziarka P, Sukhatme V, Bouche G, Melhuis L, Sukhatme VP. Repurposing drugs in oncology (ReDO)-diclofenac as an anti-cancer agent. Ecancermedicalscience (2016) 10:610.doi: 10.3332/ecancer.20 16.610

254. McIntyre P. Accelerating Progress in Immunotherapy. Cancerworld (2016) Available at: http://cancerworld.net/cutting-edge/accelerating-progress-inimmunotherapy/ (Accessed July 12, 2016).

255. Ott PA, Hodi FS, Robert C. CTLA-4 and PD-1/PD-L1 blockade: new immunotherapeutic modalities with durable clinical benefit in melanoma patients. Clin Cancer Res. (2013) 19:5300-9. doi: 10.1158/1078-0432.CCR-13-0143

256. Su S, Hu B, Shao J, Shen B, Du J, Du Y, Zhou J, Yu L, Zhang L, Chen F, et al. CRISPR-Cas9 mediated efficient PD-1 disruption on human primary T cells from cancer patients. Sci Rep (2016) 6:20070. doi: 10.1038/srep20070

257. Carlsten M, Levy E, Karambelkar A, Li L, Reger R, Berg M, et al. Efficient mRNA-based genetic engineering of human NK cells with highaffinity CD16 and CCR7 augments rituximab-induced ADCC against lymphoma and targets NK cell migration toward the lymph node-associated chemokine CCL19. Front Immunol. (2016) 7:105. doi: 10.3389/fimmu.2016. 00105

258. Moffett HF, Coon ME, Radtke S, Stephan SB, McKnight L, Lambert A, et al. Hit-and-run programming of therapeutic cytoreagents using mRNA nanocarriers. Nat Commun. (2017) 8:389. doi: 10.1038/s41467-017-00505-8

259. Patel SJ, Sanjana NE, Kishton RJ, Eidizadeh A, Vodnala SK, Cam M, et al. Identification of essential genes for cancer immunotherapy. Nature (2017) 548:537-42. doi: 10.1038/nature23477

260. Dubrow A. Cancer Research: A Supercomputing Perspective - Latest News - Texas Advanced Computing Center. Texas Advanced Computing Center (2017) Available online at: https://www.tacc.utexas.edu/-/cancer-researcha-supercomputing-perspective (Accessed November 12, 2017).

261. Castelvecchi D. Quantum computers ready to leap out of the lab in 2017. Nat News (2017) 541:9. doi: 10.1038/541009a

262. Boutros C, Tarhini A, Routier E, Lambotte O, Leroy Ladurie F, Carbonnel F, et al. Safety profiles of anti-CTLA-4 and anti-PD-1 antibodies alone and in combination. Nat Rev Clin Oncol. (2016) 13:473-86. doi: $10.1038 /$ nrclinonc. 2016.58

263. McKee S. FDA Holds Trials of Cellectis' Cell Therapy After Patient Death. (2017) Available online at: http://www.pharmatimes.com/news/fda_holds trials_of_cellectis_cell_therapy_after_patient_death_1204313 (Accessed September 7, 2017).

264. Underwood G. FDA puts Bristol-Myers Squibb Opdivo Trials on Hold. (2017) Available online at: http://www.pharmatimes.com/news/fda_puts_bristolmyers_squibb_opdivo_trials_on_hols_1204534 (Accessed September 7, 2017).

Conflict of Interest Statement: MD is involved in a small biotechnology company aiming to exploit the anti-cancer potential of VGSCs.

The remaining author declares that the research was conducted in the absence of any commercial or financial relationships that could be construed as a potential conflict of interest.

The reviewer PL and handling Editor declared their shared affiliation.

Copyright (C) 2018 Marshall and Djamgoz. This is an open-access article distributed under the terms of the Creative Commons Attribution License (CC BY). The use, distribution or reproduction in other forums is permitted, provided the original author(s) and the copyright owner(s) are credited and that the original publication in this journal is cited, in accordance with accepted academic practice. No use, distribution or reproduction is permitted which does not comply with these terms. 